\title{
Cliff Feature Delineation Tool and Baseline Builder Tool, Version 1.0 User Guide
}

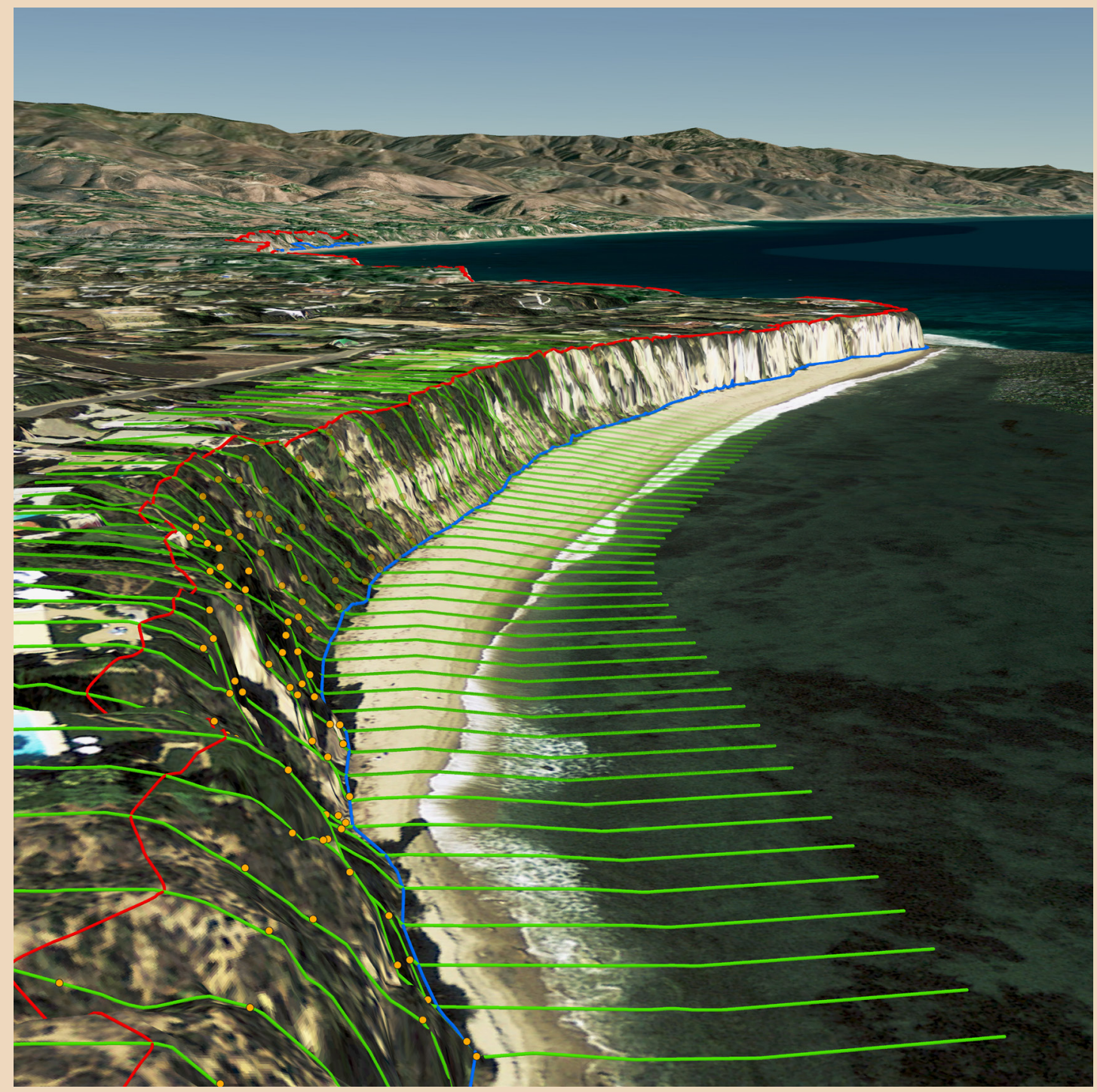

Open-File Report 2020-1070

U.S. Department of the Interior

U.S. Geological Survey 
Cover: Digital three-dimensional rendering of the coastal cliffs around Point Dume in Malibu, California. Cliff feature outputs from the Cliff Feature Delineation Tool are shown to conceptually display the cliff delineation process, including cross-shore transects (green), the cliff top line (red), the cliff toe line (blue), and subsidiary cliff features (orange). Base-map data credits: USGS, National Agriculture Imagery Program, NOAA, ESRI, DigitalGlobe, GeoEye, Earthstar Geographics, CNES/ Airbus DS, USDA, AeroGRID, IGN, and the GIS User Community. 


\section{Cliff Feature Delineation Tool and Baseline Builder Tool, Version 1.0 User Guide}

By Alexander C. Seymour, Cheryl J. Hapke, and Jonathan Warrick

Open-File Report 2020-1070

U.S. Department of the Interior

U.S. Geological Survey 


\title{
U.S. Department of the Interior DAVID BERNHARDT, Secretary
}

\author{
U.S. Geological Survey \\ James F. Reilly, Director
}

U.S. Geological Survey, Reston, Virginia: 2020

For more information on the USGS—-the Federal source for science about the Earth, its natural and living resources, natural hazards, and the environment-visit https://www.usgs.gov/ or call 1-888-ASK-USGS (1-888-275-8747).

For an overview of USGS information products, including maps, imagery, and publications, visit https://www.usgs.gov/pubprod/.

Any use of trade, firm, or product names is for descriptive purposes only and does not imply endorsement by the U.S. Government.

Although this information product, for the most part, is in the public domain, it also may contain copyrighted materials as noted in the text. Permission to reproduce copyrighted items must be secured from the copyright owner.

Suggested citation:

Seymour, A.C., Hapke, C.J., and Warrick, J., 2020, Cliff Feature Delineation Tool and Baseline Builder version 1.0 user guide: U.S. Geological Survey Open File Report 2020-1070, 54 p., https://doi.org/10.3133/ofr20201070.

Associated software release for this publication:

Seymour, A.C., Hapke, C.J., and Warrick, J., 2020, Cliff Feature Delineation Tool and Baseline Builder version 1.0: U.S. Geological Survey software release, https://doi.org/10.5066/P9UKW7PO.

ISSN 2331-1258 (online) 


\section{Contents}

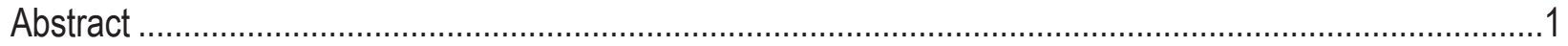

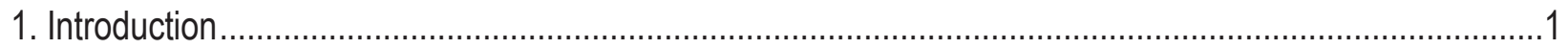

1.1 Defining the Cliff Feature Delineation Tool..................................................................................

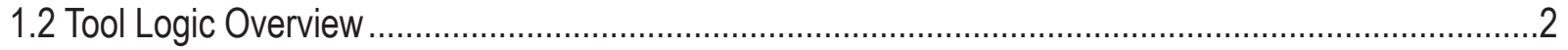

1.3 Cliff Feature Delineation at the U.S. Geological Survey .................................................................

1.4 Experimental Software Use Considerations................................................................................

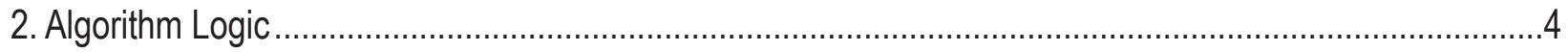

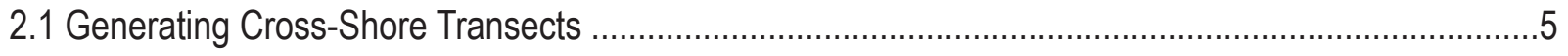

2.2 Extracting Surface Information Across Cross-Shore Transects ......................................................

2.3 Locating Cliff Tops, Midpoints, and Basic Toes .........................................................................

2.4 Refining the Cliff Toe Location ...........................................................................................

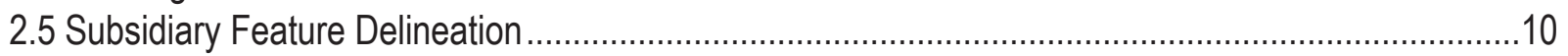

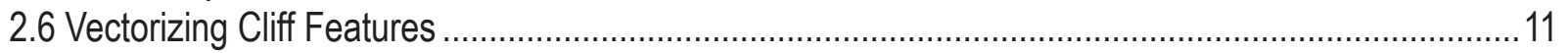

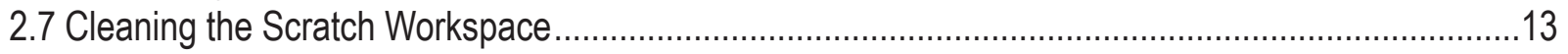

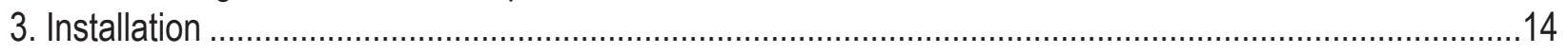

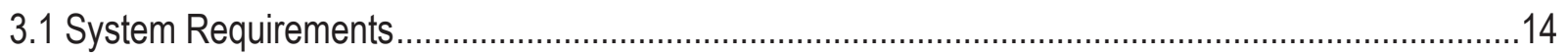

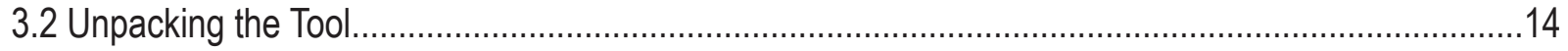

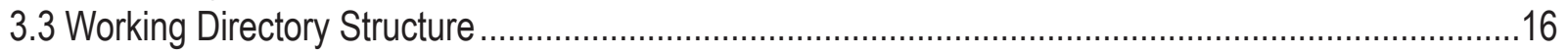

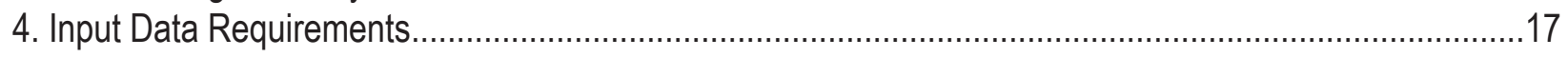

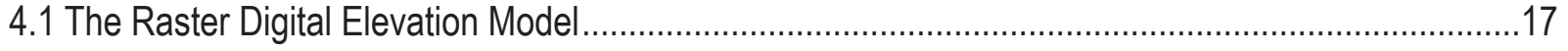

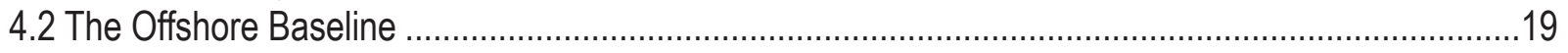

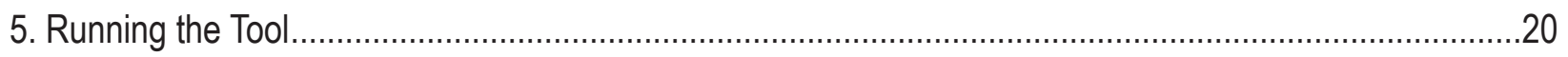

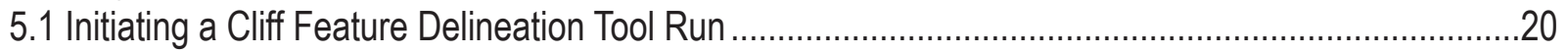

5.2 Explanation of Tool Inputs and Starting Parameters ................................................................21

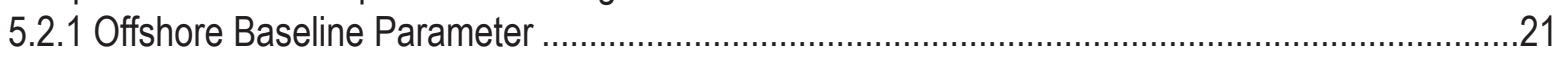

5.2.2 Smooth Baseline Parameter.................................................................................................

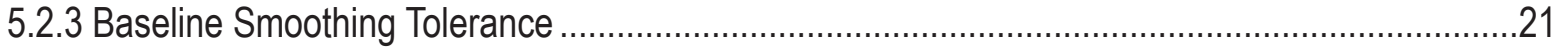

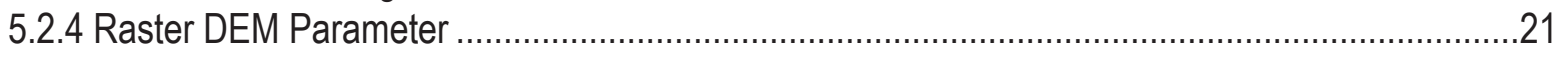

5.2.5 Landward Distance and Seaward Distance Parameters .......................................................21

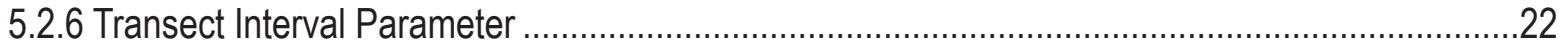

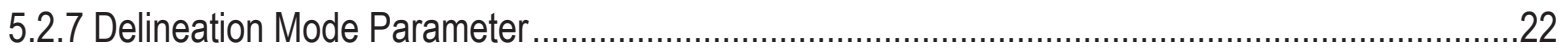

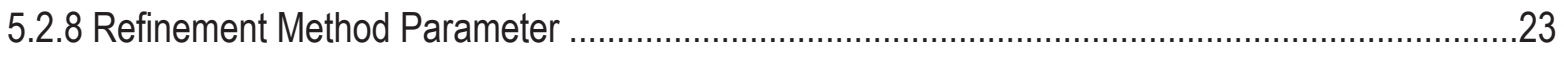

5.2.9 High Pass Threshold Parameter..........................................................................................2

5.2.10 Subsidiary Feature Delineation Parameter ..........................................................................23

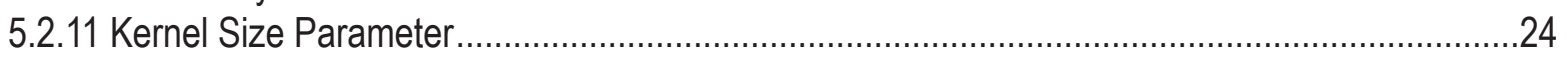

5.2.12 Output Smoothing Tolerance Parameter …………….........................................................

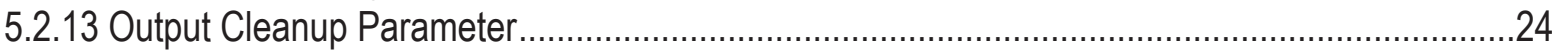

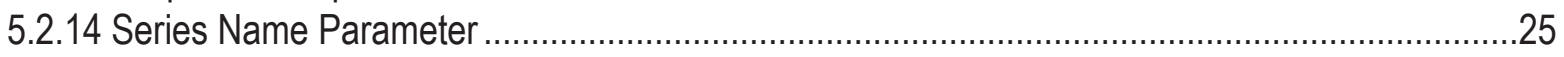

6. Using the Baseline Builder Tool and Vectorizing an Offshore Baseline ................................................26

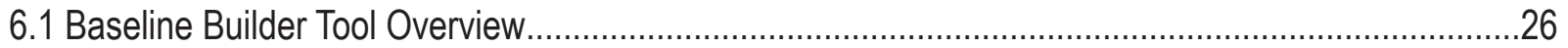

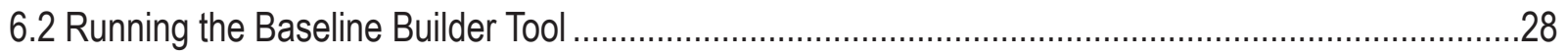

6.3 Explanation of Baseline Builder Tool Starting Parameters...........................................................2

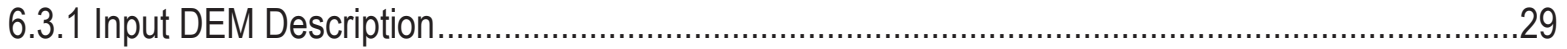




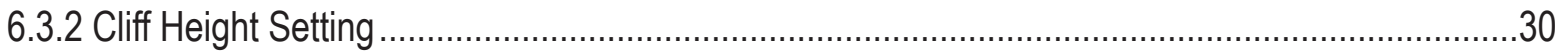

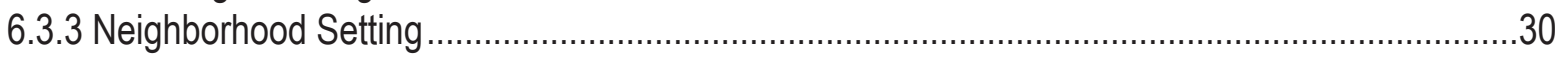

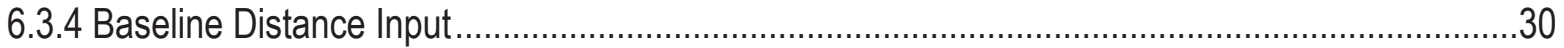

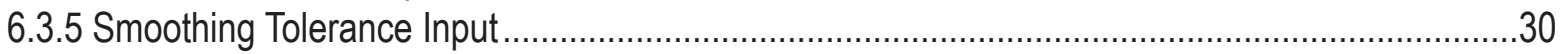

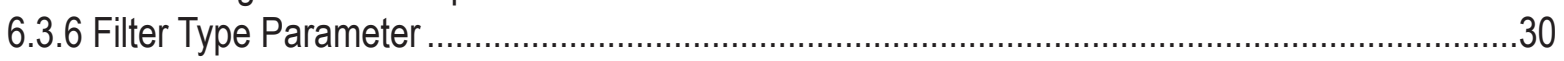

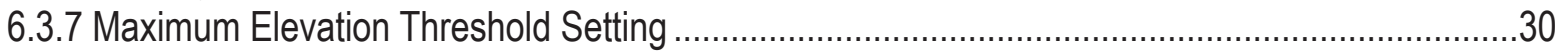

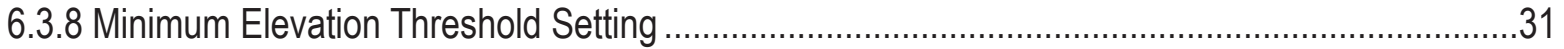

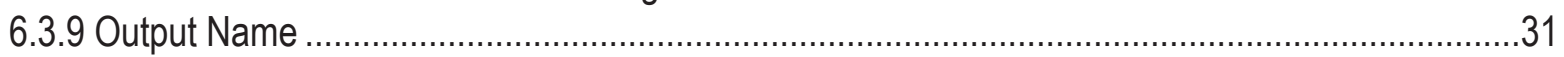

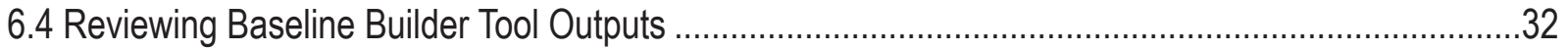

6.5 Manually Digitizing an Offshore Baseline...............................................................................

7. Visualizing and Reviewing Cliff Feature Delineation Tool Outputs .....................................................40

7.1 Visualizing Features in ArcScene..........................................................................................

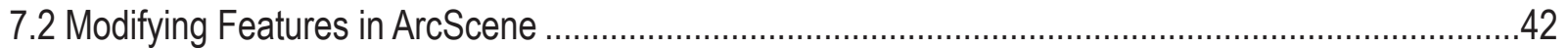

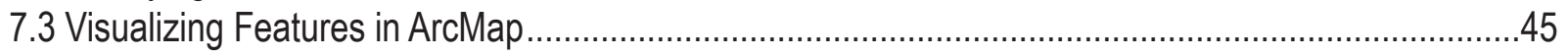

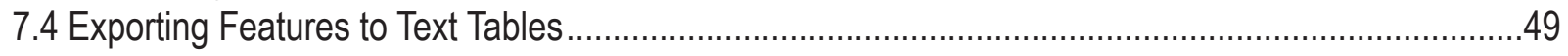

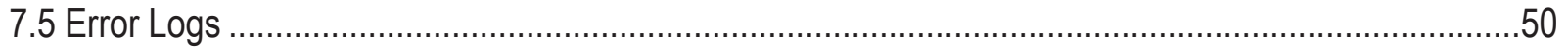

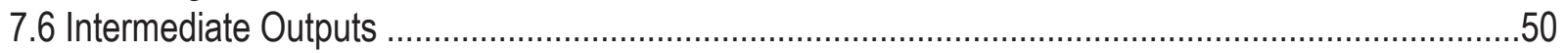

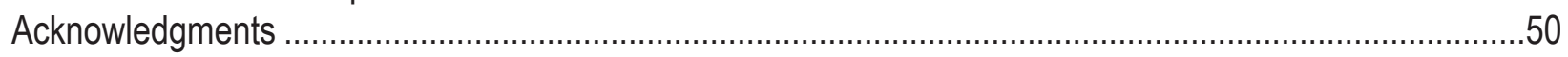

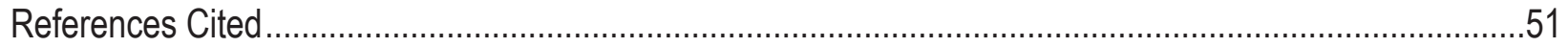

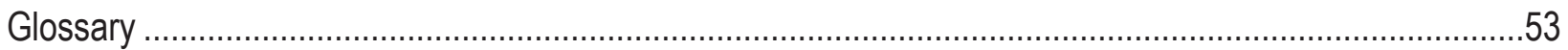

\section{Figures}

1. An elevation profile showing the upward sloping chord line projected across each transect............ 3

2. Maps showing the step-by-step process used by the algorithm to generate cross-shore transects . 6

3. Cliff elevation profiles showing corresponding high-pass profiles and surface plane intersections along a cross shore transect ................................................................................................ 8

4. A surface profile showing chord line length as a potential source of cliff feature misclassification ...9

5. Surface profiles showing examples of potential errors during high pass method toe refinement ... 10

6. A surface profile showing the possible outputs using the Cliff Feature Delineation Tool................. 11

7. Images showing selected outputs from the Cliff Feature Delineation Tool superimposed on a digital elevation model...................................................................................................... 12

8. An image showing all possible outputs of the Cliff Feature Delineation Tool shown for a short stretch of coast in the ArcScene 3D format......................................................................... 14

9. Instructions for setting up the Cliff Feature Delineation Tool and companion Baseline Builder

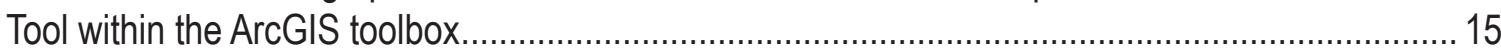

10. Images showing the processing steps taken by the Cliff Feature Delineation Tool to cast cross shore transects ........................................................................................................... 18

11. Instructions for initiating a Cliff Feature Delineation Tool run .................................................. 20

12. An image showing the cliff top line, cross-shore transects, and flagged cutback transects .............25

13. Images showing the processing steps taken by the Baseline Builder Tool to construct alongshore

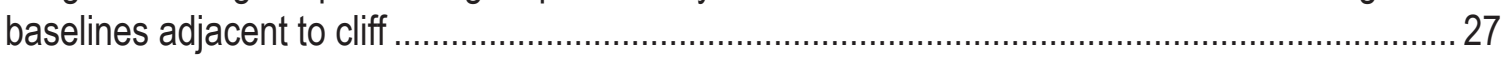

14. Instructions for running the Baseline Builder Tool ................................................................... 28

15. An image showing offshore baselines generated with different smoothing tolerances using the Baseline Builder Tool. 
16. Instructions for editing the Baseline Builder Tool output for use as an offshore baseline...............32

17. Instructions for manually digitizing a baseline, with the Baseline Builder Tool output used as a digitizing guide ..... 37

18. An image showing the ArcScene "Table of Contents" and "Symbol Selector," which can be used to change the symbology of output shapefiles

19. An image showing the key navigation tools for modifying the camera view in ArcScene, along with selected cliff feature outputs

20. Instructions for editing any cliff feature line in ArcScene ......................................................... 42

21. Instructions for graphing cross-shore transects and cliff feature points using ArcGIS Desktop ...... 46 


\section{Conversion Factors}

International System of Units to U.S. customary units

\begin{tabular}{|c|c|c|}
\hline Multiply & By & To obtain \\
\hline \multicolumn{3}{|c|}{ Length } \\
\hline meter (m) & 3.281 & foot $(\mathrm{ft})$ \\
\hline meter (m) & 1.094 & yard (yd) \\
\hline kilometer (km) & 0.6214 & mile (mi) \\
\hline kilometer $(\mathrm{km})$ & 0.5400 & mile, nautical (nmi) \\
\hline
\end{tabular}

\section{Datum}

Vertical coordinate information is referenced to the North American Vertical Datum of 1988 (NAVD 88).

\section{Abbreviations}

$\begin{array}{ll}\text { 2D } & \text { two dimensional } \\ \text { 3D } & \text { three-dimensional } \\ \text { CFDT } & \text { Cliff Feature Delineation Tool } \\ \text {.cSV } & \text { comma-separated values file } \\ \text { DEM } & \text { digital elevation model } \\ \text { DSAS } & \text { Digital Shoreline Analysis System } \\ \text { DSM } & \text { digital surface model } \\ \text {.geotiff } & \text { Georeferenced Tagged Image File Format } \\ \text { GIS } & \text { geographic information system } \\ \text { km } & \text { kilometer } \\ \text { m } & \text { meter } \\ \text { NAVD 88 } & \text { North American Vertical Datum of 1988 } \\ \text { sUAS } & \text { small unmanned aerial system } \\ \text { TIN } & \text { triangular irregular network } \\ \text {.txt } & \text { text file } \\ \text { USGS } & \text { U.S. Geological Survey } \\ \text { UTM } & \text { Universal Transverse Mercator } \\ \text {.zip } & \text { lossless data compression archive file }\end{array}$




\title{
Cliff Feature Delineation Tool and Baseline Builder Tool, Version 1.0 User Guide
}

\author{
By Alexander C. Seymour, Cheryl J. Hapke, and Jonathan Warrick
}

\begin{abstract}
Coastal cliffs constitute 80 percent of the world's coastline, with seacliffs fronting a large proportion of the U.S. West Coast shoreline, particularly in California. Erosion of coastal cliffs can threaten infrastructure and human life, yet the spatial and temporal scope of cliff studies have been limited by cumbersome traditional methods that rely on the manual interpretation of seacliff features - especially seacliff toes and top edges. The Cliff Feature Delineation Tool (CFDT) and the Baseline Builder Tool are designed to increase the efficiency of deriving seacliff features from remote sensing datasets by utilizing an automated, quantitative approach that eliminates traditional interpretive methods and ensures reproducibility. This document functions as a user guide for operating the Cliff Feature Delineation Tool and Baseline Builder Tool and includes a walkthrough of data-visualization and data-review workflows for the tools' three-dimensional (3D) cliff feature outputs. Also included is a brief overview of cliff feature delineation at the U.S. Geological Survey (USGS) and a detailed description of the tools' algorithmic logic.
\end{abstract}

\section{Introduction}

Coastal cliffs front approximately 80 percent of the world's shorelines (Emery and Kuhn, 1982), including long stretches of the U.S. coast where 1,300 kilometers ( $\mathrm{km})$ of seacliffs in California alone are in an erosive condition (Griggs and Patsch, 2004). Erosion in these areas threatens valuable real estate, undermines tourist economies (Hapke and Reid, 2007), and can directly endanger human life (Bird, 1994). Despite the widespread occurrence of seacliffs and coastal bluffs, the large spatial extent of coastal cliff coverage restricts the frequency of coastal change analysis by traditional, manual methods, which are time-consuming and have limited repeatability due to their reliance on human interpre tation of the imagery (Rutzinger and others, 2012; Ruggiero and others, 2013). Combined with the increasing availability of large scale, high-accuracy, high-resolution lidar and photogrammetric topographic datasets, seacliff change research can benefit greatly from automated image analysis and computer vision processes that map the morphological features of cliff environments. Outputs from automated algorithms can help validate predictive cliff-erosion models (Hapke and Plant, 2010) against a quantitative benchmark or help fill spatial and temporal data gaps that are increasingly constrained by manual analysis methods in an era of "big data." To this end, the Cliff Feature Delineation Tool (CFDT) and Baseline Builder Tool described in this report are designed to speed the spatial delineation of cliff morphology while removing the potential for variable human interpretation, ultimately making outputs more repeatable. 


\subsection{Defining the Cliff Feature Delineation Tool}

The CFDT is an automated computer-vision application designed to generate 3D line features representing the seacliff top, midpoint, and toe on high-relief coastal cliffs. The program also delineates 3D points on concavities and convexities across the cliff face (termed "subsidiary features"), which may represent talus deposits, secondary cliff tops, and secondary cliff toes. The tool package includes algorithms for automatically generating alongshore baselines (the Baseline Builder Tool), determining landward or seaward direction, and casting cross-shore transects with little or no user intervention. The application is freely available as a script tool for Esri ArcGIS Desktop version 10.6 and 10.7; generated 3D shapefiles can be reviewed in ArcGIS, converted to traditional cross-shore elevation profiles, exported as text tables, or manipulated in a 3D viewing environment such as Esri ArcScene. ${ }^{1}$ After cliff feature lines from two or more timepoints are delineated, line outputs can be combined with the Digital Shoreline Analysis System (DSAS; Himmelstoss, and others, 2018) to determine rates of cliff-top, midline, and toe migration in the same way that an analysis is conducted on a shoreline timeseries. This document serves as a guide for installing and operating the CFDT, as well as interpreting and conducting a data review on its outputs. Additionally, this guide includes a detailed description of CFDT algorithm logic and an overview of recent predecessors in the field of cliff feature delineation.

\subsection{Tool Logic Overview}

The CFDT uses two data inputs to generate cliff feature delineations: a raster digital elevation model ([DEM], derived from lidar or photogrammetry) and an offshore baseline automatically generated by the companion Baseline Builder Tool or digitized manually by the user. The core function of the CFDT is based on multiple cross-shore transects generated perpendicular to the offshore baseline input at a regular interval specified by the user. Cross-shore transects extract the DEM profile of the underlying cliff into a tabular format, where an algorithm casts a first-order polynomial "chord line" between the start and end of each cross-shore transect, representing an upward-sloping plane intersecting the cliff face (fig. 1) (Palaseanu-Lovejoy and others, 2016). Estimates of the cliff top, cliff toe, and cliff midpoint positions on each transect are determined by the maximum vertical distance between the plane and the profile surface. Toe position refinement can be conducted through two methods: value thresholding of a high-passfiltered slope raster derived from the input DEM or the filtering of convexities on the lower half of the cliff. Subsidiary features are flagged by casting additional chord lines on the upper and lower half of the cliff and executing additional concavity-convexity analysis. Top, toe, midline, and subsidiary feature estimates for each cross-shore transect are reprojected as shapefiles and converted into 3D line and point features using ArcGIS linear referencing.

As previously mentioned, the companion Baseline Builder Tool is responsible for automatically generating offshore baselines adjacent to the seacliffs represented in the DEM, satisfying a required input for the CFDT that would otherwise require manual digitization. This tool integrates user-specified height and area parameters into a spatial kernel, which scans the DEM

\footnotetext{
${ }^{1}$ Although the footnotes in this report link to information about tools in Esri ArcGIS Desktop version 10.6, information on the Esri ArcGIS Desktop version 10.7 tools can be found by following the links given in the footnotes of this report for the individual version 10.6 tools and selecting "10.7" on the "Other versions" dropdown list found on the page for each tool.
} 
for relief features. Once classified, the relief features are vectorized, buffered, smoothed, and then filtered by elevation statistics so that only line segments seaward of relief features near sea level are represented in the final baseline.

\subsection{Cliff Feature Delineation at the U.S. Geological Survey}

The CFDT was preceded by a workflow developed by Palaseanu-Lovejoy and others (2016) that was designed for seacliff feature delineation and bluff erosion analysis in the $\mathrm{R}$ programming environment. The workflow established the use of chord line projection across cross-shore transect elevation profiles, using the greatest chord line to elevation profile difference to determine cliff top and toe features. This process also included profile inflection analysis to identify cliffs as concave, convex, or complex. The Palaseanu-Lovejoy and others (2016) algorithm, along with subsequent modifications to the basic chord line method (Hapke and others, 2018), exist within a larger body of algorithm-based cliff delineation work that includes kernelbased DEM analysis (Richter and others, 2013), polynomial-based identification of surface-plane breakpoints (Briese, 2004), as well as image classification applied to satellite imagery (Di and others, 2003). In this context, the Palaseanu-Lovejoy and others (2016) algorithm provided a semiautomated solution for cliff feature delineation independent of surface slope, and it is, therefore, more robust when applied to varied or complex cliff morphologies.

Subsequent coastal-bluff erosion research within the USGS has been split between analyzing raw 3D point-cloud models (Warrick and others 2017; Warrick and others 2019) and improving upon the Palaseanu-Lovejoy and others (2016) algorithm for application with twodimensional (2D) DEMs (Hapke and others, 2018). Additions to the Palaseanu-Lovejoy and others (2016) technique have included cliff midpoint delineation to help discriminate between cliff changes driven by marine and terrestrial processes, along with kernel-based slope statistics and secondary chord lines (Hapke and others, 2018) to refine candidates for cliff top and toe locations. These secondary delineations may help refine or improve the accuracy of toe delineation compared with Palaseanu-Lovejoy and others (2016) and provide the analyst with guidance to manually vectorize areas where cliff position estimates are erroneous.

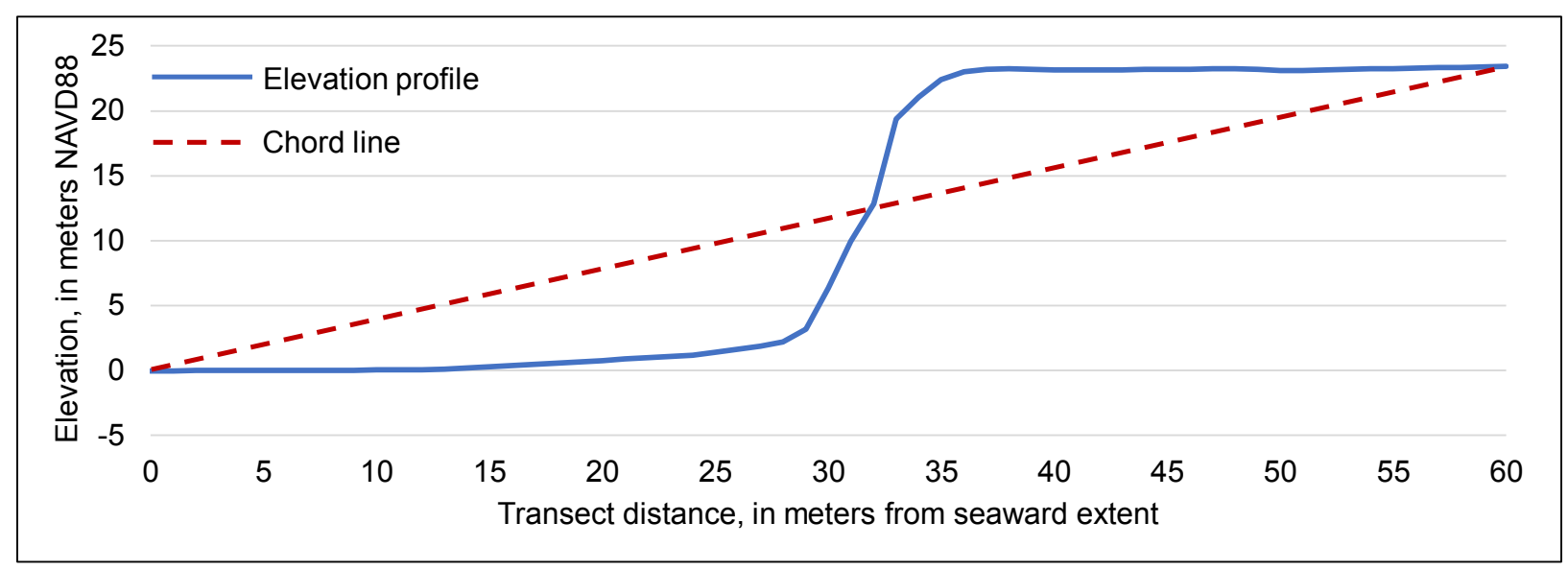

Figure 1. An elevation profile showing the upward sloping chord line projected across each transect, specifically developed by Palaseanu-Lovejoy and others (2016) for cliff feature delineation applications. NAVD 88, North American Vertical Datum of 1988. 
The CFDT uses a hybrid approach that synthesizes the Palaseanu-Lovejoy and others (2016) and Hapke and others (2018) algorithms with additional processing modifications, building on the fundamental Palaseanu-Lovejoy chord line method. Previous attempts at cliff feature delineation required a preexisting shoreline vector, or integrated DEM information only after cross-shore transects or baselines had been digitized or cast by the user, with inconsistencies in manual digitization providing opportunities for error accumulation. The CFDT analyzes the DEM during the automated generation of an offshore baseline and cross-shore transects, resulting in consistent distances between the baseline and cliff face, in addition to the establishment of landward-seaward orientation without user intervention. The tool includes 3D data review functionality, two toe-location refinement methods (one based on an edge-enhancement filter and the other on a slope-analysis kernel), a method for delineating subsidiary features on the cliff face, options for processing across a range of input DEM resolutions, and recursive error detection on the polyline outputs of cliff top and toe features. Toe refinement methods and subsidiary feature delineation can identify talus features and concavities-convexities on the cliff face, and they can provide several versions of a cliff toe for a given length of cliff. Because cliff morphology is variable and a single method may not optimally characterize all cliff types, an analyst can display multiple outputs for an overarching area and choose the one that performs best in a given region.

\subsection{Experimental Software Use Considerations}

The CFDT is intended for experimental use rather than for immediate use in aiding management decisions. The tool contains multiple methods for delineating primary and subsidiary cliff features and this guide contains no quantitative assessment of cliff feature delineation accuracy, either absolute or comparative. One method is based on the logic contained in PalaseanuLovejoy and others (2016), and the accuracy of outputs using this method is likely to be similar to the accuracy of the results reported in that study. However, the toe refinement and subsidiary feature delineation methods used by this tool diverge significantly from those of PalaseanuLovejoy and others (2016). Furthermore, a qualitative investigation of initial results suggests that the comparative performance of delineation methods is influenced by cliff morphology and foreshore slope, which are discussed further in sections 2.3 and 2.4 of this guide. CFDT users should employ quality assurance and control procedures before tool outputs are used to inform major management decisions, and they should conduct an accuracy assessment with ground-truth data when possible. Any use of trade, firm, or product names in this manual is for descriptive purposes only and does not imply endorsement by the U.S. Government.

\section{Algorithm Logic}

The delineation workflow begins after a user imports a raster DEM and an offshore baseline, with the baseline located just offshore of the cliff, generated either using the Baseline Builder Tool or through manual digitization (described in section 6). The user then populates the setting options in the tool dialog box and initiates the processing run (described in section 5). The sections below describe the logical ruleset governing cliff feature delineation in the algorithm. Explanation of the specific Python functions used to construct the algorithm logic can be found in the inline comments annotating each function in the tool's script file. 


\subsection{Generating Cross-Shore Transects}

The CFDT must first determine the correct landward or seaward orientation, relative to the offshore baseline, in order to cast cross-shore transects and extract elevation profiles of the seacliff. In this application, cross-shore transects are most effective when they are extended in the landward and seaward directions from the input baseline, with the landward component necessarily longer. Additionally, several challenges are present. First, the angle bearing of the baseline can vary widely across a given study area. Second, default topological rules that enforce the start or end of digitized lines do not account for topographic context. Lastly, single-orientation parameters input by a user are unlikely to result in accurate transect casting directions across the whole of a study area with varying shoreline orientations. The algorithm, to address these challenges, automatically determines the landward and seaward orientation on a segment by segment basis using the input DEM. Given an offshore baseline input (generated by either the user or the Baseline Builder Tool detailed in section 6), the algorithm establishes a buffer on the right and left sides of each baseline segment ${ }^{2}$ and records the mean DEM value within the buffer. For each left-right buffer pair, the buffer with the highest mean elevation indicates the landward direction (fig. 2A).

Next, CFDT uses a feature in the ArcMap library called "Linear Referencing" ("Create Route" and "Create Route Event Layer" tools, Esri ArcMap) to project a framework of points representing the start and end vertices of the seaward and landward components of transects cast perpendicular to the baseline. Points for both seaward and landward transect distances are initially projected on both sides of the baseline (fig. $2 B$ ) but are then spatially filtered by the buffer polygons (fig. 2C). This filtering step enforces the landward-seaward orientation of the points, with cross-shore transect polylines then generated from the filtered points ("Point to Line" tool, Esri ArcMap) (fig. 2D). The linear referencing and orientation workflow also allow specific locations along a given cross-shore transect to be referenced relative to the distance (in meters [m]) from the seaward start of a transect. Later steps in the algorithm, which identify cliff features, flag the feature locations using this relative distance metric. ${ }^{3}$ Transects are assigned unique identification numbers in their shapefile attribute table that represent cross-shore transect locations, in meters, along the full offshore baseline. The user specifies the spacing between transects (see section 5.2.6 for information on how to choose a transect interval).

\subsection{Extracting Surface Information Across Cross-Shore Transects}

After cross-shore transects are cast, surface information derived from the DEM is extracted along the length of each cross-shore transect and exported in tabular format. The first data element is an elevation profile interpolated (by bilinear method) directly from the DEM at an interval matching its cell resolution ("Stack Profile" tool, Esri ArcMap). A linear equation is appended to the row entries for each cross-shore transect, representing each transect's primary chord line. The chord line construction follows the same logic as Palaseanu-Lovejoy and others (2016); the polynomial is formed by connecting the most seaward $x y$ coordinate on the elevation profile with the most landward $x y$ coordinate (fig. 1). At this stage, any transect that lacks

\footnotetext{
${ }^{2}$ These segments are defined as continuous, unbroken polylines.

${ }^{3}$ For more information on linear referencing see https://desktop.arcgis.com/en/arcmap/10.6/ manage-data/linear-referencing/what-is-linear-referencing.htm
} 


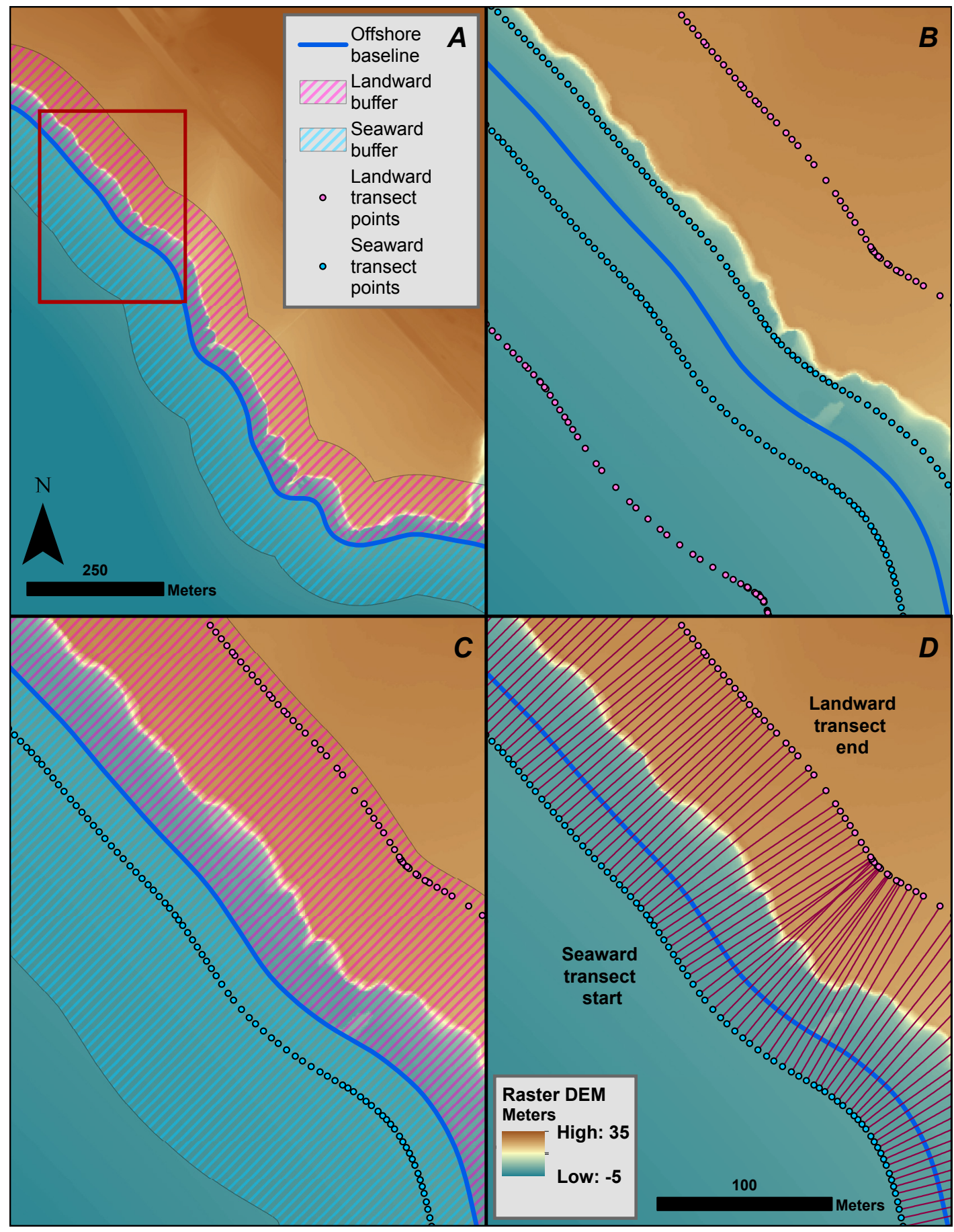

Figure 2. Maps showing the step-by-step process used by the algorithm to generate crossshore transects. Images showing $(A)$ elevation scanning buffers projected from offshore baseline segments. These buffers identify the landward (pink) and seaward (blue) orientation adjacent to each segment. Only one baseline segment is shown in this example, but a typical processing run would include multiple segments. Red box identifies location of images in $B, C$ and $D$. (B) Points representing the start and end vertices of landward and seaward transect components before orientation filtering. $(C)$ Seaward and landward points remaining after orientation filtering. (D) Cross-shore transects formed from filtered seaward and landward points. 
elevation data at its start or endpoint is removed from processing, as this indicates that the DEM does not encompass the full transect extent.

The second element of extracted data is a tabular high-pass profile used to refine cliff toe delineation (fig. 3A). A surface-slope raster is first generated from the DEM ("Slope" tool, Esri ArcMap) using a $3 \times 3$ cell kernel that calculates the rate of elevation change to derive these data. ${ }^{4}$ Next, this slope raster is processed by a high-pass filter ("Filter" tool, "High" setting, Esri ArcMap), which applies a modified 3x3-cell Laplace kernel. ${ }^{5}$ High-pass filters are image-analysis operators used to accentuate the edges between value gradients in rasters, with filter outputs that are signed, unitless values. In this context, the high-pass filter produces a new raster with extreme positive and negative values around the intersection of two planar surfaces (fig. 3B). Extreme positive values are on the upper side of the edge, and extreme negative values are on the lower side of the edge. Values on the planes separate from the planar intersections remain close to zero. The negative values on the high-pass filter raster are tabulated and appended to the elevation profile (fig. $3 A$ ) ("Stack Profile" tool, Esri ArcMap), with positive values excluded, as they are not used later. While this tool uses a bilinear interpolation when it extracts data from rasters and changes the high-pass values, the spatial location of local high-pass maxima does not change.

\subsection{Locating Cliff Tops, Midpoints, and Basic Toes}

Cliff top and basic toe delineation occur using logic similar to that established in Palaseanu-Lovejoy and others (2016). For these analyses, the vertical distance between the chord line and the surface profile is calculated for every extracted cell (row) along the cross-shore transect. The cell entry with the greatest positive difference from the chord line is identified as the cliff top, and the entry with the greatest negative vertical distance from the chord line is identified as the basic cliff toe (fig. 4). After the cliff top and basic toe are determined, the cliff midpoint is flagged by identifying the location on the cross-shore transect between the cliff top and basic toe where the surface elevation is closest to half of the cliff height, or:

$$
\left(\frac{\text { Cliff Top Elevation }}{2}\right)+\text { Cliff Toe Elevation }
$$

The cliff midpoint is useful for distinguishing between physical processes affecting the upper and lower halves of cliffs, and it is also used later during cliff toe refinement and subsidiary feature delineation.

\subsection{Refining the Cliff Toe Location}

The basic chord line distance method described above yields strong position estimates for the cliff top and toe when cliffs in a study area are relatively high relief, and cross-shore transect lengths can be relatively short. These factors allow for a steeper primary chord line that is more likely to have a slope exceeding that of the beach fronting the cliff. However, when cliff faces are more gradually sloped or transect distances must be made longer, the basic toe delineation may become less accurate. This problem occurs when the slope of the foreshore approaches or

\footnotetext{
${ }^{4}$ For more information on the slope algorithm used in the Esri slope tool, see https://desktop. arcgis.com/en/arcmap/10.6/tools/spatial-analyst-toolbox/how-slope-works.htm

${ }^{5}$ For more information on the high pass filter algorithm used in the Esri ArcGIS "Filter" tool, see https://desktop.arcgis.com/en/arcmap/10.6/tools/spatial-analyst-toolbox/how-filter-works.htm
} 


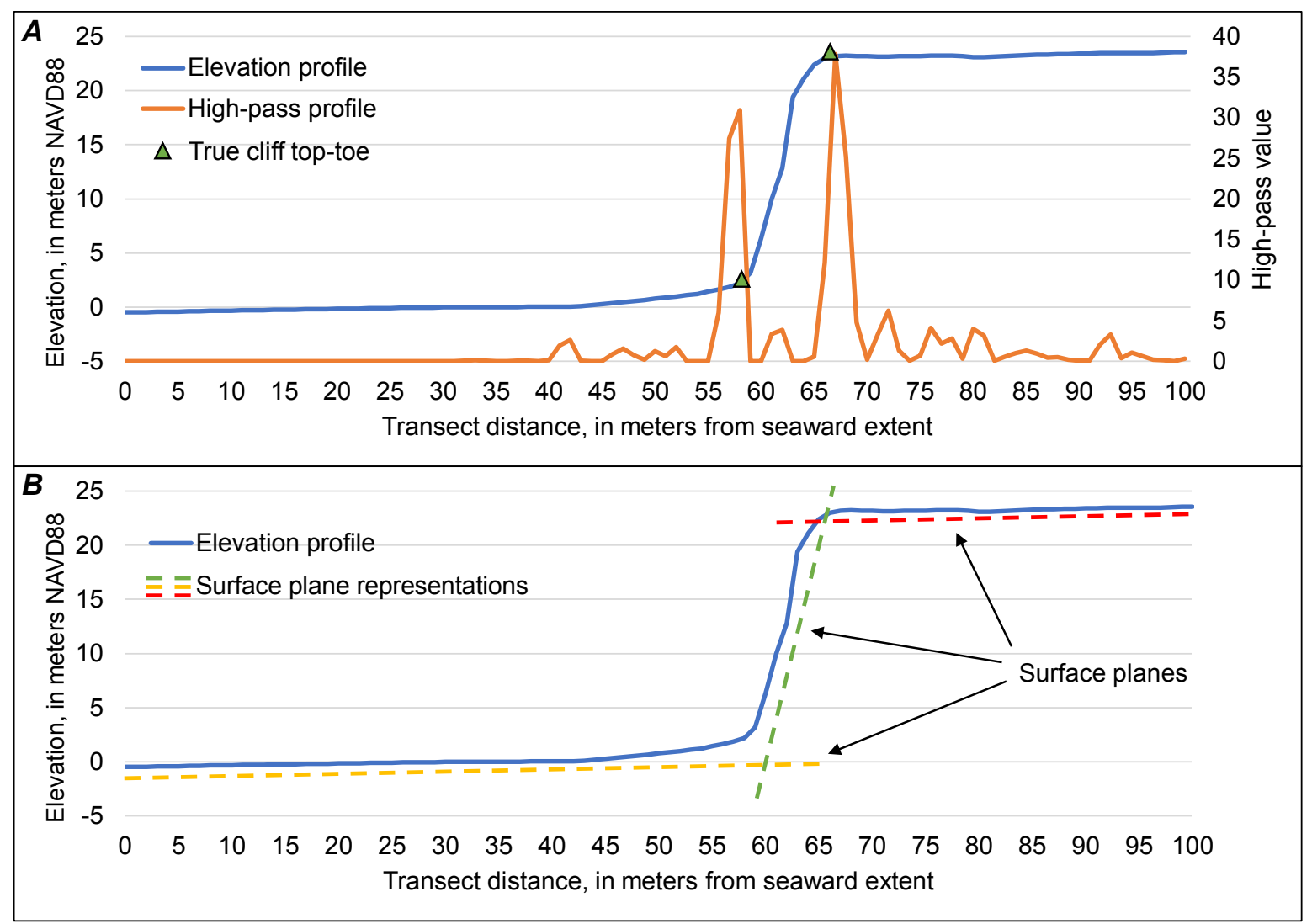

Figure 3. Cliff elevation profiles showing $(A)$ a corresponding high-pass profile along a cross-shore transect and $(B)$ variously colored dashed-line representations of rough surface planes occurring along the cross-shore transect. High-pass value extrema correspond to surface-plane intersections and are more extreme when slope differences between two planes are large and occur over a short horizontal distance. NAVD 88, North American Vertical Datum of 1988.

exceeds the slope of the chord line so that the true cliff toe location has a smaller vertical difference from the chord line than areas of the foreshore (fig. 4). For this reason, when basic toe misclassifications occur, they usually generate false positives on the foreshore and occasionally on the landward extent of the talus. Because cliff morphology is likely to be variable across a study area and it is impractical for a human analyst to optimize cross-shore transect lengths for every transect interval, two cliff toe refinement methods that can improve the cliff toe delineation automatically, without modifying cross-shore transect length, are included.

First, the "High Pass" method scans the high-pass profile for values that exceed a userspecified threshold (fig. $3 A$ ), restricting the processing extent to the cross-shore transect area between the basic toe and midpoint. This threshold is a unitless, negative high-pass value ranging from -6 to -50 and defaulting to -25 in the absence of user input. Starting at the basic toe and moving toward the midpoint, the first cell along the transect with a value that exceeds (has a more extreme negative value than) the high-pass threshold is flagged as the refined cliff toe. If the user sets the high-pass threshold closer to zero, the refined toe is classified on surface-plane intersections that are less different in slope and usually farther seaward. If the algorithm scans the region between the basic toe and midpoint without triggering the threshold, the threshold 


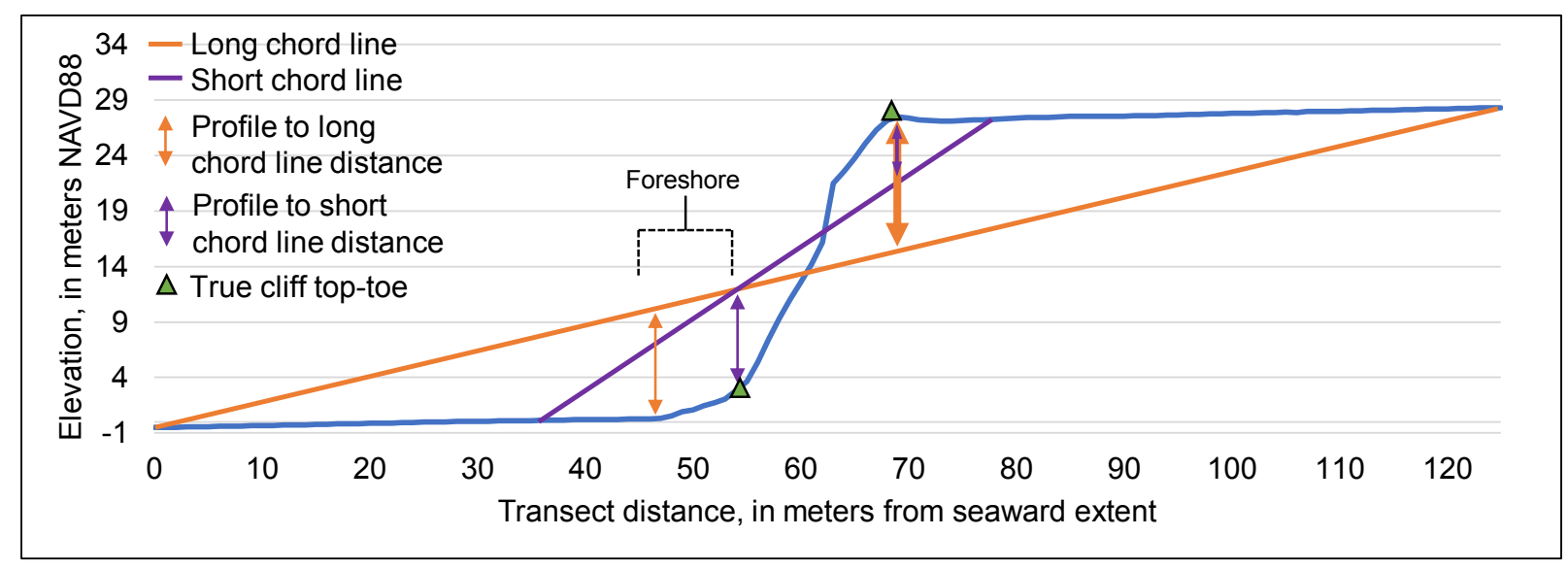

Figure 4. A surface profile showing chord line length as a potential source of cliff feature misclassification. The differences between the chord line and cliff top-toe predictions (profile to chord line distance maxima) are shown for short and long transects. The foreshore slope exceeds the chord line slope for the long transect, and as a result, the toe is identified seaward of its true location. In this example, the cliff top position is tolerant of variability in the slope of the chord line. NAVD 88, North American Vertical Datum of 1988.

is revised five units ${ }^{6}$ closer to zero and the region is rescanned starting from the basic toe. This process iterates until the threshold is exceeded or the revised threshold reaches -5 , as values less extreme than -5 represent profile features too small to be cliff toes. In the latter case, the basic toe is accepted as the refined toe. A user-modifiable, downward-revising high-pass threshold helps compensate for circumstances where increases in the slope across the cliff toe region are subtle. In these situations, the high-pass profile values around the toe may be too low to exceed the default threshold (fig. $5 A$ ) or may be less extreme than planar intersections higher on the cliff face between the basic toe and midpoint (fig. 5B).

Second, the "First Concavity" method identifies the cliff toe by casting a secondary chord line formed by a linear function connecting the basic toe and the cliff midpoint and then running an analysis kernel across the elevation profile (fig. 6). The analysis kernel is one-dimensional (a single row of cells), and the user can modify its size (length in cells). The kernel computes averages of the local surface slope, flagging points where the slope of the surface profile dips below and rises above the slope of the secondary chord line within the kernel's extent, representing a concavity on the elevation profile (fig.6). The most seaward flagged cell between basic toe and cliff midpoint becomes the refined toe position. If no convexity is found on the lower half of the cliff, the refined toe location reverts to the basic toe location.

Both toe refinement methods are restricted to regions of the cliff between one cell seaward of the cliff toe and the cliff midpoint in order to avoid the generation of false positives on other shoreline or bathymetric features. When basic toe misclassification occurs, toes are most frequently identified seaward of the true toe, and refinement methods perform best in these situations; an accurate basic toe may limit refinement analysis to areas on the cliff face above the cliff toe. Thus, when cross-shore transects are short (such as on simple, high-relief cliffs), refined toes may appear on the cliff face, and the basic toe delineation may outperform the refined delineation.

${ }^{6}$ The five-unit interval was selected as a compromise between smaller intervals-smaller intervals require more iterations to classify a cliff feature, which consumes more processing time. 


\subsection{Subsidiary Feature Delineation}

The cliff top, toe, and midline are not the only features on a cliff that have implications for how and why a coastal cliff erodes. Convexities and concavities on a cliff face could indicate a break in a slope resulting from differential erosion of the cliff, the talus debris fan, or the intersection of the talus debris fan with the cliff face. To delineate these features, secondary chord lines are cast on both the upper and lower halves of the cliff. A linear chord connects the basic toe and midpoint, and a separate linear chord connects the midpoint and cliff top (fig. 6). The same slope-measurement kernel used for "First Concavity" toe refinement then scans the upper and lower portions of the cliff separately. Points are flagged any time the analysis kernel detects slopes, across its extent, that dip below and then rise above the given chord line (fig. 6), representing a surface concavity (potentially the intersection of a talus debris fan with the cliff face). Points are also flagged when the slope rises above and then falls below the given chord line, representing a surface convexity (potentially a differential erosion feature on the cliff face or a seaward portion of the talus debris fan). Unlike toe refinement, the kernel scans the full extent of the upper and lower halves of the cliff, flagging all surface convexities and concavities detected.

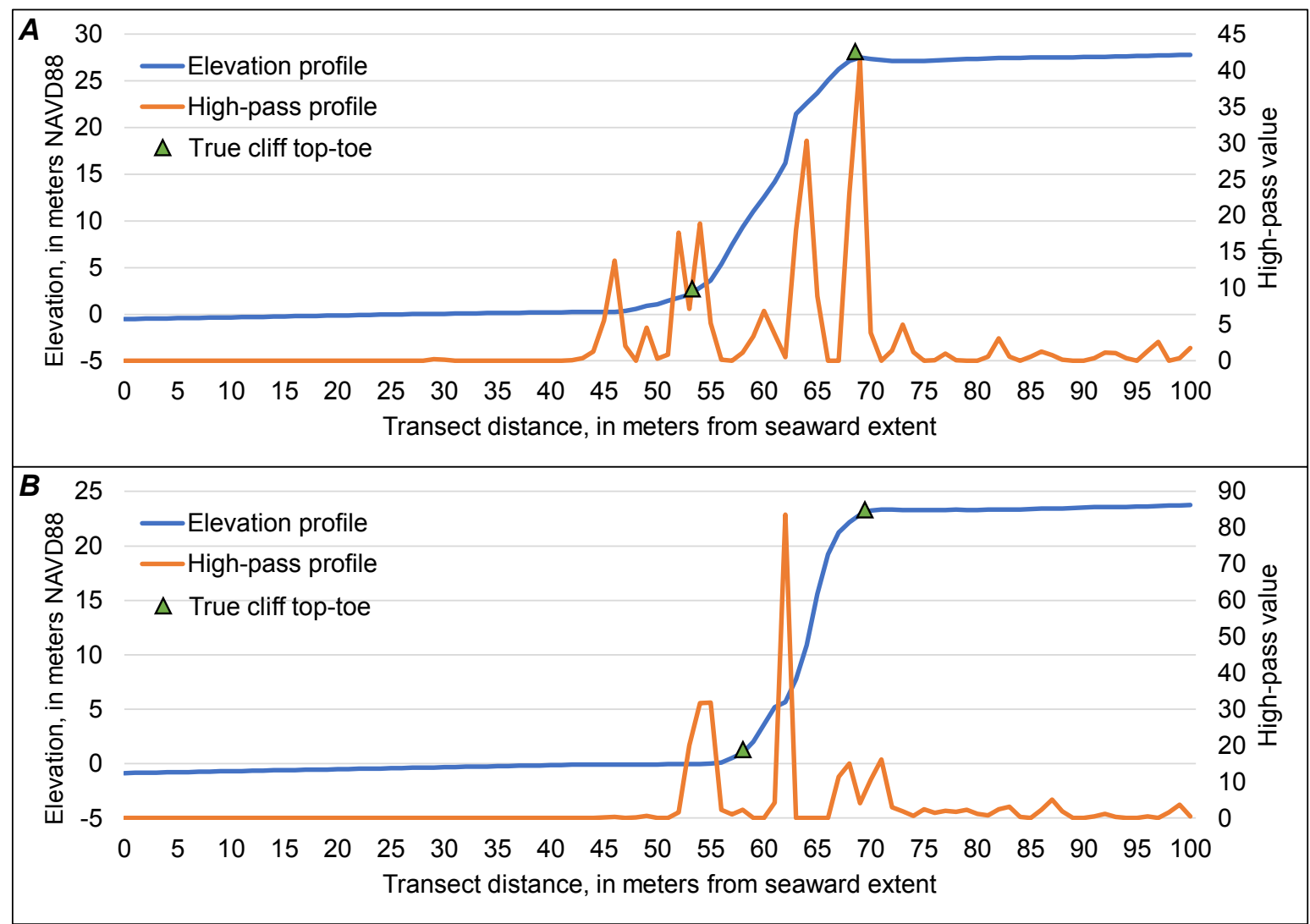

Figure 5. Surface profiles showing examples of potential errors during high pass method toe refinement. Graphs showing $(A)$ elevation and high-pass profiles in circumstances where a default -25 high-pass threshold will fail to classify a cliff toe, and $(B)$ another feature on the lower half of the cliff face having a more extreme high-pass value than the cliff toe. The high-pass value does not have measured units. NAVD 88, North American Vertical Datum of 1988. 


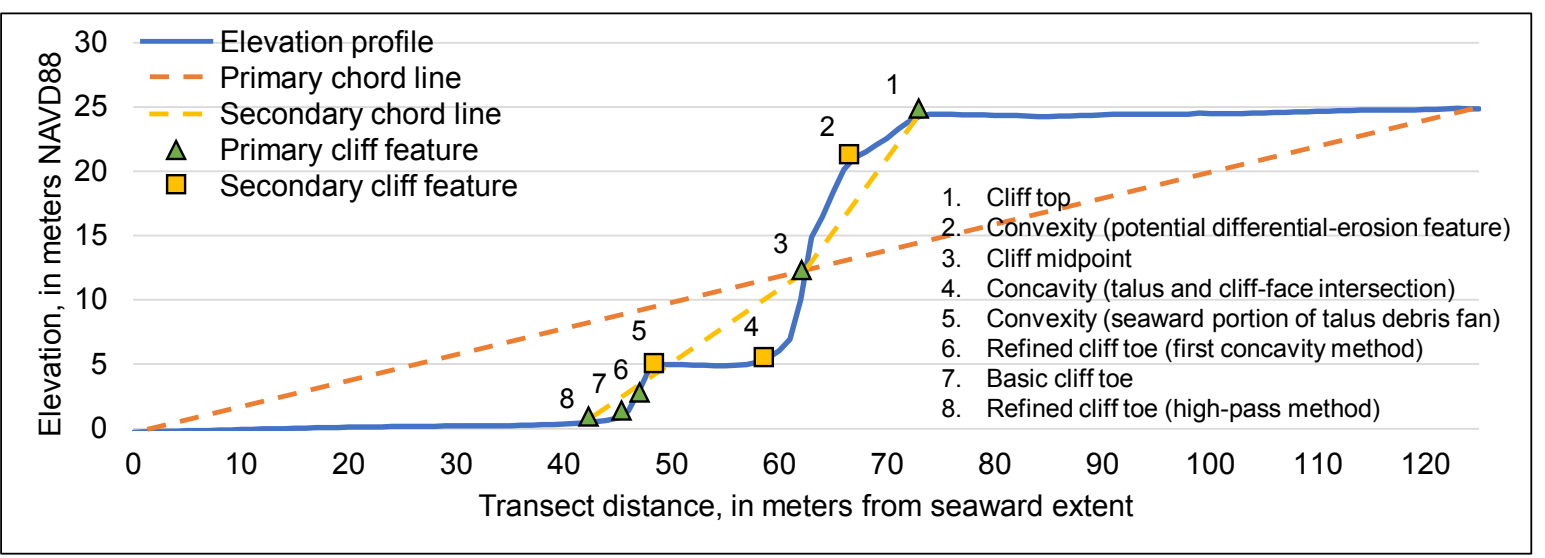

Figure 6. A surface profile showing the possible outputs using the Cliff Feature Delineation Tool. Elevation profile with secondary chord lines and subsidiary features shown with the primary chord line and primary cliff features, including a basic toe and refined toes. Subsidiary features represent convexities and concavities on the cliff face relative to the subsidiary chord lines and could indicate geologic controls on cliff morphology. NAVD 88, North American Vertical Datum of 1988.

The flagged convexities and concavities are then filtered separately by the vertical distance from their respective chord lines; the convexity with the greatest positive vertical distance from the secondary chord line and the concavity with the greatest negative vertical distance are selected as subsidiary features. This process occurs for both the upper and lower halves of the cliff, resulting in a maximum of four subsidiary features possible on a given transect. For Basic Delineation mode, an additional subsidiary feature can be identified on the lower half of the cliff to allow for the toe of the talus (or the true cliff toe) to be identified in circumstances where the coarse toe is misclassified seaward and fails to capture its true location.

For both "First Concavity" toe refinement and subsidiary feature delineation, the user can modify the size of the slope-analysis kernel. The optimal kernel size depends on cliff morphology and the input DEM resolution. Modifying the kernel size can make delineation less likely to generate false positives on small slope features that are not explanatory for the scale of study or can parameterize a finer-scale kernel for use on high-resolution DEMs. For instance, if an input DEM with a $0.1-\mathrm{m}$ cell resolution is used as an input, a 3-cell kernel only evaluates $0.3-\mathrm{m}$ portions of a cliff at a given time - this cross-shore length is unlikely to encompass the extent of a significant cliff feature on any but the smallest cliffs and instead represents numerous surface deviations that do not represent overarching cliff morphology. However, a 30-cell kernel encompassing a 3-m cross-shore length aggregates slope measurements over a larger area, diminishing the influence of slope deviations across small linear distances and correlating measurements with larger, more relevant cliff features.

\subsection{Vectorizing Cliff Features}

In the two previous sections, cliff features were identified in a tabular format. However, every cell-row extracted from a cross-shore transect and identified as a cliff feature remains referenced to its one-dimensional location on a given cross-shore transect. Thus, all delineated features can be reprojected as point-feature shapefiles. A linear referencing operation ("Create Route" and "Make Route Event Layer" tools, Esri ArcMap) is used to project point shapefiles, 


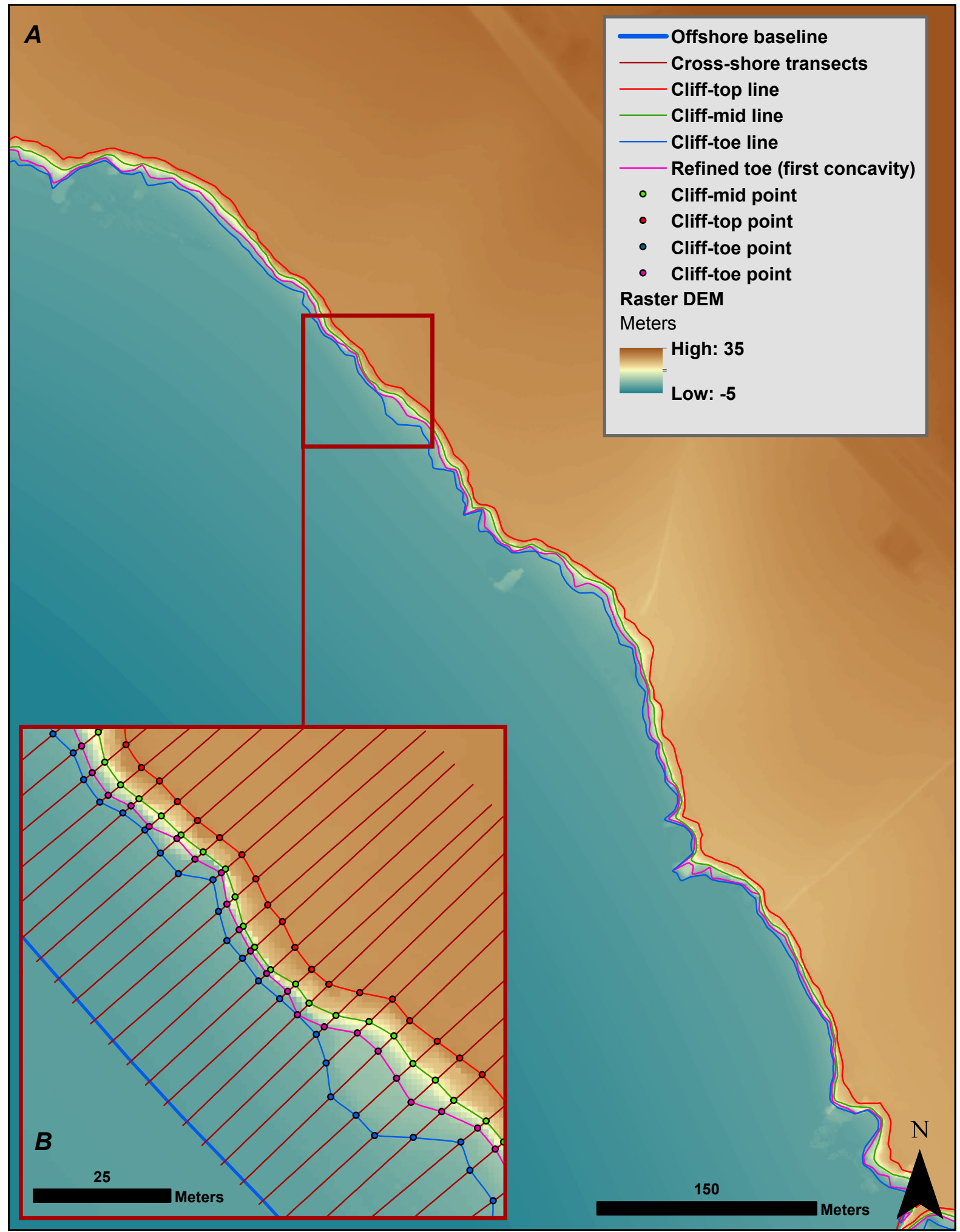

Figure 7. Images showing $(A)$ linear cliff features constructed alongshore, including a cliff top, cliff midline, cliff toe, and refined cliff toe generated using the "First Concavity" method, and $(B)$ linear cliff feature lines converted from point features referenced to a one-dimensional position along a given cross-shore transect. DEM, digital elevation model. 
which are then converted to lines ("Point to Line" tool, Esri ArcMap) (fig. 7). A separate polyline shapefile is output for the cliff top, midpoint, and toe (or multiple toes if Multi Delineation mode is selected). Primary cliff features are additionally generated as point shapefiles, along with subsidiary features. Cliff feature lines are broken wherever a user leaves gaps after digitizing the offshore baseline, and gaps are also left in areas where the feature delineation failed to generate valid outputs. For instance, if an error or exception occurs during delineation of the coarse toe, midpoint, or top, the algorithm cancels delineation and skips to the next transect because these initial delineations are prerequisites for any location refinement or subsidiary feature delineation.

If offshore baselines were not digitized in accordance with proper protocols (outlined in section 6), cliff feature lines might cut back on themselves alongshore. The CFDT checks for remaining cutbacks by intersecting cross-shore transects with cliff feature lines and flags transects that cross over a given feature line more than once. Depending on the user-defined settings, transects are buffered and either erase underlying transects or simply flag transects where cutbacks occur. It is common to encounter cutback transects around baselines that surround narrow headlands or cliffs on shorelines with short alongshore crenulations.

Once all features are converted to line shapefiles and erroneous sections are removed, all cliff feature lines, subsidiary feature points, and cross-shore transects are converted to 3D vectors for viewing in 3D visualization software like ArcScene. The input DEM is treated as an elevation benchmark over which feature lines and points are interpolated ("Interpolate Shape" tool, "Bilinear" method, Esri ArcMap), resulting in feature lines that conform closely to the DEM surface. Importantly, only the $z$ values of the input lines are interpolated - no modification occurs to the $x$ or $y$ coordinates of the polyline. For this reason, the cliff midline, which likely falls on the face of a high-relief surface, may appear to have a high variance between cross-shore transects as it conforms to the face of the cliff between midpoint positions on transects (fig. 8). This is entirely normal and is a product of the alongshore resolution of outputs (the Transect Interval chosen by the user).

\subsection{Cleaning the Scratch Workspace}

At several points in the script, the algorithm scans the scratch workspace to delete intermediate files generated during processing. These files can number in the thousands and are associated with route-merging processes when tabular features are converted to shapefiles or with erase processes that clean up erroneous sections of cliff feature lines. The algorithm also deletes these files if an exception is encountered that prevents the tool from completing its processing run. This step is critical; if these files persist after a given tool run, the intermediate outputs left in the scratch workspace are integrated into the next tool run, which causes erroneous projected feature lines and unexpected gaps in datasets where valid data was erased. For this reason, if the computer restarts unexpectedly, or if ArcMap closes unexpectedly before a processing run can complete, the user should delete all files remaining in the "Scratch" folder to ensure they are not inadvertently integrated into the next batch of outputs. 


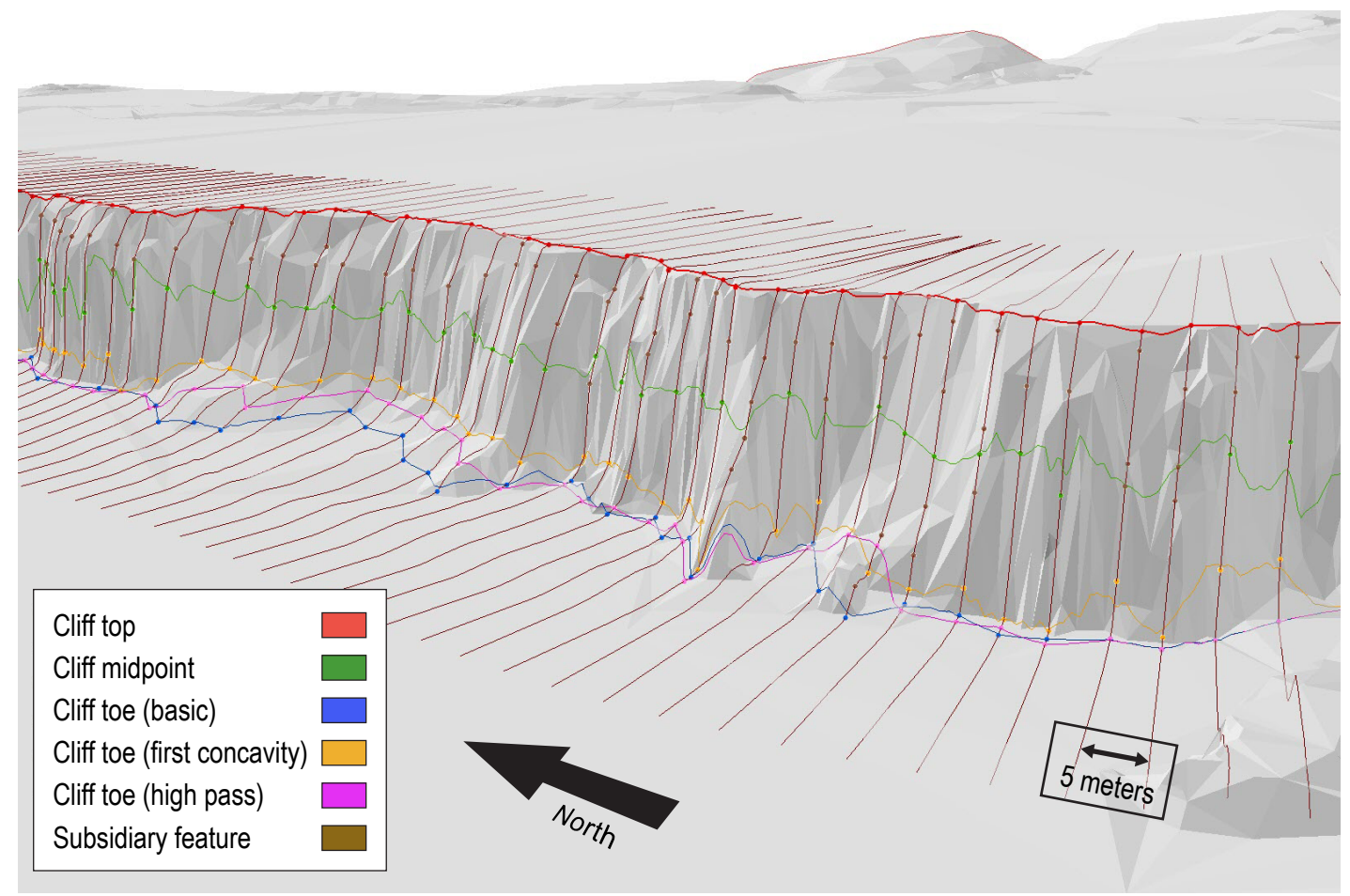

Figure 8. An image showing all possible outputs of the Cliff Feature Delineation Tool shown for a short stretch of coast in the ArcScene 3D format, including the cliff top (red), midline (green), toe (blue), refined toe from the high-pass filter method (pink), and refined toe from the first concavity method (orange). Cliff point features are shown in colors corresponding to these lines. Subsidiary point features are shown as brown points, and cross-shore transects are displayed in dark red. A triangular irregular network (TIN) derived from the input raster digital elevation model (DEM) is displayed as the backdrop. 3D, three-dimensional.

\section{Installation}

\subsection{System Requirements}

CFDT v1.0 requires the following to run:

1. Microsoft Windows 7 or Windows 10 operating system.

2. ArcGIS Desktop Standard license or higher. This tool was developed for use with ArcGIS Desktop version 10.6 and 10.7.
A. ArcGIS Desktop Spatial Analyst and 3D Analyst extensions,
B. ArcGIS Desktop .NET support feature (installed by default), and
C. Microsoft .NET framework of 4.2.2 or later (installed by default).

\subsection{Unpacking the Tool}

The Cliff Feature Delineation Tool and companion Baseline Builder Tool exist as Python scripts within an ArcGIS toolbox, along with a preestablished set of folders in a working directory. Follow the steps in figure 9 to set up the tools for use. 


\begin{tabular}{|c|c|}
\hline Software setup steps & Visual guidance \\
\hline 1. Download the CFDT .zip folder. & Download link: https://doi.org/10.5066/P9UKW7PO \\
\hline $\begin{array}{l}\text { 2. Extract the .zip folder to the chosen di- } \\
\text { rectory. This directory is where final and } \\
\text { intermediate outputs are stored and should } \\
\text { have several gigabytes of free storage space } \\
\text { available. }\end{array}$ & 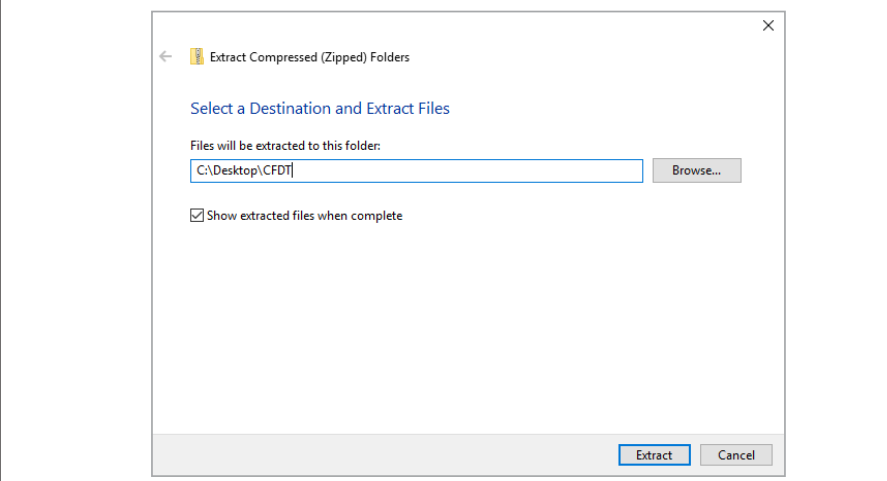 \\
\hline 3. Open ArcMap. & \\
\hline $\begin{array}{l}\text { 4. From the main menu, open the "Catalog" } \\
\text { pane. }\end{array}$ & 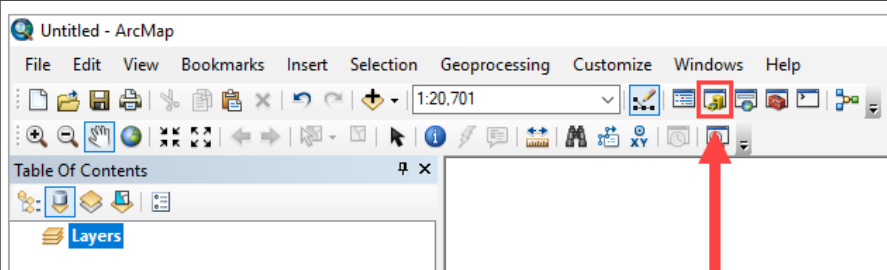 \\
\hline $\begin{array}{l}\text { 5. Click the "Connect to Folder" button in the } \\
\text { "Catalog" pane, then navigate to the CFDT } \\
\text { directory extracted in step } 2 \text {. Select it and } \\
\text { click "OK." }\end{array}$ & 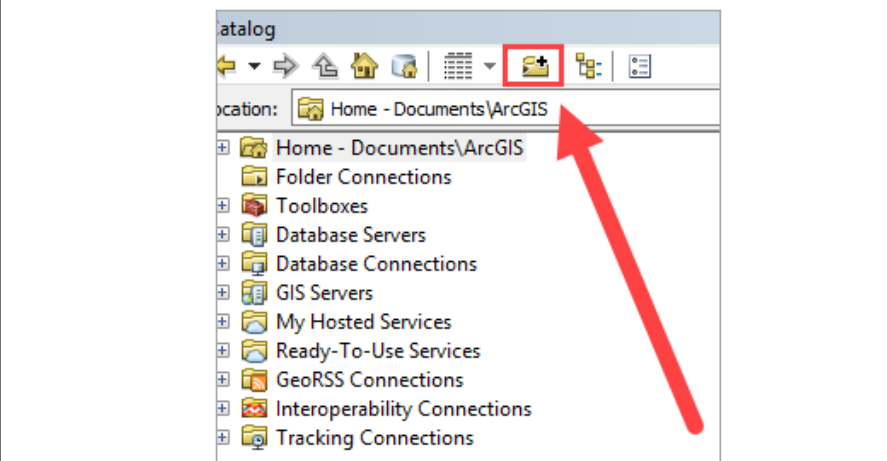 \\
\hline $\begin{array}{l}\text { 6. The CFDT directory should now appear un- } \\
\text { der the "Folder Connections" section of the } \\
\text { "Catalog" and can be accessed by ArcMap. }\end{array}$ & 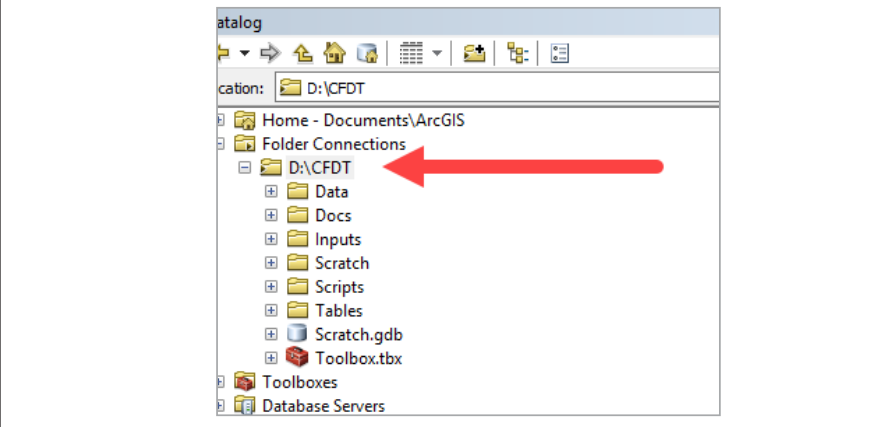 \\
\hline
\end{tabular}

Figure 9. Instructions for setting up the Cliff Feature Delineation Tool (CFDT) and companion Baseline Builder Tool within the ArcGIS toolbox. .zip, lossless data compression archive file. 


\subsection{Working Directory Structure}

The directory extracted during section 3.2 contains the following files and folders required for the tool to operate:

- Data: Final feature delineation outputs are saved to this location. The user can delete files contained in this directory. Note that storing other files in this directory does not cause errors.

- Scratch: Intermediate rasters and shapefiles generated during processing are saved to this location. Most of these files are deleted at the end of a processing run, but some are left to aid the user in troubleshooting unexpected problems detected in the results. Intermediate results are overwritten during each processing run. The user may delete files contained in this directory. However, storing any additional files to this location may cause errors to develop in tool outputs.

- Scratch.gdb: Intermediate geodatabase files are saved to this location. The user may delete files contained in this geodatabase. However, this should be done through the ArcGIS Catalog, not through Windows File Explorer. The user should not store any additional files to this location.

- Tables: All intermediate tabular files are saved to this location. These files remain after processing to aid the user in troubleshooting unexpected problems detected in the results. These tables are overwritten during each processing run. The user may delete files contained in this directory. The user should not store additional tables in this location.

- Scripts: The Python scripts containing the operating code for the CFDT and Baseline Builder Tool are stored in this location. These files should not be moved or modified in any way unless the user is attempting to change the tools' functionality.

- Toolbox.tbx: This ArcGIS toolbox is the intermediary between the Python script in the Scripts folder and the ArcMap interface that allows the user to enter tool run parameters and execute processing runs. The toolbox should not be moved or modified.

- Docs: Tool "readme" documents are stored in this location, including this user manual and example argument text (.txt) files for executing the CFDT and Baseline Builder Tool outside of the ArcMap environment. Original files that are unpacked with the tool in this directory should not be deleted as the user may require them for reference. Other files may be stored in this directory.

- Inputs: This directory is provided as a location to store DEM and offshore baseline datasets used as initial tool inputs. Use of this folder is optional and users may add, remove, or modify files in this folder as desired.

Except for the "Inputs" folder, the CFDT and Baseline Builder Tools reference the above folders by pathname. Modifying the names of any folders causes the tool to fail. Adding other folders to the directory is acceptable. 


\section{Input Data Requirements}

Two datasets are required for the CFDT to run: a raster DEM and an offshore baseline. Requirements for the datasets appear in this section.

\subsection{The Raster Digital Elevation Model}

- The "Raster DEM" is a raster digital elevation model (DEM) in Georeferenced Tagged Image File Format (.geotiff) that characterizes the topography on and around the seacliffs (fig. 10).

- Topobathymetric or topographic DEMs may be used. In either case, data values must exist at the seaward and landward extent of cross-shore transects. If there is insufficient DEM coverage on transect extents, the user can use ArcMap's "Euclidean Allocation" and "Mosaic to New Raster" tools to extend the DEM with proxy values drawn from the DEM's edge. ${ }^{7}$

- Any patches of "Null" or "NoData" values within the data extent of the raster DEM should be filled; otherwise, cliff features are delineated erroneously. The user can apply raster extension techniques, such as ArcMap's "Euclidean Allocation" and "Mosaic to New Raster" tools, to fill these areas. Additionally, if a hydro-flattened DEM is available for the study area, the voids are already filled.

- The CFDT accepts DEMs in any spatial resolution, including those derived from traditional aerial lidar and small unmanned aerial system (sUAS) missions. During CFDT testing, 1.0- and 0.5-m resolution DEMs were appropriate to delineate the talus and subsidiary cliff features on seacliffs $20-50 \mathrm{~m}$ tall-coarser resolutions may be acceptable for larger cliffs, and finer resolution may be beneficial for smaller cliffs. Generally, if a DEM's resolution is too coarse for the user to recognize an existing talus or convexities-concavities on an elevation profile of a cliff face, finer resolutions (if available) can improve delineation.

- It is not advisable to substitute a digital surface model (DSM) for the DEM. A DSM includes elevation values representing foliage and built structures. These elements may cause cliff feature misclassifications, as the tool cannot distinguish between bare-earth elevations and built structures or trees.

- The DEM must be projected in a Universal Transverse Mercator (UTM) coordinate system, with the vertical and horizontal units in meters. Additionally, the horizontal and vertical coordinate system used must match that of the offshore baseline. Use the "ArcMap Project" tool to change the spatial reference of a DEM or baseline shapefile. ${ }^{8}$

\footnotetext{
${ }^{7}$ For more information on the "Euclidean Allocation" tool, please visit https://desktop.arcgis. com/en/arcmap/10.6/tools/spatial-analyst-toolbox/euclidean-allocation.htm. For more information on the "Mosaic to New Raster" tool, please visit https://desktop.arcgis.com/en/arcmap/10.6/ tools/data-management-toolbox/mosaic-to-new-raster.htm.

${ }^{8}$ For more information on the Esri ArcGIS “ArcMap Project” tool, see https://desktop.arcgis. com/en/arcmap/10.6/tools/data-management-toolbox/project.htm.
} 


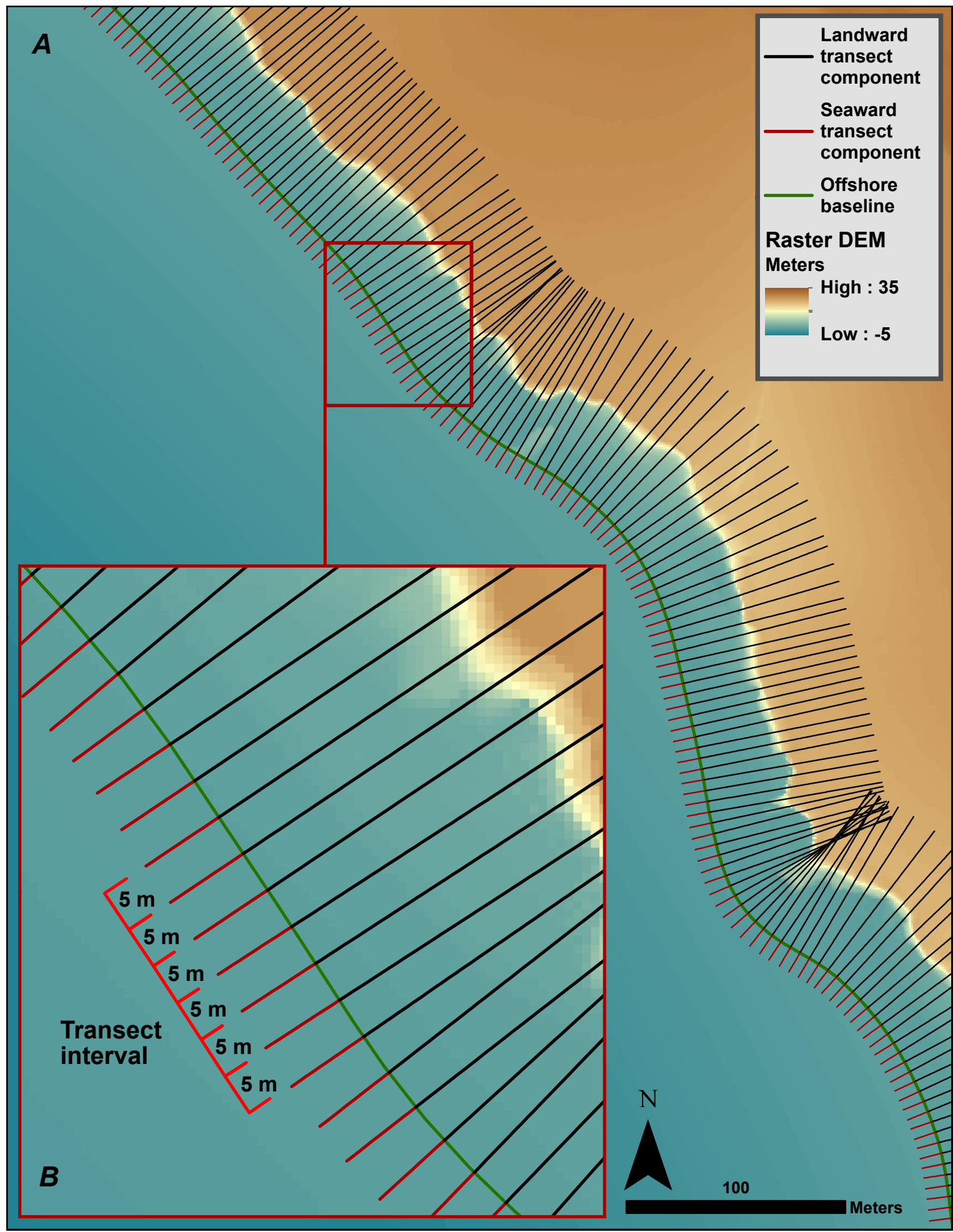

Figure 10. Images showing $(A)$ the raster digital elevation model (DEM) with an overlying offshore baseline generated with the Baseline Builder Tool, along with cross-shore transects split into landward and seaward components, as specified by the distance parameters selected by the user. $(B)$ The transect interval (a 5-meter [m] interval in this example) determines the alongshore resolution of the Cliff Feature Delineation Tool (CFDT) outputs. 


\subsection{The Offshore Baseline}

- The Offshore Baseline input is a polyline running alongshore and seaward of the seacliff features to be delineated (fig. 10). Cross-shore transects are generated perpendicular to this baseline, with the longest component cast in the landward direction.

- For obtaining the best results, this baseline should be approximately 5-30 pixels from the seaward extent of the cliff toe or talus debris fan (if one exists). The baseline setback should be considered at the same time as the settings for the Landward Distance and Seaward Distance parameters (section 5.2.4). There is no maximum length limitation on the baseline.

- Baseline segments may assume any orientation and direction. However, large changes in baseline segments' angle bearing between adjacent cross-shore transects should be minimized. Gaps should be left in the baseline where no cliff features exist, around narrow jutting headlands, and in areas where the analyst does not want tool outputs.

- The input baseline shapefile may be single or multipart, providing all entries in the attribute table have an "ID" field with the same value.

- The offshore baseline shapefile must be defined in a projected UTM coordinate system, and the spatial reference must match that of the input raster DEM. ${ }^{9}$ To change the spatial reference of a DEM or baseline shapefile, use the ArcMap "Project" tool. ${ }^{10}$

TIP: The included Baseline Builder Tool can automatically generate an offshore baseline, use a baseline from a previous dataset, or allow a new baseline to be digitized manually. Baselines generated with the Baseline Builder Tool automatically conform to projection and attribution requirements. For more information on automatically generating a baseline, see section 6 .

\footnotetext{
${ }^{9}$ To check the spatial reference of the input DEM or baseline, right-click on the raster or shapefile in the ArcMap Catalog, click "Properties," select the "General" tab, and scroll down to the "Spatial Reference $>>x y$ ” coordinate system entry.

${ }^{10}$ For more information on the ArcMap "Project" tool, see https://desktop.arcgis.com/en/ arcmap/10.6/tools/data-management-toolbox/project.htm.
} 


\section{Running the Tool}

\subsection{Initiating a Cliff Feature Delineation Tool Run}

Follow the steps in figure 11 to run the Cliff Feature Delineation Tool.

\begin{tabular}{|c|c|c|c|}
\hline Software steps & \multicolumn{2}{|c|}{ Visual guidance } & \\
\hline $\begin{array}{l}\text { 1. Open ArcMap, open the Catalog } \\
\text { pane, and expand the "Folder } \\
\text { Connections" folder. Expand the } \\
\text { CFDT directory, }{ }^{11} \text { which may } \\
\text { take a moment to process. }\end{array}$ & \multicolumn{2}{|c|}{ 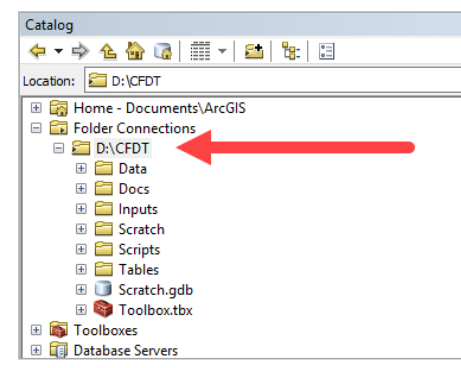 } & \\
\hline $\begin{array}{l}\text { 2. Expand the "Toolbox.tbx" file, } \\
\text { right-click on the "Cliff Feature } \\
\text { Delineation Tool," and select } \\
\text { "Open" from the dropdown list. }\end{array}$ & \multicolumn{2}{|c|}{ 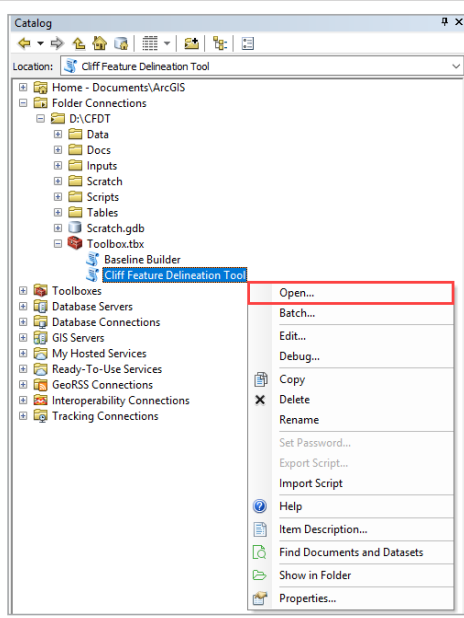 } & \\
\hline $\begin{array}{l}\text { 3. Fill out the CFTD dialog box } \\
\text { with the desired input datasets } \\
\text { and processing settings. Click the } \\
\text { "Show Help" button at any time } \\
\text { for an explanation of settings. } \\
\text { When all settings are chosen, } \\
\text { click "OK" to initiate the pro- } \\
\text { cessing run. }\end{array}$ & 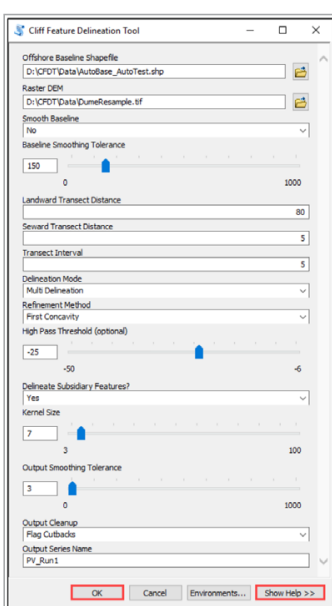 & 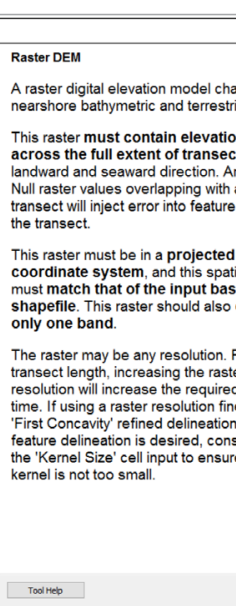 & 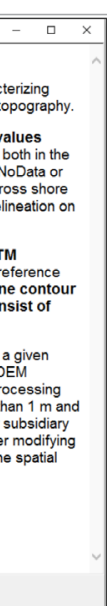 \\
\hline
\end{tabular}

Figure 11. Instructions for initiating a Cliff Feature Delineation Tool (CFDT) run.

${ }^{11}$ If the "CFTDv1" directory does not appear in the "Folder Connections" folder, follow step 3 of section 3.2 in this guide to reestablish the directory in the connected-folders list. 


\subsection{Explanation of Tool Inputs and Starting Parameters}

An explanation of tool parameters and tips on how to select input values is below. For a more detailed conceptual description on the function of parameters and associated processes, please refer to section 2 .

\subsubsection{Offshore Baseline Parameter}

The "Offshore Baseline" input is a reference polyline shapefile from which cross-shore transects are cast onto seacliffs. The user can use the browse button to navigate to, and select, a baseline shapefile. The dataset must meet the requirements detailed in section 4.2.

\subsubsection{Smooth Baseline Parameter}

The Smooth Baseline input determines whether or not the algorithm smooths the input baseline before processing. Some input baselines, such as those generated by the Baseline Builder Tool, are already smoothed and additional smoothing may not be appropriate.

- No: Baseline smoothing is not performed. This is the default option.

- Yes: Baseline smoothing is performed. The level of smoothing is determined by the Baseline Smoothing Tolerance parameter described below.

\subsubsection{Baseline Smoothing Tolerance}

The "Baseline Smoothing Tolerance" parameter is optional and specifies the extent of smoothing performed on a baseline before processing, assuming the user selected "Yes" for the "Smooth Baseline" option described above. The input, which represents meters, can be any integer between 0 and 1,000. The relative influence of high (larger input) and low (smaller input) smoothing tolerances is displayed in section 6.2.

\subsubsection{Raster DEM Parameter}

The "Raster DEM" parameter specifies the DEM characterizing the seacliffs to be delineated. The user can use the browse button to navigate to, and select, a DEM .geotiff. The dataset must meet the requirements detailed in section 4.1 .

\subsubsection{Landward Distance and Seaward Distance Parameters}

The "Landward Distance" input is the distance in meters that the cross-shore transect is projected landward from the offshore baseline (fig. 10), while the "Seaward Distance" input is the distance the cross-shore transect is projected seaward from the baseline. Together, the parameters represent the cross-shore transect footprint, which determines where feature delineation occurs and influences the angle of the chord line generated on each transect. Delineation can suffer when the chord line slope is more gradual than the foreshore slope (fig. 4), if the seaward extent of the transect falls on, or landward of, the true cliff toe or talus (section 2.4), or if the landward transect extent does not reach far enough landward of the cliff toe.

The landward transect distance can often be 95 percent of the total transect length. In most situations, the seaward distance may be kept short $(1 \mathrm{~m})$ but can also be extended to prevent the seaward end of the transect from falling on top of the cliff toe's true location when baselines 
intersect with the cliff face or true toe. (section 2.4). The seaward and landward distances should never extend cross-shore transects into areas of a DEM with null values, as this introduces error into feature delineation results. It is important to remember that if there is insufficient DEM coverage seaward of the baseline, the user can use ArcMap's "Euclidean Allocation" and "Mosaic to New Raster" tools to extend the DEM with proxy values drawn from the DEM's edge. ${ }^{12}$

TIP: A useful strategy for processing in this version of the CFDT is to generate cross-shore transects with a seaward extent that is set back from the seaward extent of the cliff face by a distance approximate to the specified spatial kernel size in meters. In this scenario, the refined delineation methods are likely to classify the cliff toe accurately, even when the basic toe is erroneous. The Baseline Builder Tool can be set up to build a baseline close to the cliff face, and the seaward transect distance can add seaward extent as needed. The "Landward Transect Distance" input should be set so that the true cliff top is easily encompassed by the cross-shore transect. In delineation tests on cliff features 20-50 $\mathrm{m}$ tall and without complex high-relief features landward of the cliff face, cliff toe and top delineation were still successful when the landward transect extent overshot the true cliff top by $40 \mathrm{~m}$ or more. There is little consequence, other than additional processing time, when the landward transect extent overshoots the true cliff top; if it falls short, the feature is erroneously classified.

\subsubsection{Transect Interval Parameter}

The "Transect Interval" input is the distance, in meters, along the offshore baseline between generated transects (fig. 10). This input determines the alongshore resolution of cliff feature outputs. A higher transect frequency (1-5 $\mathrm{m}$ or less) can be chosen when input DEMs have high resolution, cliffs are not very high relief, and study areas are small (1-km shoreline length or less) where fine scale linear outputs are meaningful. For larger regional analysis, alongshore transect frequency can be lower (5-50 $\mathrm{m}$ or more) to improve processing time. Increasing the frequency of transect generation increases processing time.

\subsubsection{Delineation Mode Parameter}

The Delineation Mode input determines which algorithms are used to identify final cliff features. The different mode options are described below:

- Basic Delineation: This setting outputs cliff tops, midpoints, and toes associated only with "Basic Delineation" (see section 2.3 for more information on how "Basic Delineation" works).

- Refined Delineation: This setting outputs cliff tops and midpoints as with "Basic Delineation," but cliff toes are derived from a chosen refined delineation method. The Refinement Method (either high pass or first concavity) is specified by the "Refinement Method" parameter below.

- Multi Delineation: This setting outputs cliff tops, midpoints, as well as all possible versions of refined toes. Outputs include a basic cliff toe, a cliff toe refined with the HighPass method, and a cliff toe refined with the First Concavity method.

\footnotetext{
${ }^{12}$ For more information on the Esri ArcGIS "Euclidean Allocation" tool, visit https://desktop. arcgis.com/en/arcmap/10.6/tools/spatial-analyst-toolbox/euclidean-allocation.htm. For more information on the Esri ArcGIS “Mosaic to New Raster” tool, visit https://desktop.arcgis.com/en/ arcmap/10.6/tools/data-management-toolbox/mosaic-to-new-raster.htm.
} 
TIP: "Basic Delineation" mode performs especially well on high-relief or sheer cliffs where cross-shore transects can be kept relatively short. Refined delineation methods perform comparatively well when the cliff morphology is complex and cross-shore transects must be extended.

\subsubsection{Refinement Method Parameter}

If "Refined Delineation" mode is selected in the previous step, there are two methods available for toe-position refinement:

- High Pass: This method identifies the cliff toe by analyzing a high-pass profile derived from the slope of the raster DEM underlying the cross-shore transects (see section 2.4 for more information on the "High Pass" method). This method of toe refinement is more likely to identify the toe at the seaward edge of a talus. Cliff tops and midlines are identified using the same method as "Basic Delineation" mode

- First Concavity: This method identifies the cliff toe by projecting a chord line between the coarsely delineated toe and the cliff midline, and then runs a one-dimensional slopeanalysis kernel across the elevation profile surface between the toe and midline (see section 2.4 for more information on how the first concavity method works). The size of the analysis kernel is determined by the Kernel Size input parameter further detailed in section 5.2.11.

TIP: "Multi Delineation" mode (section 5.2.7) generates high pass, first concavity, and basic toes. If the user is unsure which refinement method might yield the best results, "Multi Delineation" mode is an effective choice that negates the input for the "Refinement Method."

\subsubsection{High Pass Threshold Parameter}

The High Pass Threshold input controls the high-pass-filtered threshold used to refine the location of the cliff toe for the "High Pass" refined delineation method. A point along a transect with a greater negative value (further from zero) than the threshold triggers toe identification. If no value is triggered by the user-defined threshold on the lower half of the cliff, the value is automatically revised closer to zero until a threshold is triggered (see section 2.4 for more information on how the "High Pass" method works).

The default value of -25 may not be optimal for all cliff morphologies, but it is an effective starting point. First running CFDT with the default value of -25 and evaluating the delineated features in a 3D format is recommended, as high-pass values interpreted without context could appear arbitrary. If the cliff toe is consistently delineated landward of the true toe location, consider using a value closer to zero. If the delineated location is consistently seaward of the true toe location, consider a value further from zero. The allowed input range is from -6 to -50 .

TIP: If the user chooses "First Concavity" for the "Refinement Method" parameter, this input is negated.

\subsubsection{Subsidiary Feature Delineation Parameter}

The Subsidiary Feature Delineation input determines whether subsidiary features are delineated as 3D point shapefiles. Subsidiary features are convexities and concavities on the cliff face and can represent geologic controls on cliff morphology (see section 2.5 for more information on how "Subsidiary Feature Delineation" works). 
- Yes: Subsidiary features are delineated; this is the default setting. The algorithm allows one convexity and one concavity to be delineated on the lower and upper halves of the cliff, permitting a maximum of four points per cross-shore transect.

- No: Subsidiary features are NOT delineated.

Like "First Concavity" toe refinement, "Subsidiary Feature Delineation" uses a one-dimensional slope-analysis kernel, and the size of the one-dimensional kernel used in this process is enforced by the "Kernel Size" parameter in section 5.2.11.

\subsubsection{Kernel Size Parameter}

The "Kernel Size" input modifies the size of the one-dimensional analysis kernel used in "First Concavity" toe refinement and "Subsidiary Feature Delineation." The kernel size is the number of cells in a processing kernel (the kernel's neighborhood) and the default input is three cells (resulting in a $1 \times 3$ cell neighborhood running along the cross-shore transect). Because a kernel must have a central member, the kernel size must be an odd value and can never be less than three cells in size. Users are encouraged to check the spatial resolution of their input DEM when choosing a kernel size so that they are aware of the planar length of the kernel in meters.

During tool development, subsidiary delineation was tested on a 1-m resolution DEM with kernel sizes of three and five cells (3-m and 5-m spatial kernels). Both kernel sizes yielded acceptable concavity locations on cliffs $30-40 \mathrm{~m}$ in height. If higher resolution DEMs are used as inputs, users should consider increasing the kernel size to maintain a relevant spatial kernel length. For instance, for a DEM with a cell resolution of $0.5 \mathrm{~m}$, a kernel size of seven would result in a 3.5-m spatial kernel. Significantly smaller cliffs benefit from a smaller spatial kernel, and taller or gradually sloping cliffs benefit from a larger spatial kernel. When all other conditions are equal, a larger spatial kernel is less sensitive to deviations in surface slope over small distances.

\subsubsection{Output Smoothing Tolerance Parameter}

The Output Smoothing Tolerance input (in meters) determines the level of smoothing performed on cliff feature line outputs. The higher the tolerance, the more smoothing is performed. A lower tolerance results in fewer modifications to output line features while leaving the feature with more angular transitions between segments.

\subsubsection{Output Cleanup Parameter}

The Output Cleanup input determines how to handle feature-line cutbacks or regions where more than one instance is identified along a single cross-section profile. Cutbacks can occur along crenulated sections of coast and narrow headlands where offshore baselines have excessive curvature (fig. 12). Cutbacks can also be identified in areas where cross-shore transects overlap with both sides of a jutting headland. Cutbacks can be handled in two ways:

- Flag Cutbacks: This is the default setting. The tool flags cross-shore transects with cutbacks along their extent, saving them for use in data review and modification by the user. This setting is also useful in situations where the user does not want to automatically delete the outputs around narrow, jutting headlands.

- Delete Cutbacks: The tool automatically deletes sections of the cliff feature lines around cross-shore transects on which cutbacks were detected. 


\subsubsection{Series Name Parameter}

The Series Name input adds a user-specified suffix to the names of final model outputs. Final outputs are written to the "Data" folder within the workspace. The closed phrase "SeriesName" in the following shapefile output conventions is merely a placeholder for the name a user assigns when generating final model output files. The naming conventions for outputs are as follows:

- Cliff top polyline shapefile: CliffTopLine3D_SeriesName.shp

- Cliff midline polyline shapefile: Midline3D_SeriesName.shp

- Basic cliff toe polyline shapefile: CliffToeLine3D_SeriesName.shp

- Cross-shore transects polyline shapefile: Transects3D_SeriesName.shp

- Refined toe polyline shapefile (High Pass): RefinedToeHPLine3D_SeriesName.shp

- Refined toe polyline shapefile (First Concavity): RefinedToeFCLine3D_SeriesName.shp

- Subsidiary features point shapefile: SubsidiaryFeatures3D_SeriesName.shp

- Basic toe, refined toes, midpoints, and tops point shapefile: PrimaryFeaturePoints SeriesName.shp

- Flagged cutback transects: CutbackTransects_SeriesName.shp

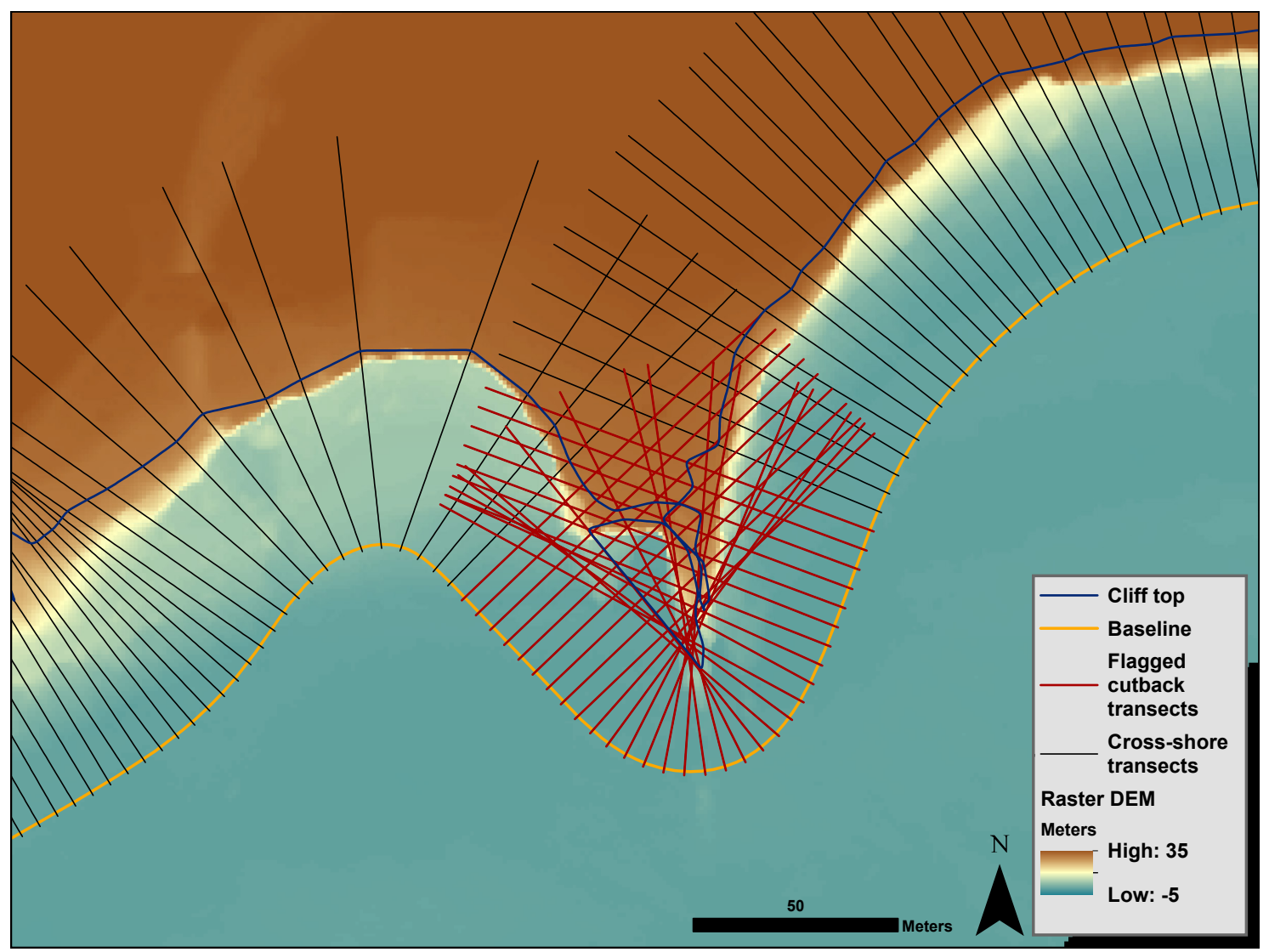

Figure 12. An image showing the cliff top line, cross-shore transects, and flagged cutback transects. Cutback transects mark any locations where cliff feature lines, such as the cliff top, cut back on themselves alongshore. In this example, feature line cutbacks occurred because the baseline exhibited too much curvature around a jutting headland. DEM, digital elevation model. 
Additionally, an error-log table is generated in the "Tables" folder within the workspace following the naming convention TransectErrorRecord_SeriesName.csv. If there are unexpected gaps in the delineated outputs, they may be explained in this table.

\section{Using the Baseline Builder Tool and Vectorizing an Offshore Baseline}

\subsection{Baseline Builder Tool Overview}

As mentioned in section 5.2.1, offshore baselines should be digitized close to the cliff features delineated (approximately 5-30 pixels seaward of the cliff face). By using refined delineation methods, baselines can be farther from the cliff and still produce clear feature delineations. However, initiating cross-shore transects farther from the cliff increases the probability that adjacent cross-shore transect lines cross over one another before reaching the cliff face, generating cliff feature cutbacks during delineation. Consistent baseline placement is also useful when digital surface models from photogrammetric approaches are used as "Raster DEM" inputs into the CFDT, as these datasets rarely extend seaward of the subaerial beach and cannot accommodate cross-shore transects cast from offshore where no data exist.

Maintaining a consistent distance from the cliff face and line curvature when manually vectorizing the offshore baseline from a DEM or aerial image can be difficult. To this end, a Baseline Builder Tool is included in the working directory toolbox that can generate an automated baseline around cliff features in a study area. Users can treat this baseline as the "Offshore Baseline" input into CFDT or use it as a manual vectorization guide, helping users vectorize their baseline at consistent distances from cliff features.

The Baseline Builder Tool operates by scanning the DEM and selecting areas where high relief features meet the topographic relief threshold specified by the user. First, an analysis kernel calculates the elevation range in a neighborhood around each pixel of the DEM, with the results output to a new raster (figs. $13 A, B$ ). Cells that meet or exceed an elevation-range threshold specified by the user are then classified as relief regions (fig. 13C). Both the size of the kernel neighborhood and the elevation-range threshold are modifiable, allowing the user to direct the algorithm to only classify certain cliff morphologies. For instance, by specifying a relatively small kernel neighborhood and a relatively large elevation-range threshold, only tall, high-relief cliffs classify as relief regions. The classified area is then buffered by the distance the user chooses the offshore baseline to be from the cliff face (fig. 13D).

The above topographic analysis scans the entire input DEM for relief regions, and buffers are produced both landward and seaward of classified pixels (fig. 13D). Segments are filtered by their maximum elevation to remove buffer segments occurring on the landward side of cliffs (fig. 13E). Specifically, the filter removes buffer segments with a maximum elevation above the mean elevation of the classified relief area, minus 1 standard deviation. Finally, the remaining buffer is smoothed with a tolerance specified by the user (fig. 13F). This process leaves smoothed polylines offset a consistent distance seaward of classified relief features. The user then enters a short editing session where the smoothed line is quality-reviewed for use as an offshore baseline input or used as a vectorization guide for a manually digitized baseline. 


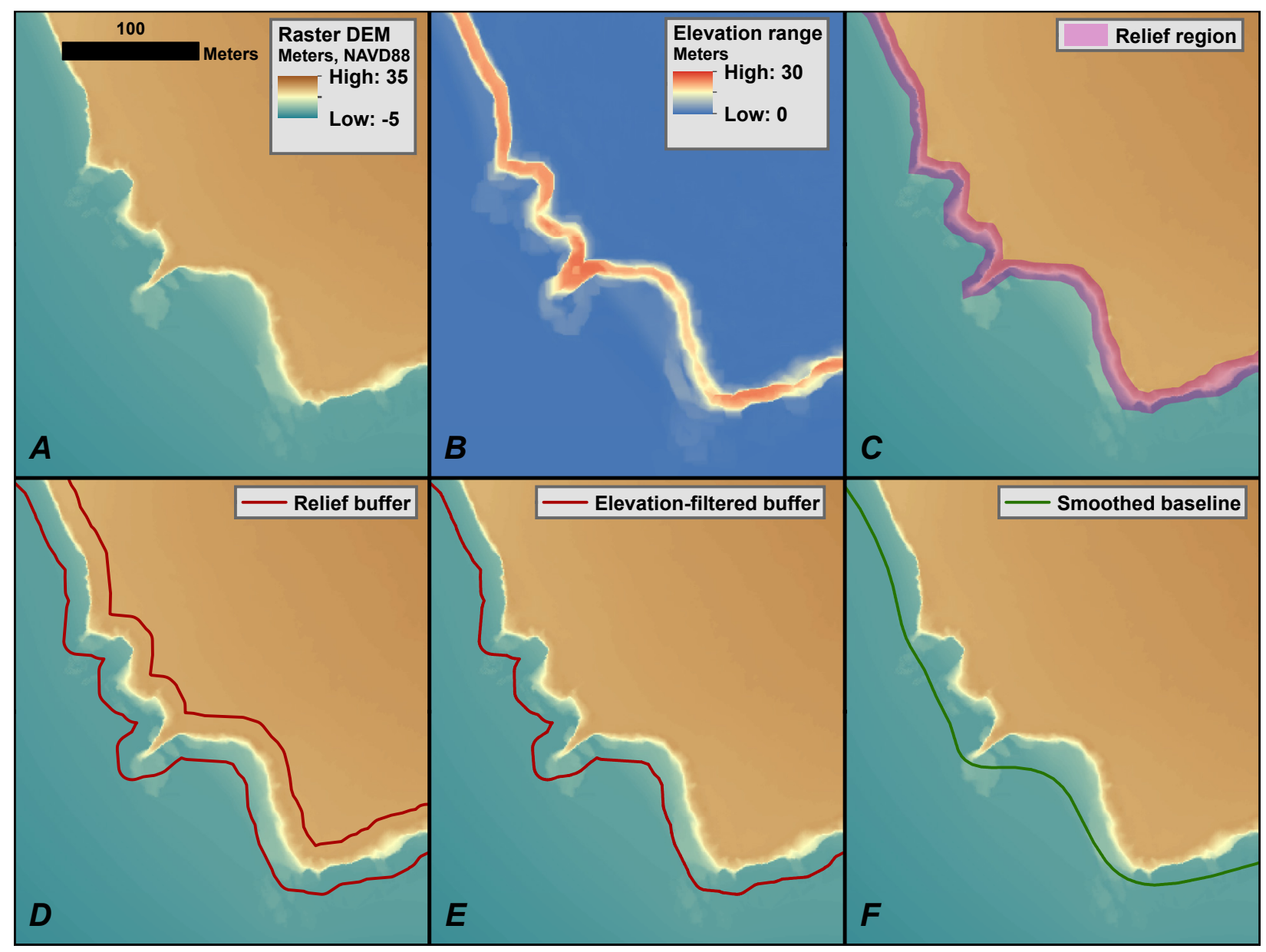

Figure 13. Images showing $(A)$ the "Raster DEM" input into the Baseline Builder Tool; $(B)$ the elevation range around each pixel within the neighborhood of the analysis kernel; $(C)$ a classified relief region satisfying the user's elevation range specifications; $(D)$ a polygon buffer projected outwards around the classified relief region; $(E)$ the buffer remaining after being elevation-filtered to remove any landward components; and $(F)$ the final, smoothed offshore baseline. DEM, digital elevation model; NAVD 88, North American Vertical Datum of 1988. 


\subsection{Running the Baseline Builder Tool}

Follow the steps in figure 14 to operate the Baseline Builder Tool.

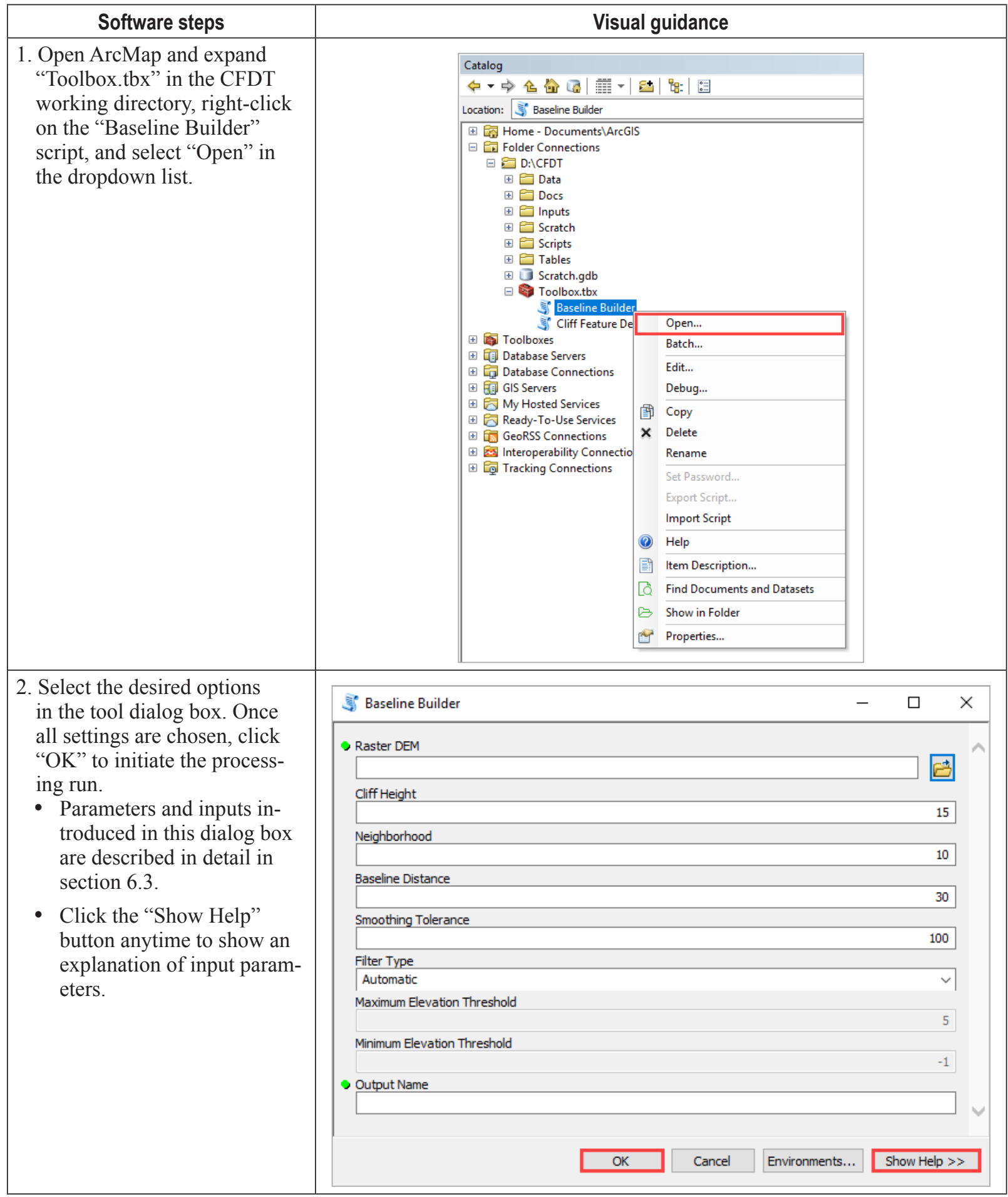

Figure 14. Instructions for running the Baseline Builder Tool. CFDT, Cliff Feature Delineation Tool; DEM, digital elevation model. 


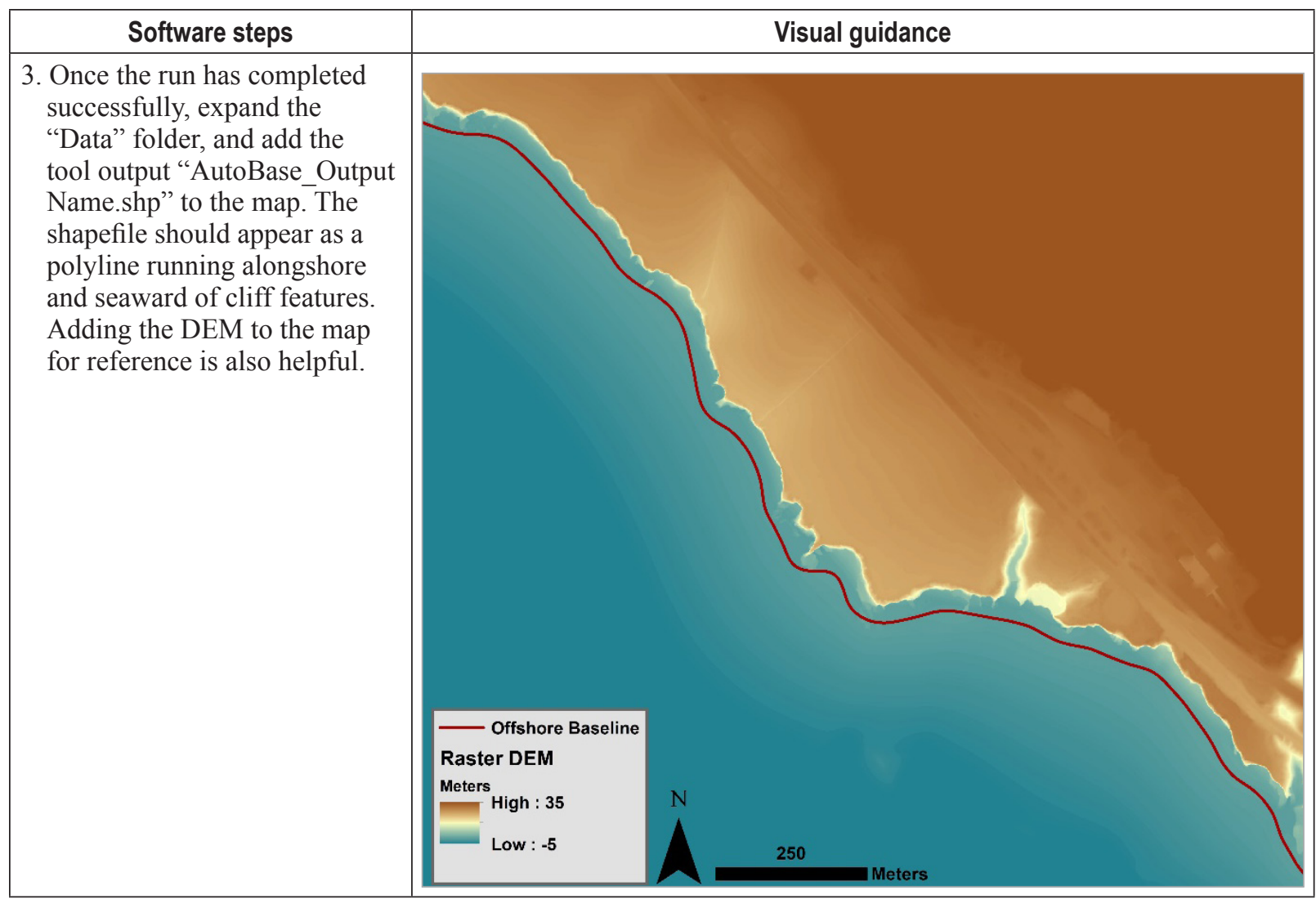

Figure 14. Instructions for running the Baseline Builder Tool. CFDT, Cliff Feature Delineation Tool; DEM, digital elevation model.-Continued

TIP: Like the CFDT, the Baseline Builder Tool generates intermediate outputs in the "Scratch" folder, which are overwritten during each processing run. One output in particular, "CliffRegion.tif," shows the raster areas the Baseline Builder Tool classified as meeting the user's "Cliff Height" and "Neighborhood" settings. If unsatisfactory gaps are seen in the baseline output in step 3, they should correspond to gaps in this intermediate output where relief was too low to satisfy the input parameters. The user can then rerun the Baseline Builder Tool with more appropriate "Cliff Height" and "Neighborhood" settings or digitize that section manually.

\subsection{Explanation of Baseline Builder Tool Starting Parameters}

Explanations of the Baseline Builder Tool inputs and starting parameters introduced in figure 14, step 2, are given in this section.

\subsubsection{Input DEM Description}

The Input DEM is the DEM containing the cliff features from which the baseline should be built seaward. This is the same "Raster DEM" that is used for cliff feature delineation. 


\subsubsection{Cliff Height Setting}

The Cliff Height is the minimum height of cliffs, in meters, the user desires to delineate in the study area. Selecting a greater height prevents a baseline from being built offshore from lower cliffs. This height should be interpreted as the vertical distance between the beach or cliff toe and the top of the cliff.

\subsubsection{Neighborhood Setting}

The Neighborhood is the 2D kernel size, in meters, used to scan the DEM for the userspecified "Cliff Height." If the elevation range over the areas of the kernel meets or exceeds the parameter for "Cliff Height," the central kernel member is classified as cliff area, and a baseline can be built seaward of it.

\subsubsection{Baseline Distance Input}

The Baseline Distance is the chosen seaward distance, in meters, between the cliff face and the offshore baseline. It determines how close the baseline or digitizing guide is built from the cliff.

\subsubsection{Smoothing Tolerance Input}

The Smoothing Tolerance input controls how smooth the curvature is on output baselines. For this application, a minimum "Smoothing Tolerance" of 100-150 $\mathrm{m}$ is necessary to remove sharp bends in the baseline. The higher the "Smoothing Tolerance," the more gradual the curvature in the output baseline (fig. 15).

\subsubsection{Filter Type Parameter}

The Baseline Builder Tool constructs polylines on both landward and seaward sides of relief features and uses elevation filtering to extract only the polylines seaward of relief features. Two modes of elevation filtering for the Filter Type input can be used: "Automatic" and "Manual Threshold."

- Automatic: The "Automatic" setting works well when the input raster DEM does not extend far inland or when most relief features detected by the "Cliff Height" and "Neighborhood" parameters are associated with the seacliffs. This input requires no additional input from the user. The performance under this setting degrades when rasters extend further inland and more relief features are classified at higher elevations.

- Manual Threshold: The "Manual Threshold" setting works well when the raster DEM extends far inland or when many relief features are classified away from the shoreline at higher elevations. When enabled, the algorithm extracts polylines falling within a userspecified elevation range (see the "Maximum" and "Minimum Elevation Threshold" parameters below).

\subsubsection{Maximum Elevation Threshold Setting}

Polylines occurring above the Maximum Elevation Threshold elevation are filtered out of baseline results under the "Manual Threshold" filter-type setting; this setting is unavailable under the "Automatic" filter-type setting. 


\section{Using the Baseline Builder Tool and Vectorizing an Offshore Baseline}

\subsubsection{Minimum Elevation Threshold Setting}

Polylines occurring below the Minimum Elevation Threshold elevation are filtered out of baseline results under the "Manual Threshold" filter-type setting; this setting is unavailable under the "Automatic" filter-type setting.

\subsubsection{Output Name}

The Output Name is the suffix of the name given to the output digitization guide or automatic baseline: AutoBase_OutputName.shp.

TIP: The "Cliff Height" and "Neighborhood" parameters can be interpreted as scanning for levels of topographic relief and slope. Thus, the user can specify heights and kernel areas that are associated with features much shorter than cliffs and that occur over shorter planar distances.

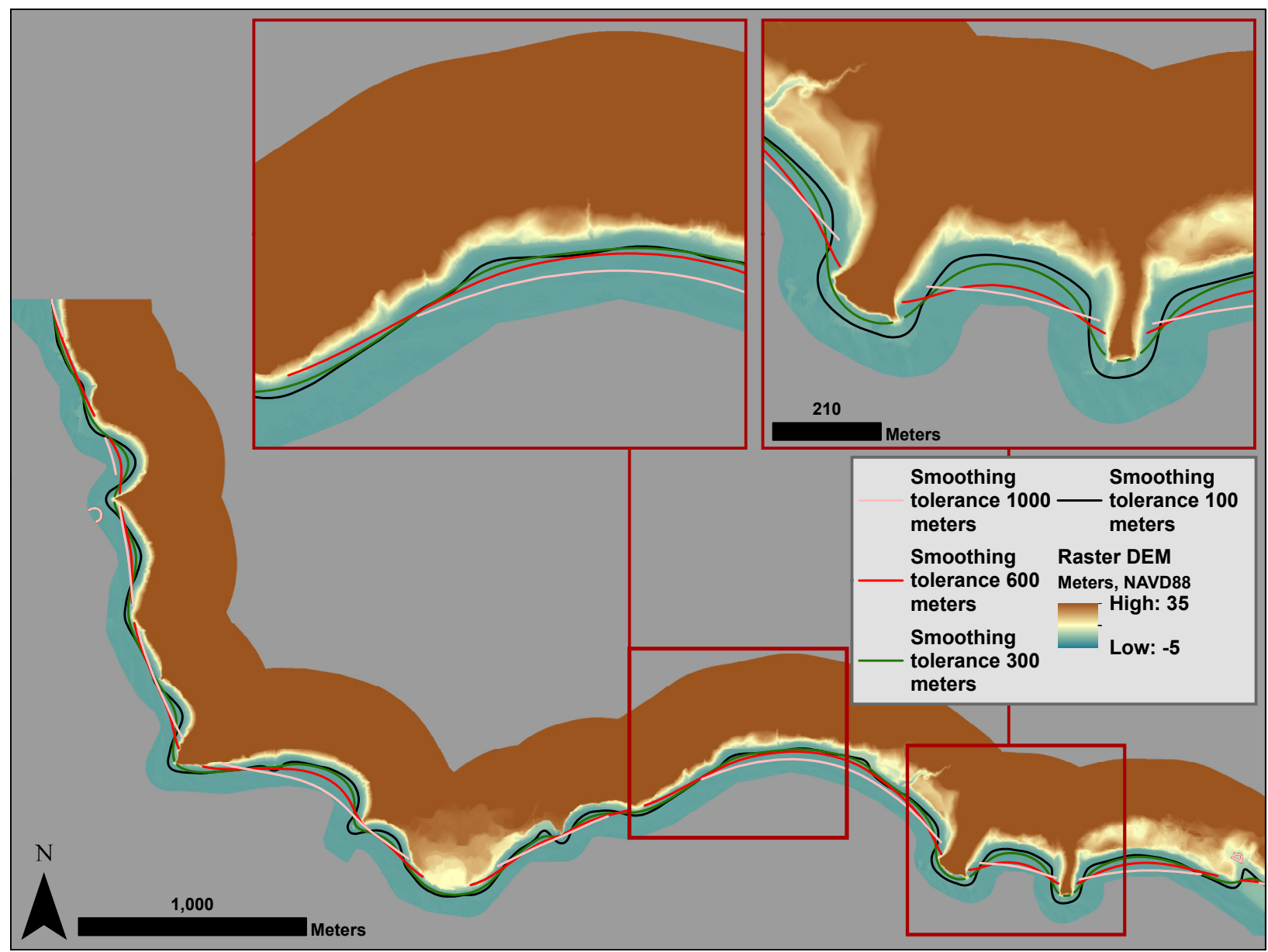

Figure 15. An image showing offshore baselines generated with different smoothing tolerances (1,000 meters [m], $600 \mathrm{~m}, 300 \mathrm{~m}$, and $100 \mathrm{~m})$ using the Baseline Builder Tool. Cross-shore transects are projected perpendicular to a given baseline segment, so the smoothing tolerance increases or decreases the local variability in the bearing of the transects cast. If aggressive smoothing brings the baseline in contact with cliff features, the baseline is automatically clipped. DEM, digital elevation model; NAVD 88, North American Vertical Datum of 1988. 


\subsection{Reviewing Baseline Builder Tool Outputs}

Follow the steps in figure 16 to edit the Baseline Builder Tool output for use as an offshore baseline.

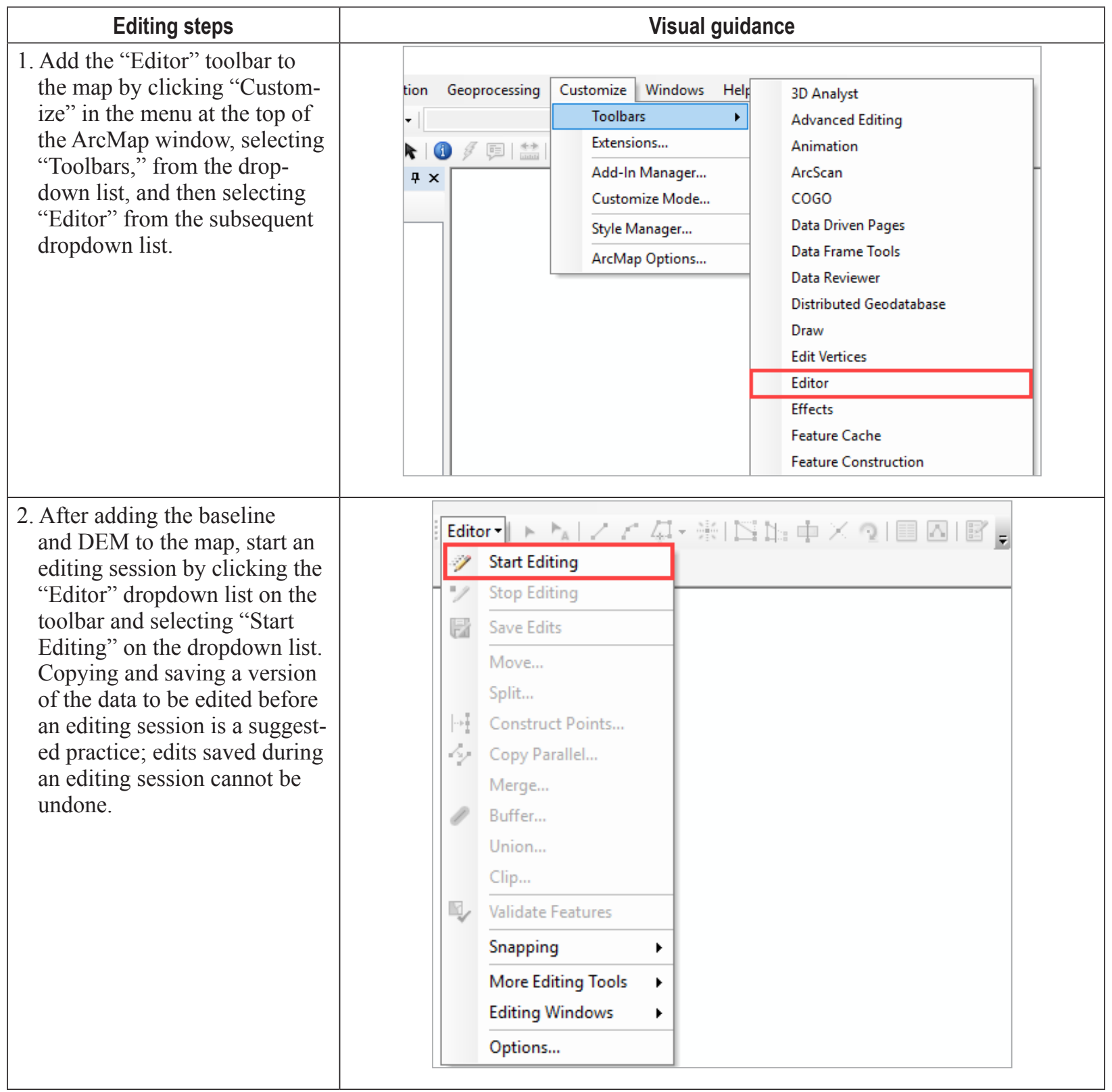

Figure 16. Instructions for editing the Baseline Builder Tool output for use as an offshore baseline. 


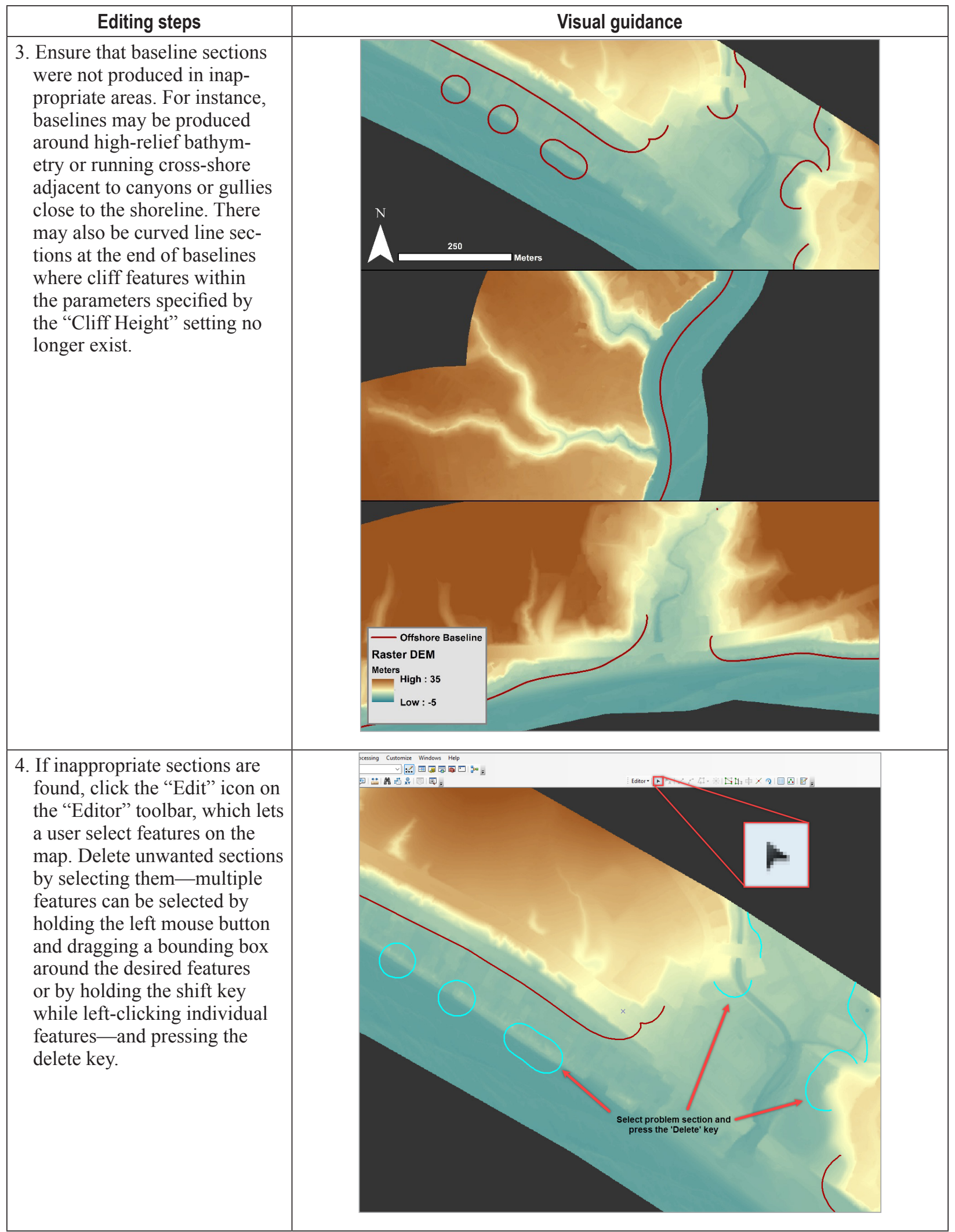

Figure 16. Instructions for editing the Baseline Builder Tool output for use as an offshore baseline.-Continued 


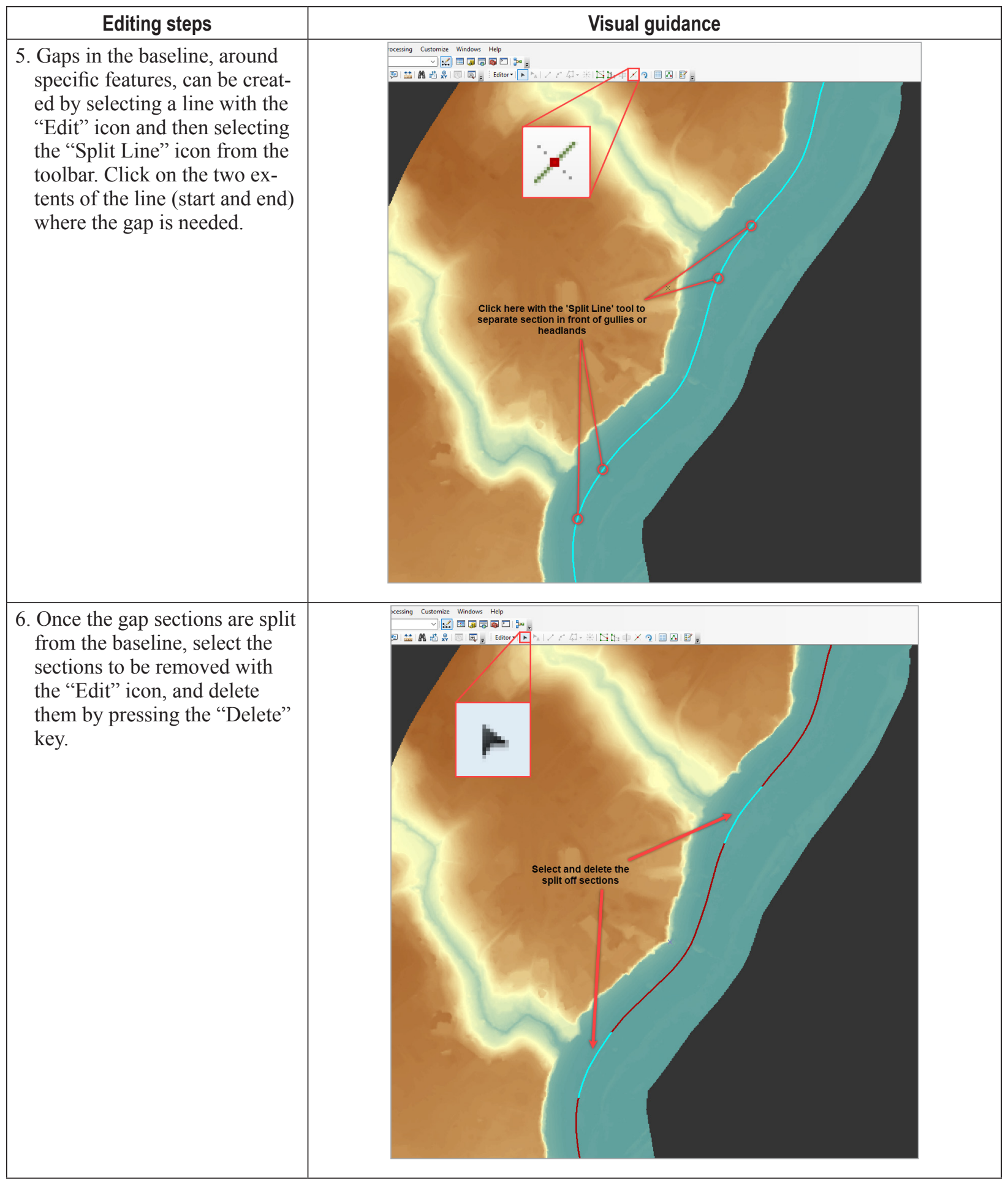

Figure 16. Instructions for editing the Baseline Builder Tool output for use as an offshore baseline.-Continued 


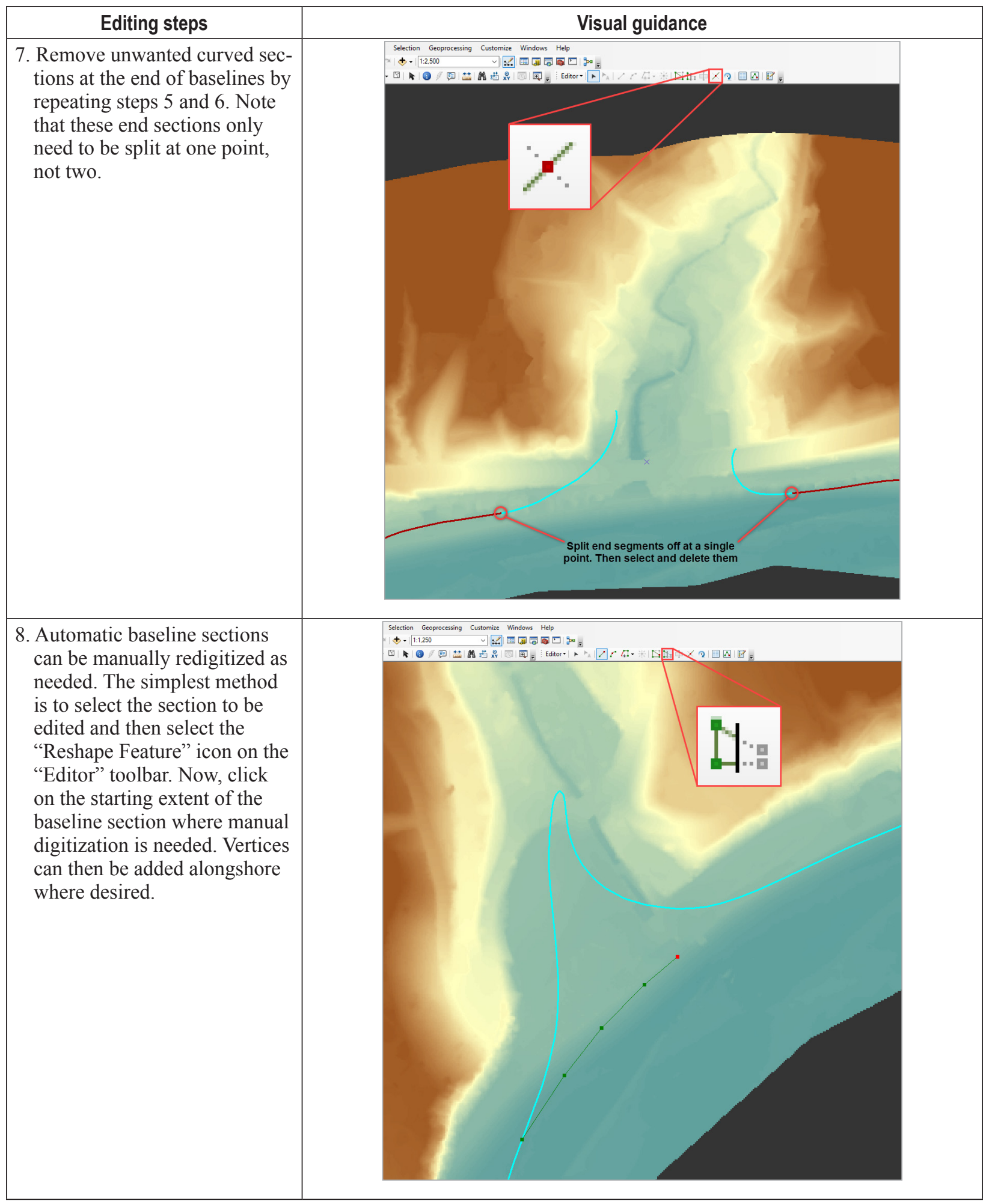

Figure 16. Instructions for editing the Baseline Builder Tool output for use as an offshore baseline.-Continued 


\begin{tabular}{|c|c|}
\hline Editing steps & Vis \\
\hline $\begin{array}{l}\text { 9. The final vertex must end on } \\
\text { the automatically generated } \\
\text { baseline, representing the end } \\
\text { extent of the section to be } \\
\text { reshaped. Right-click and se- } \\
\text { lect "Finish Sketch" from the } \\
\text { dropdown list that appears. }\end{array}$ & 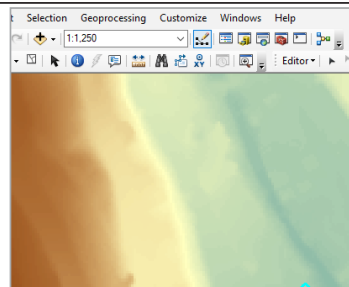 \\
\hline \multirow{9}{*}{$\begin{array}{l}\text { 10. Once all changes are made, } \\
\text { expand the "Editor" dropdown } \\
\text { list and click "Save Edits." } \\
\text { Next, click "Stop Editing." } \\
\text { The baseline can now be used } \\
\text { as an input to the CFDT. }\end{array}$} & \multirow{2}{*}{ 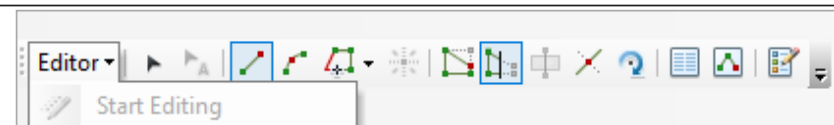 } \\
\hline & \\
\hline & - Stop Editing \\
\hline & Save Edits \\
\hline & \multirow[t]{2}{*}{ 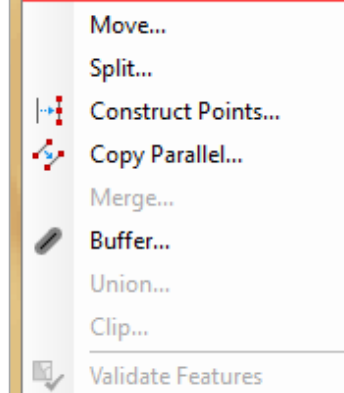 } \\
\hline & \\
\hline & Snapping \\
\hline & $\begin{array}{l}\text { More Editing Tools , } \\
\text { Editing Windows }\end{array}$ \\
\hline & Options... \\
\hline
\end{tabular}

Figure 16. Instructions for editing the Baseline Builder Tool output for use as an offshore baseline.-Continued

TIP: Steps 4-7 require that the "Snapping" setting be enabled, and an appropriate snapping tolerance can make baseline editing easier. To enable or modify the snapping tolerance, open the "Editor" toolbar dropdown list, navigate to "Snapping," click "Options," and set the "Tolerance." A higher number causes the mouse cursor to snap to the baseline from farther away, and a tolerance of 0 disables snapping. 


\section{Using the Baseline Builder Tool and Vectorizing an Offshore Baseline}

\subsection{Manually Digitizing an Offshore Baseline}

Follow the steps in figure 17 to manually digitize a baseline using the Baseline Builder Tool output as a guide.

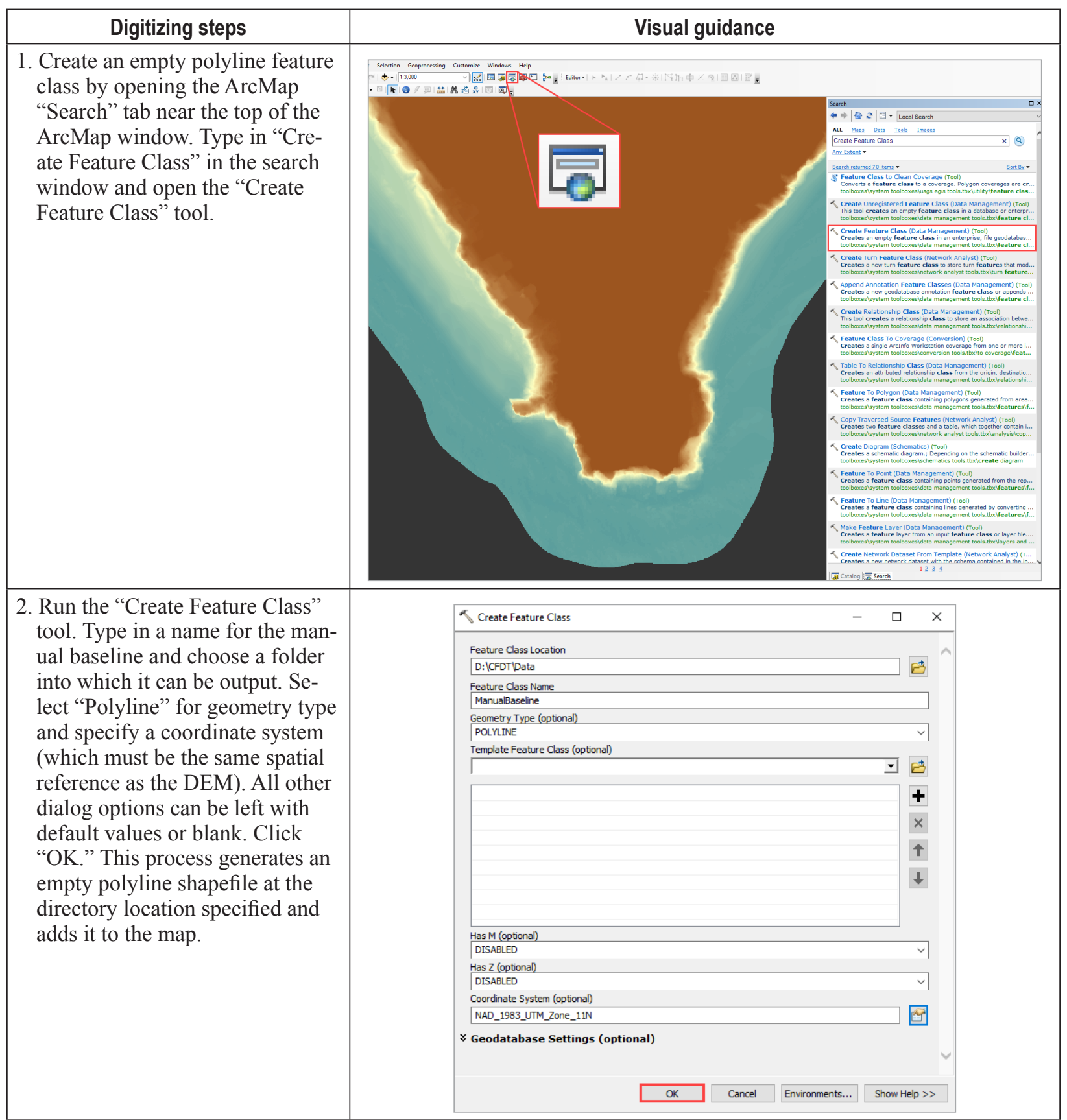

Figure 17. Instructions for manually digitizing a baseline, with the Baseline Builder Tool output used as a digitizing guide. CFDT, Cliff Feature Delineation Tool; DEM, digital elevation model. 


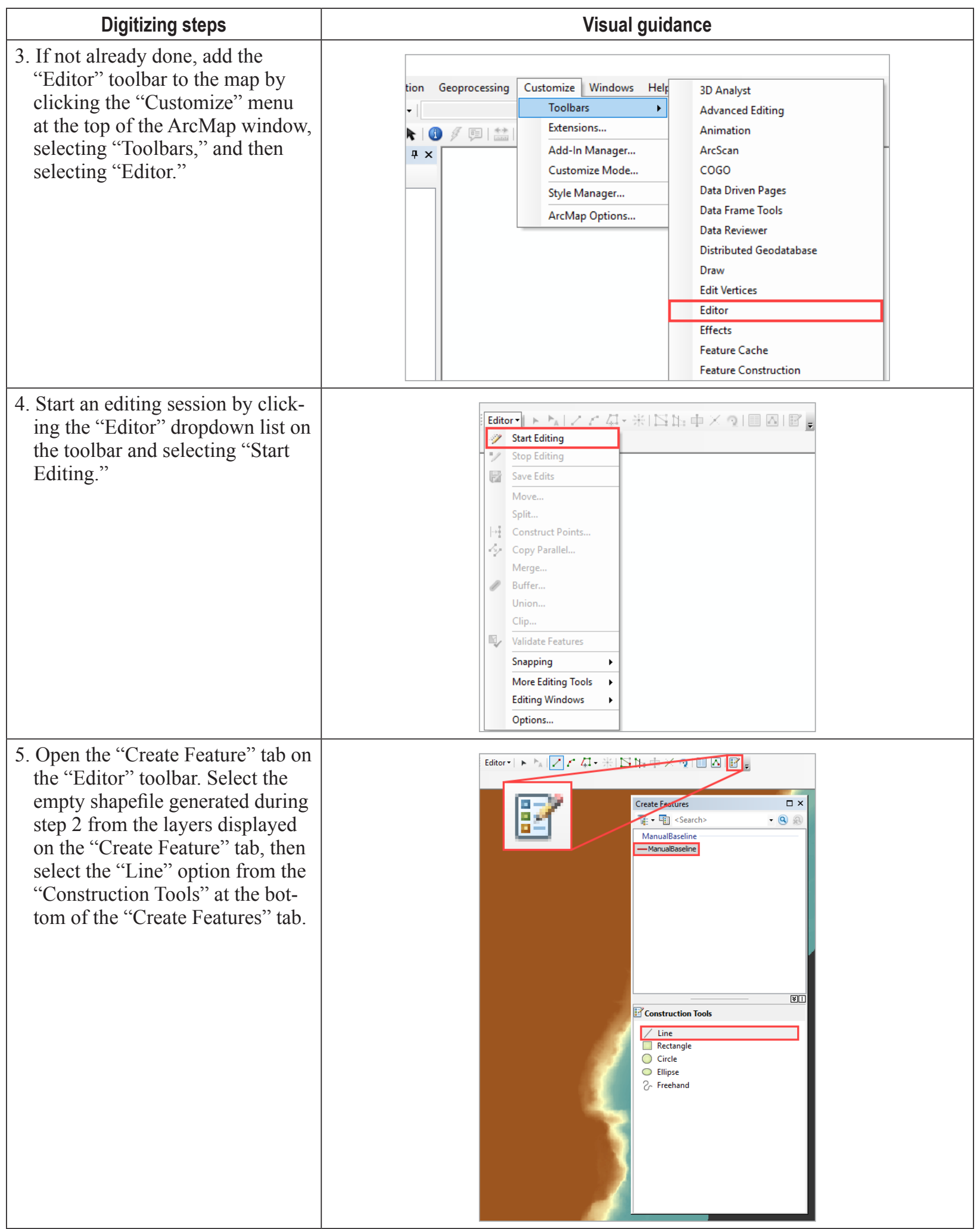

Figure 17. Instructions for manually digitizing a baseline, with the Baseline Builder Tool output used as a digitizing guide. CFDT, Cliff Feature Delineation Tool; DEM, digital elevation model.—Continued 


\section{Using the Baseline Builder Tool and Vectorizing an Offshore Baseline}

\begin{tabular}{|c|c|}
\hline Digitizing steps & Visual guidance \\
\hline $\begin{array}{l}\text { 6. Click on the map to manually } \\
\text { add baseline vertices. }\end{array}$ & 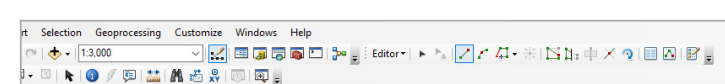 \\
\hline $\begin{array}{l}\text { 7. If needed, hovering the mouse } \\
\text { cursor near an automated base- } \\
\text { line added to the map snaps the } \\
\text { mouse cursor onto it. The snap- } \\
\text { ping tolerance can be changed } \\
\text { (making it easier or harder for } \\
\text { the cursor to snap to the baseline } \\
\text { extent) by opening the "Editor" } \\
\text { toolbar dropdown list, navigating } \\
\text { to "Snapping," selecting "Op- } \\
\text { tions," and changing the "Toler- } \\
\text { ance." A higher number causes } \\
\text { the input-device cursor to snap to } \\
\text { the baseline from farther away, } \\
\text { and a tolerance of zero disables } \\
\text { snapping. }\end{array}$ & 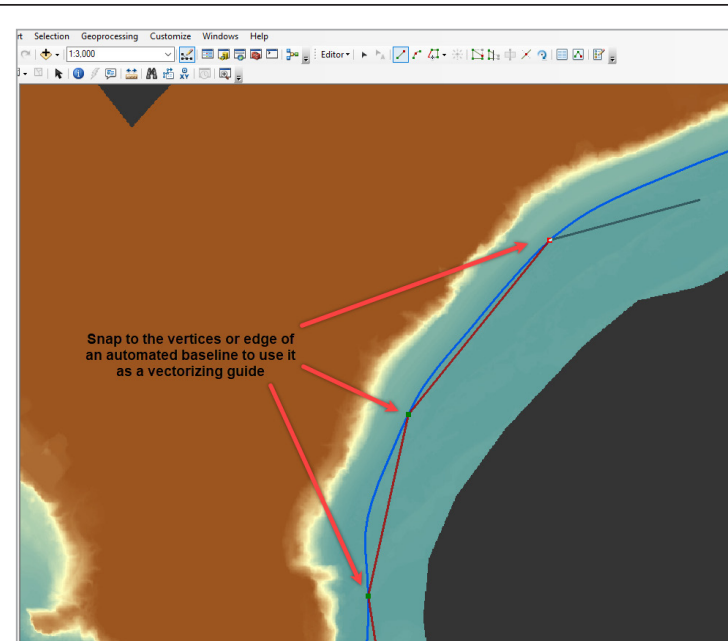 \\
\hline
\end{tabular}

Figure 17. Instructions for manually digitizing a baseline, with the Baseline Builder Tool output used as a digitizing guide. CFDT, Cliff Feature Delineation Tool; DEM, digital elevation model.—Continued 


\begin{tabular}{|c|c|c|}
\hline Digitizing steps & \multicolumn{2}{|c|}{ Visual guidance } \\
\hline $\begin{array}{l}\text { 8. Finish a baseline section at any } \\
\text { time by right-clicking on the map } \\
\text { and selecting "Finish Sketch." } \\
\text { Alternatively, cancel a baseline } \\
\text { segment by right-clicking and } \\
\text { selecting "Delete Sketch." Sub- } \\
\text { sequent sections can be added by } \\
\text { following steps 6-7 and clicking } \\
\text { "Finish Sketch" at the end of } \\
\text { each segment. }\end{array}$ & $/ 1$ & 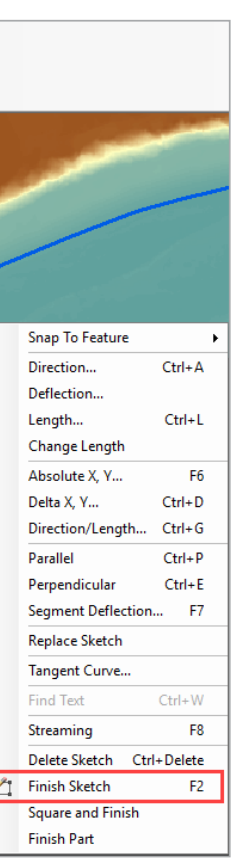 \\
\hline $\begin{array}{l}\text { 10. Once all segments are digitized, } \\
\text { expand the "Editor" menu and } \\
\text { click "Save Edits." Then click } \\
\text { "Stop Editing" to end the editing } \\
\text { session. The baseline is now } \\
\text { ready to be used in the CFDT. }\end{array}$ & \begin{tabular}{|l|l|} 
Editor & Start Editing \\
Stop Editing \\
Save Edits \\
Sove... \\
Split... \\
Construct Points... \\
Berge... \\
Buffer... \\
Clip.... \\
Validate Features \\
Snapping \\
More Editing Tools \\
Editing Windows \\
Options... \\
\end{tabular} & 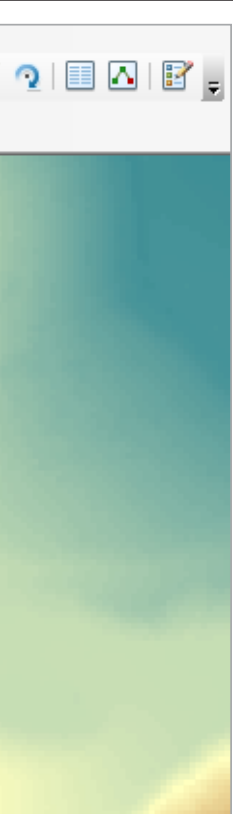 \\
\hline
\end{tabular}

Figure 17. Instructions for manually digitizing a baseline, with the Baseline Builder Tool output used as a digitizing guide. CFDT, Cliff Feature Delineation Tool; DEM, digital elevation model._Continued

\section{Visualizing and Reviewing Cliff Feature Delineation Tool Outputs}

Several visualization and review options are available for CFDT outputs. Features can be projected in a 3D format in ArcScene, which provides the most rapid means of assessing the validity of tool outputs and is the preferred format for manually editing cliff feature lines. Data products 
can also be displayed in a 2D format in ArcMap where cross-shore elevation profile graphs can be generated from transects. Both ArcScene and ArcMap give access to output shapefile attribute tables which provide coordinate information on the location of delineated features on cross-shore transects.

\subsection{Visualizing Features in ArcScene}

To open the CFDT outputs in a 3D environment, first open ArcScene, which should be included in the ArcGIS installation. Next, open the "Catalog" and, if necessary, connect to the CFDT working directory before expanding the "Data" folder, as described in section 3.2. Add any of the desired cliff feature outputs to the map by dragging the shapefiles from the catalog into the main map panel. A list of the possible outputs can be found in section 5.2.14.

To change the symbology for output shapefiles, navigate to the "Table of Contents" and double click on the current symbology for a given shapefile, which opens the "Symbol Selector" (fig. 18). Change the line or point color or width and click "OK" when finished.

Several navigation tools are available at the top of the ArcScene window to allow the user to explore the 3D scene. Ensure that the navigation tools are visible by opening the "Customize" menu, expanding "Toolbars," and ensuring "Tools" is checked. Use "Navigate Mode" to change the view of outputs in the map panel (fig. 19). Holding the left mouse button while moving the mouse rotates the current view. Scrolling the center mouse wheel zooms in and out of the current view. The scene can be panned by using "Pan Mode" (fig. 19) or pressing down the central mouse wheel while dragging the mouse. The level of zooming and panning permitted by ArcScene depends on how far the current view is panned from a "Camera Target," which is not overtly displayed to the user. If panning or zooming becomes restricted, set a new camera target on a feature in the center of the screen by clicking a desired feature with the "Center on Target" button (fig. 19) or by holding down the "Ctrl" key and left-clicking on a feature while in "Navigate Mode." Returning to a full view of all features in the map is accomplished by clicking on the "Full Extent" icon (fig. 19).

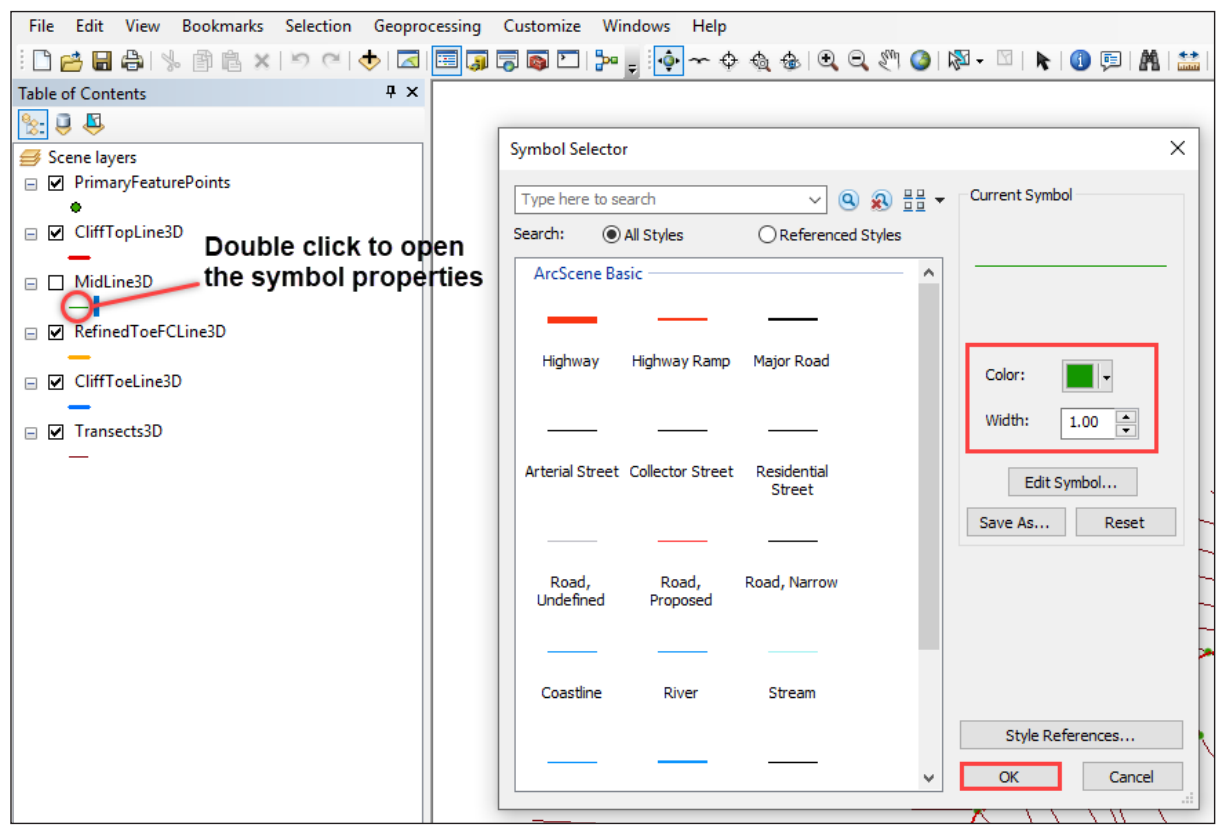

Figure 18. An image showing the ArcScene "Table of Contents" and "Symbol Selector," which can be used to change the symbology of output shapefiles. 


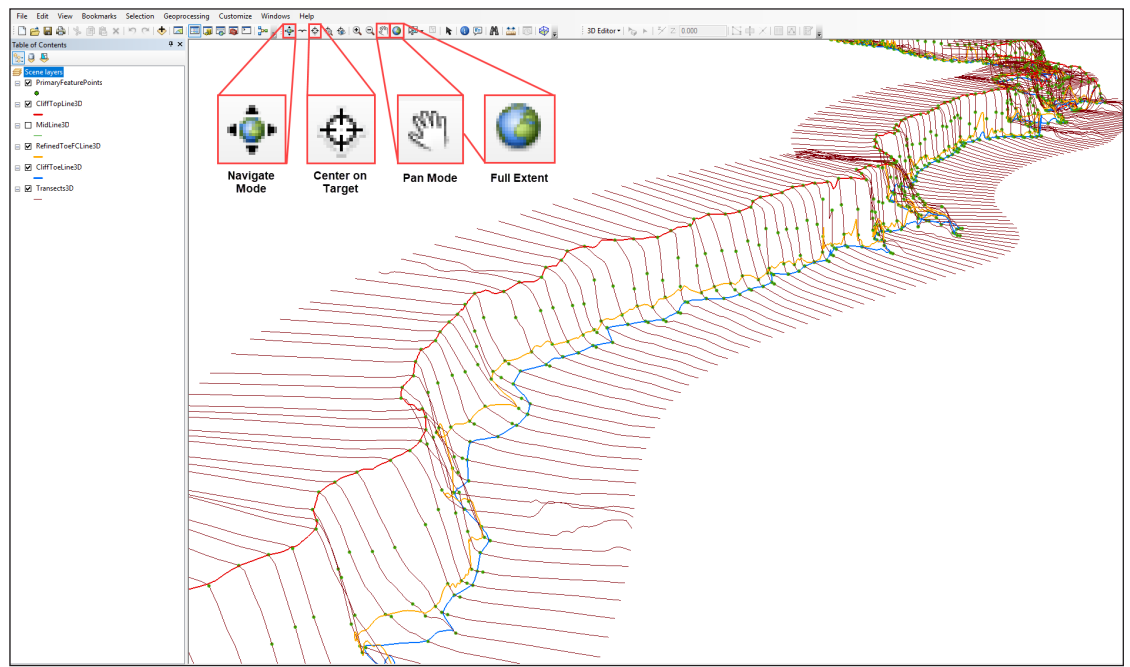

Figure 19. An image showing the key navigation tools (bracketed in red) for modifying the camera view in ArcScene, along with selected cliff feature outputs.

\subsection{Modifying Features in ArcScene}

The process for editing 3D features is like the process for digitizing offshore baselines outlined in section 5.2.1. Complete the steps in figure 20 to modify any cliff feature line.

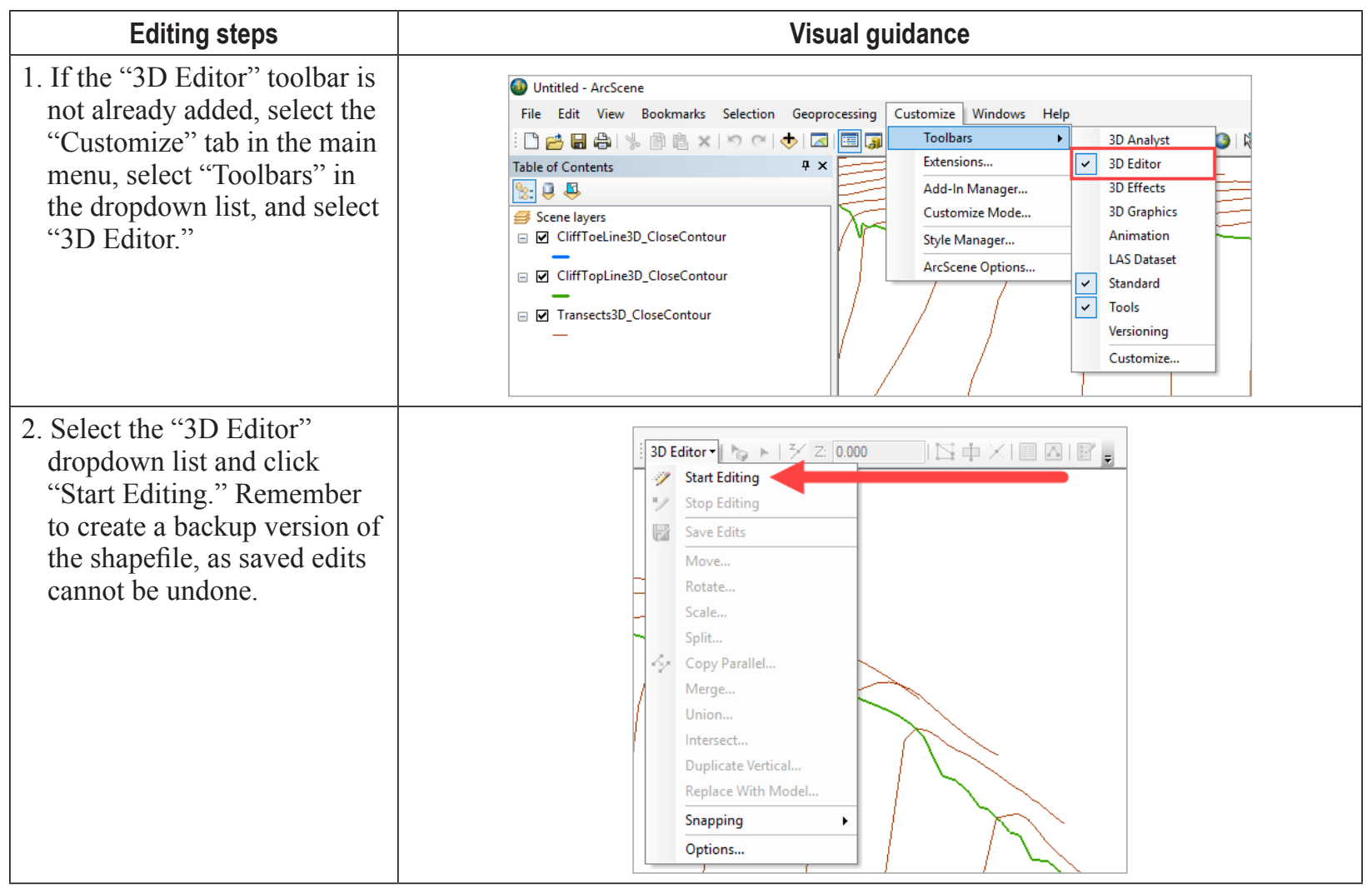

Figure 20. Instructions for editing any cliff feature line in ArcScene. 


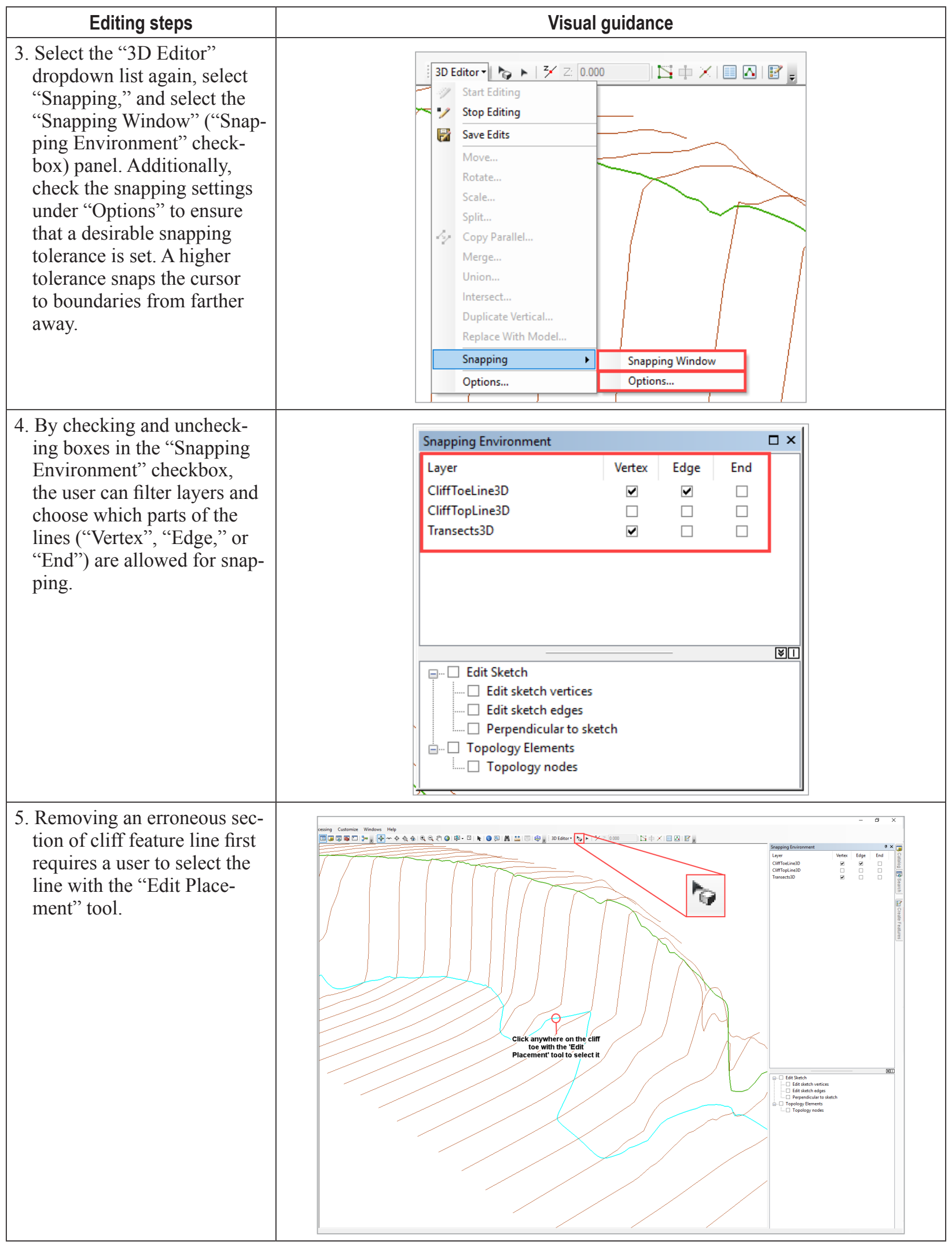

Figure 20. Instructions for editing any cliff feature line in ArcScene.-Continued 


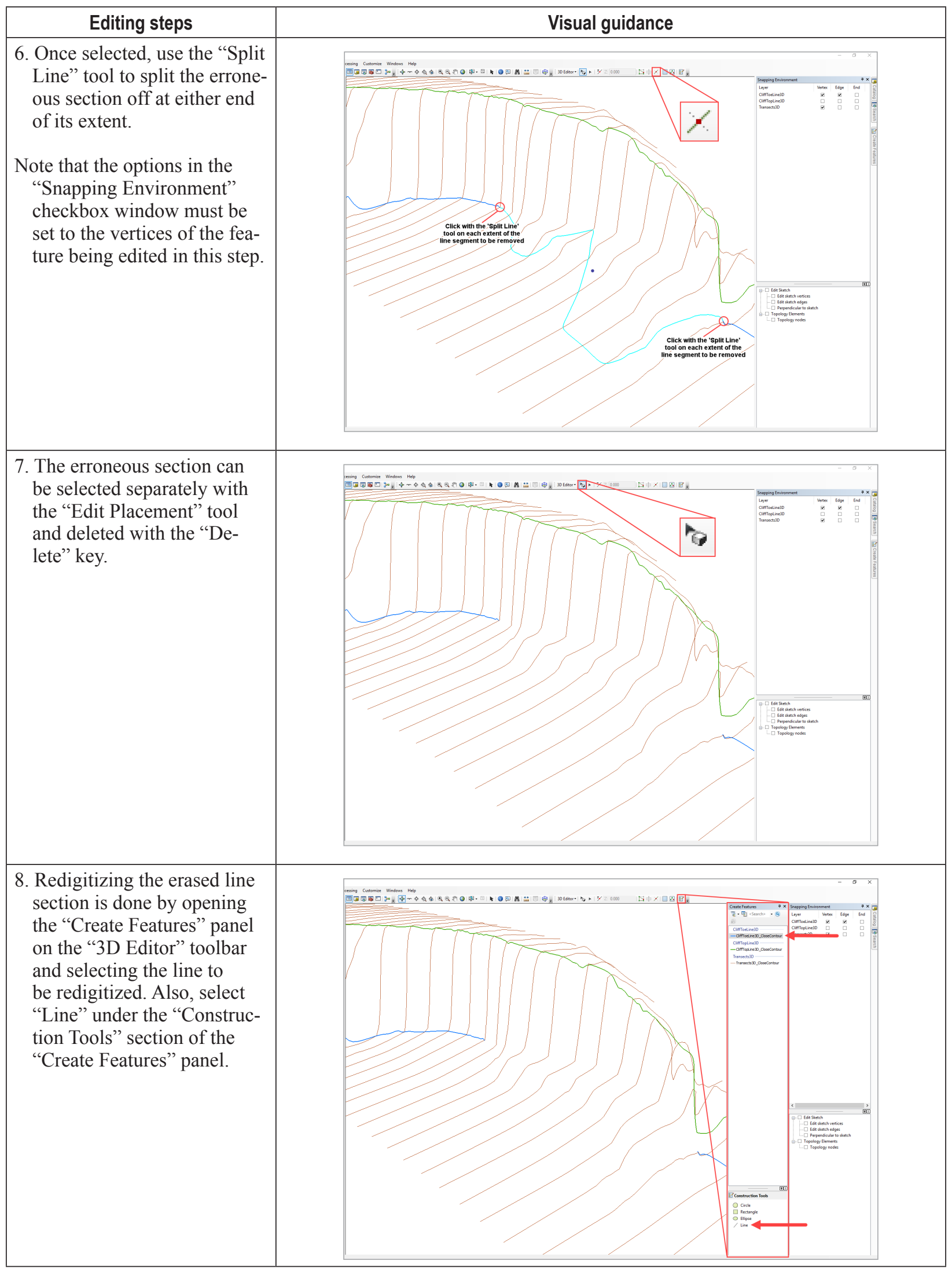

Figure 20. Instructions for editing any cliff feature line in ArcScene.-Continued 


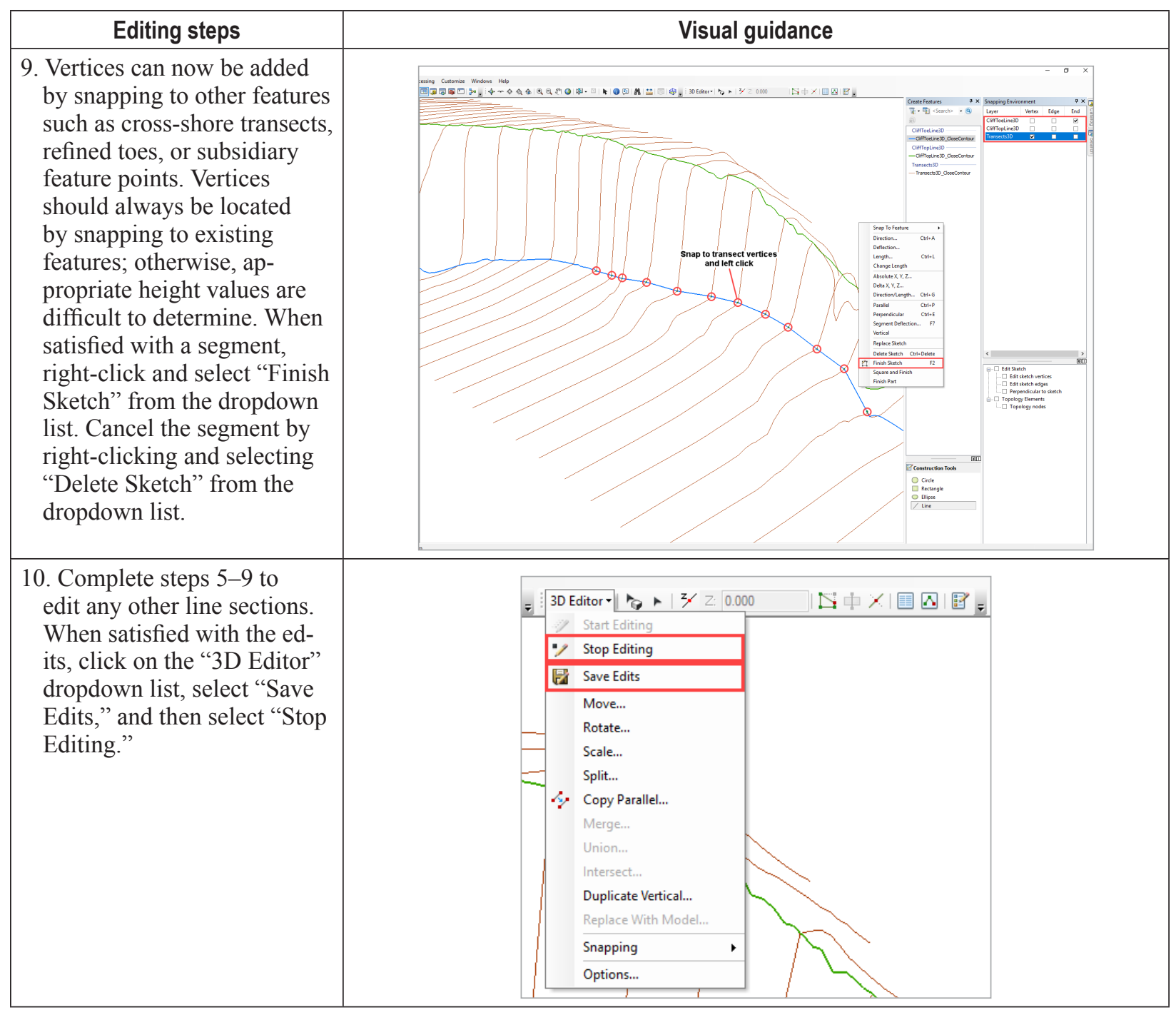

Figure 20. Instructions for editing any cliff feature line in ArcScene.-Continued

TIP: Quickly switch between the "Create Features" and "Snapping Environments" panels by dragging each panel to a different location in the ArcScene window to display both simultaneously.

\subsection{Visualizing Features in ArcMap}

Cliff feature outputs can be added to ArcMap by dragging them from the catalog to the main map panel in the same manner as is done in section 7.1, although it is not recommended to conduct edits in this format because the view is $2 \mathrm{D}$, and the position of delineated features on the cliff face appears ambiguous. During a data review, ArcMap is suitable for generating traditional elevation profile graphs and reviewing any cutback transects generated during processing. 
Complete the steps shown in figure 21 to generate elevation profile graphs and feature point locations across a given cross-shore transect in ArcMap.

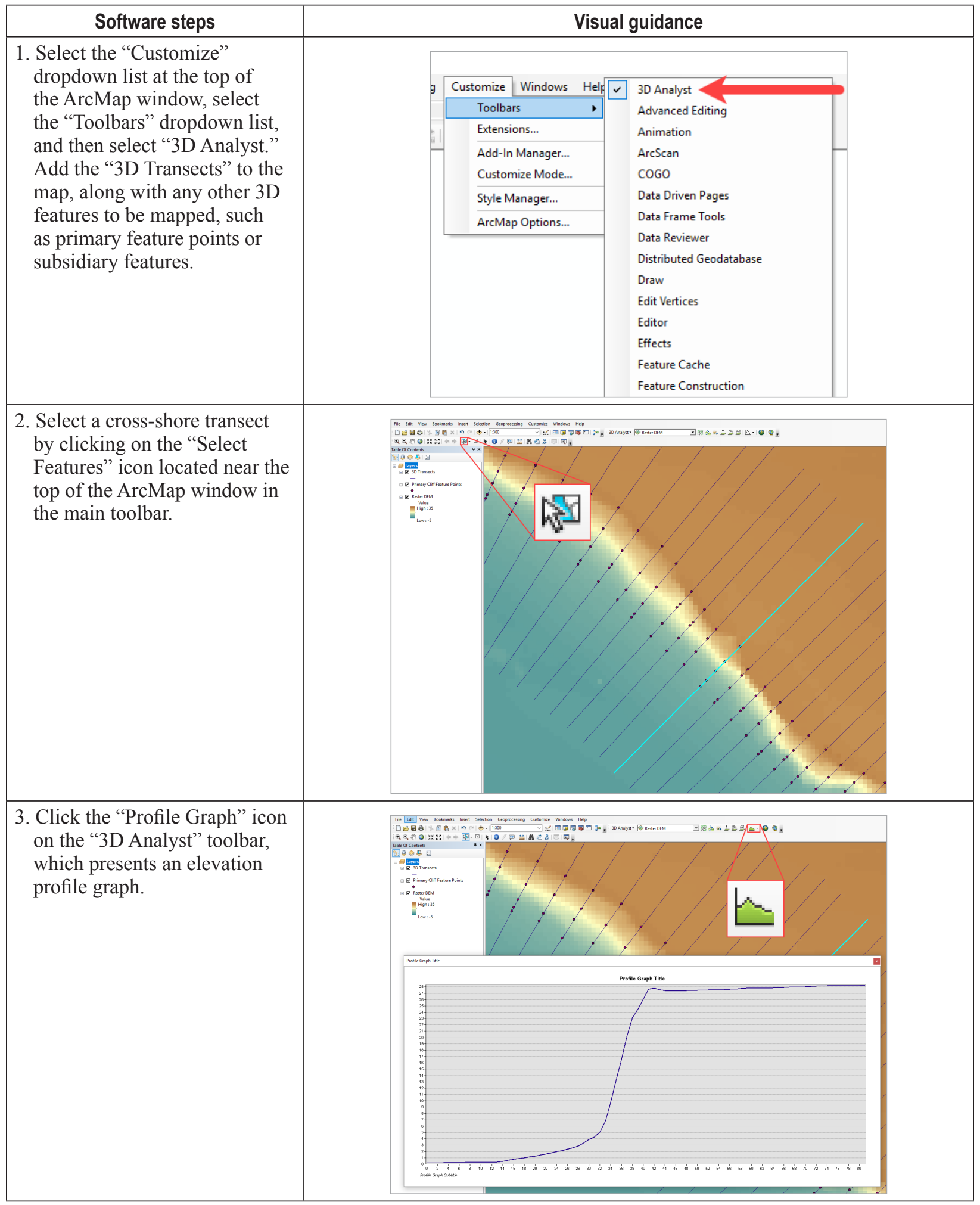

Figure 21. Instructions for graphing cross-shore transects and cliff feature points using ArcGIS Desktop. 


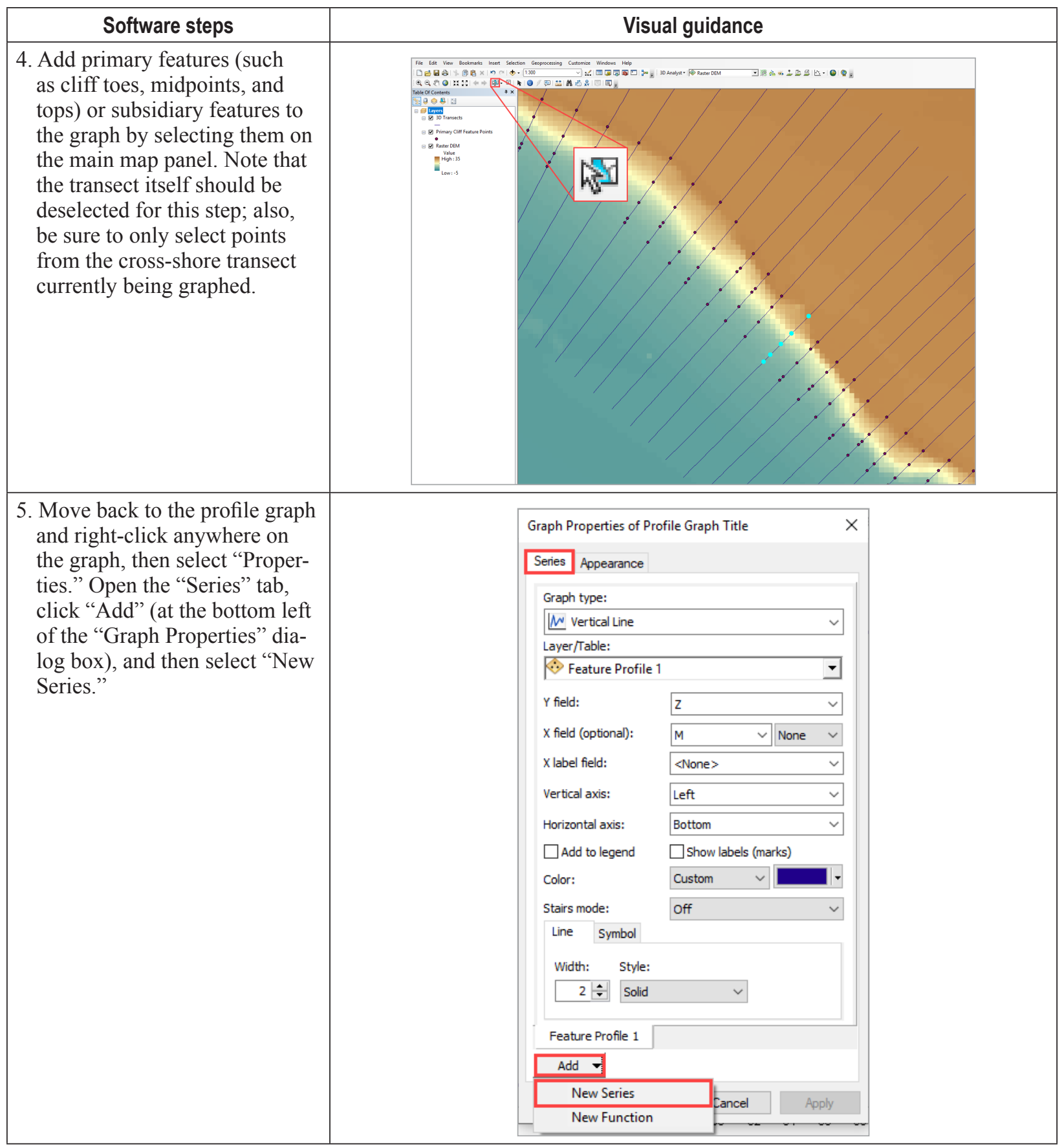

Figure 21. Instructions for graphing cross-shore transects and cliff feature points using ArcGIS Desktop.-Continued 


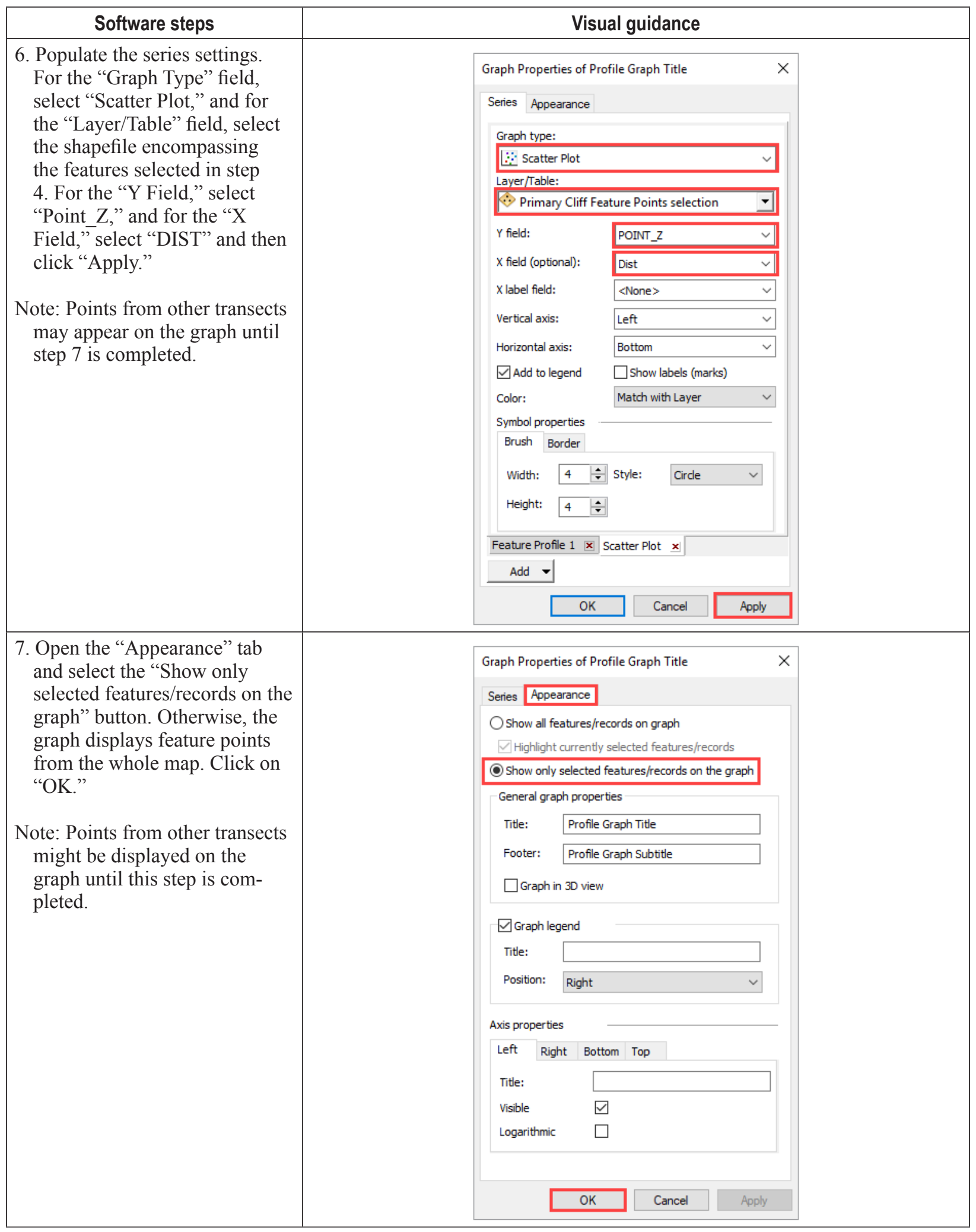

Figure 21. Instructions for graphing cross-shore transects and cliff feature points using ArcGIS Desktop.-Continued 


\begin{tabular}{|c|c|c|c|}
\hline Software steps & \multicolumn{3}{|c|}{ Visual guidance } \\
\hline $\begin{array}{l}\text { 8. The feature points appear on } \\
\text { the elevation profile. Right- } \\
\text { clicking on a point or part of an } \\
\text { elevation profile and selecting } \\
\text { "Identify" displays the eleva- } \\
\text { tion (Point_Z) and distance } \\
\text { (DIST) along the cross-shore } \\
\text { transect at that location, as well } \\
\text { as the type of point ("F_Type," } \\
\text { primary features only). For } \\
\text { instance, "ToeFC" indicates } \\
\text { a toe identified with the first } \\
\text { concavity method, while "Toe" } \\
\text { refers to the basic method. }\end{array}$ & 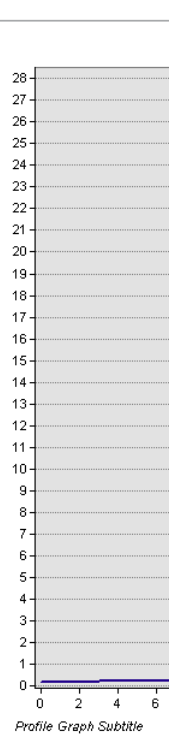 & 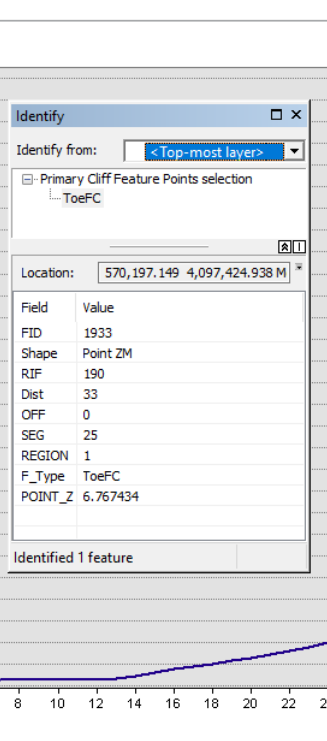 & 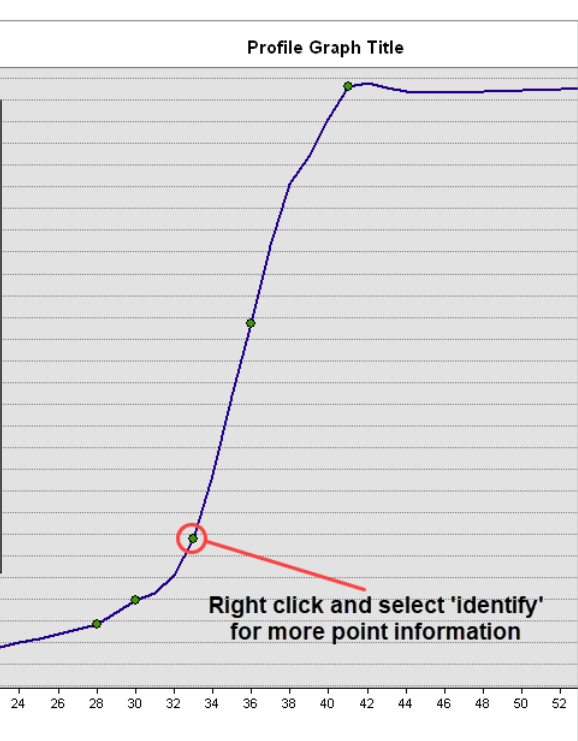 \\
\hline
\end{tabular}

Figure 21. Instructions for graphing cross-shore transects and cliff feature points using ArcGIS Desktop.-Continued

TIP: Change the title and axis labels by navigating to the "Appearance" tab of the graph properties. The elevation profile or point colors can be changed under the "Series" tab. The axis scale and other settings can be changed by right-clicking on the graph and selecting "Advanced Properties."

The "3D Transects" shapefile contains additional information in its attribute table, which can be accessed by right-clicking on its entry in the "Table of Contents" and selecting "Open Attribute Table." Up to five columns (Top_X, Mid_X, Toe_X, ToeHP_X, and ToeFC_X) record the distance along the cross-shore transect where the cliff top, midpoint, basic toe, high-pass toe, and first concavity toe were identified, respectively. The "Shape_Leng" column records the total cross-shore transect length in meters. Two columns, "Start_Elev" and "End_Elev", record the elevations at the start and end of the transect and can be used to calculate the slope of the chord line. The "FMF" column serves two purposes as the unique identifier for the transect and for recording the distance along the offshore baseline at which it was generated. The "FMF" value can be referenced in the "SubsidiaryFeatures3D" and "PrimaryFeaturePoints" attribute table under the "RIF" column, and it also appears in the attribute tables of many intermediate outputs. Users can thereby link the data in any given output to a given cross-shore transect. Both subsidiary and primary feature point shapefiles have "Point_Z" and "DIST" fields, which record the elevation and distance of the point along a given cross-shore transect.

\subsection{Exporting Features to Text Tables}

For point shapefiles such as "PrimaryFeaturePoints" and "SubsidiaryFeatures3D," there are several methods of exporting the results as text tables for use in other software programs. If the user has ArcMap's spatial statistics license enabled, the best method is to select the desired 
point shapefile as an input into the "Export Feature Attribute to ASCII" tool, which exports the $x y$ coordinates of each point, along with all attribute-table information. If the user lacks the spatial statistics license, they can instead run the "Feature Class Z to ASCII" tool, which exports the $x y z$ coordinates of each point feature but omits other attribute-table information. Alternatively, the user can input the desired point shapefile into the "Add XY Coordinates" tool, which appends $x y$ coordinates to the points' attribute table entries and then exports the table as a Microsoft Excel file using the "Table to Excel" tool. This method retains all the attribute table information for the feature.

\subsection{Error Logs}

The CFDT recursively checks outputs for feature line cutbacks and maintains a record of transects in the study area where feature delineation was impossible. To display cross-shore transects where feature line cutbacks were detected, navigate to the "Data" folder and drag the "CutbackTransects" shapefile with the appropriate series name into the main map panel (fig. 12). The "Delete Cutbacks" CFDT option in section 5.2.13 automatically removes cliff feature cutbacks, along with any other section of cliff feature line that comes into the vicinity of a flagged cutback transect. If no transect features are displayed on the map when adding the "CutbackTransects" shapefile, no feature line cutbacks were detected during processing.

Additionally, the "Tables" folder in the working directory contains a "TransectErrorRecords" comma-separated values file (.csv) listing any other transects where errors were encountered during feature delineation. The file can be opened in Microsoft Excel or any text editor, or it can be added to the main map panel. View the entries in ArcMap by right-clicking on the file in the "Table of Contents" and selecting "Open." For this table, the "RIF" column corresponds to a respective cross-shore transect (the "FMF" column on the "3D Transects" attribute table). A short explanation of the problem encountered is provided in the "ErrorType" column. The "ErrorCode" column is for internal use by the tool script.

\subsection{Intermediate Outputs}

Some intermediate outputs generated during processing are kept in the "Scratch," "Scratch.gdb," and "Tables" folders in the CFDT working directory after each processing run. These outputs are useful during troubleshooting; when errors or unusual outputs are recognized, a user can add intermediate outputs to the mapping panel and review their attribute tables, which can provide clues regarding when and why anomalies were generated. The file names are also directly cross-referenced in the tool script, so a user can open the script file and determine which processes an intermediate output was associated with. Intermediate outputs are not appended with a series name, therefore they are overwritten during each subsequent processing run.

\section{Acknowledgments}

Thanks go to the GIS and Python Knowledge Base Help Group at the USGS, including Shane Wright, Marc Hunter, Gregory Cocks, and Curtis Price. This group offered critical debugging advice for aspects of the CFDT and Baseline Builder Tool. Thanks also go to Kyle Glaws, who supported the development of cliff feature delineation at the USGS with an earlier, semiautomated tool that used related algorithmic logic. 


\section{References Cited}

Bird, E.C.F., 1994, Cliff hazards and coastal management: Journal of Coastal Research, special issue no. 12, chap. 21, p. 299-309. [Also available at https://www.jstor.org/stable/25735606.]

Briese, C., 2004, Three-dimensional modelling of breaklines from airborne laser scanner data, in Proceedings, Geo-Imagery Bridging Continents, XXth International Congress for Photogrammetry and Remote Sensing, Istanbul, Turkey, 12-23 July 2004: International Archives of Photogrammetry, Remote Sensing and Spatial Information Sciences, XX ISPRS Congress, commission III, v. XXXV-B3, p. 1097-1102, accessed August 23, 2019, at https://www.isprs.org/proceedings/XXXV/congress/comm3/papers/430.pdf.

Di, K., Wang, J., Ma R., and Li, R., 2003, Automatic shoreline extraction from high-resolution IKONOS satellite imagery, in Proceedings, ASPRS Annual Conference, 2003, Anchorage, Alaska-Technology-Converging at the Top of the World, May 5-9: Bethesda, Md., American Society for Photogrammetry and Remote Sensing, 12 p. [Also available at: https://www.researchgate.net/publication/241058589_Automatic_shoreline_extraction_from_ high_resolution_IKONOS_satellite_imagery.]

Emery, K.O., and Kuhn, G.G., 1982, Seacliffs_-Their processes, profiles, and classification: Geological Society of America Bulletin, v. 93, July, p. 644-654, accessed May 05, 2019, at https://doi.org/10.1130/0016-7606(1982)93\%3C644:SCTPPA\%3E2.0.CO;2.

Griggs, G.B., and Patsch, K., 2004, California's coastal cliffs and bluffs, in Hampton, M.A., and Griggs, G.B., eds., Formation, evolution, and stability of coastal cliffs-Status and trends: U.S. Geological Survey Professional Paper 1693, p. 53-64, accessed May 05, 2019, at https://doi.org/10.3133/pp1693.

Hapke, C., and Plant, N., 2010, Predicting coastal cliff erosion using a Bayesian probabilistic model: Marine Geology, v. 278, nos. 1-4, p. 140-149. [Also available at: https://doi.org/10.1016/j.margeo.2010.10.001.]

Hapke, C.J., Limber, P., and Glaws, K., 2018, Automated 4D coastal slope failure characterization tool for assessment of coastal vulnerability [abs.] in AGU 100, Fall Meeting 2018, Washington, D.C., 10-14 December: [Washington, D.C.], American Geophysical Union, abstract no. EP13A-05, accessed July 25, 2019, at https://ui.adsabs.harvard.edu/ abs/2018AGUFMEP13A..05H/abstract.

Hapke, C.J., and Reid, D., 2007, The National assessment of shoreline change, part 4-Historical coastal cliff retreat along the California coast: U.S. Geological Survey Open-File Report 2007-1133, 51 p., accessed April 25, 2019, at https://doi.org/10.3133/ofr20071133.

Himmelstoss, E.A., Henderson, R.E., Kratzmann, M.G., and Farris, A.S., 2018, Digital Shoreline Analysis System (DSAS) version 5.0 user guide: U.S. Geological Survey Open-File Report 2018-1179, 110 p., accessed July 7, 2019, at https://doi.org/10.3133/ofr20181179.

Palaseanu-Lovejoy, M., Danielson, J., Thatcher, C., Foxgrover, A., Barnard, P., Brock, J., and Young, A., 2016, Automatic delineation of seacliff limits using lidar-derived high-resolution DEMs in southern California: Journal of Coastal Research, special issue no. 76, p. 162-173. [Also available at https://doi.org/10.2112/SI76-014.]

Richter, A.; Faust, D., and Maas, H.-G., 2013, Dune cliff erosion and beach width change at the northern and southern spits of Sylt detected with multi-temporal Lidar: Catena, v. 103, April, p. 103-111, accessed June 12, 2019, at https://doi.org/10.1016/j.catena.2011.02.007. 
Ruggiero, P., Kratzmann, M.G., Himmelstoss, E.A., Reid, D., Allan, J., and Kaminsky, G., 2013, National assessment of shoreline change-Historical shoreline change along the Pacific Northwest coast: U.S. Geological Survey Open-File Report 2012-1007, 62p. [Also available at https://doi.org/10.3133/ofr20121007.]

Rutzinger, M., Höfle, B., and Kringer, K., 2012, Accuracy of automatically extracted geomorphological breaklines from airborne LiDAR curvature images: Geografiska Annaler-Series A, Physical Geography, v. 94, no. 1, p. 33-42, accessed April 17, 2019, at https://doi.org/10.1111/j.1468-0459.2012.00453.x.

Warrick J.A., Ritchie A.C., Adelman, G., Adelmann, K., and Limber, P.W., 2017, New techniques to measure cliff change from historical oblique photographs and structure-from-motion photogrammetry: Journal of Coastal Research, v. 33, no. 1, p.39-55. [Also available at https://doi.org/10.2112/JCOASTRES-D-16-00095.1.]

Warrick, J.A., Ritchie, A.C., Schmidt, K.M., Reid, M.E., and Logan, J., 2019, Characterizing the catastrophic 2017 Mud Creek landslide, California, using repeat structure-from-motion (SfM) photogrammetry: Landslides, v. 16, no. 6, p. 1201-1219, accessed June 2, 2019, at https://doi.org/10.1007/s10346-019-01160-4. 


\section{Glossary}

Baseline Builder Tool A software tool that automatically generates offshore baselines adjacent to the seacliffs represented in a DEM, satisfying a required input for the Cliff Feature Delineation Tool that would otherwise necessitate manual digitization.

Baseline Distance A Baseline Builder Tool input representing the desired seaward distance, in meters, between the cliff face and the offshore baseline.

Baseline Smoothing Tolerance An optional parameter in the Baseline Builder Tool that specifies the extent of smoothing performed on a baseline before it is processed by the CFDT.

Basic Delineation A cliff delineation mode of the CFDT that uses the vertical distance from the primary chord line to identify cliff top and toe features.

Cliff Feature Delineation Tool (CFDT) Software designed to speed the spatial delineation of cliff morphology while removing the potential for variable human interpretation, ultimately making outputs more repeatable.

Cliff Height The minimum height, in meters, of cliffs that are to be classified in the study area using the Baseline Builder Tool.

Delete Cutbacks An error-handling option in the CFDT that deletes any cliff feature lines that cut back on themselves.

Delineation Mode An input that determines which and how many algorithms in the CFDT are used to identify final cliff features.

Filter Type A control that allows the selection of one of the two elevation filtering types that can be used to generate a baseline in the Baseline Builder Tool: "Automatic" or "Elevation Threshold."

First Concavity A refinement method in the CFDT that identifies a cliff toe by projecting a chord line between the coarsely delineated toe and the cliff midline and then running a one-dimensional slope-analysis kernel across the elevation profile surface between the toe and midline.

Flag Cutbacks An error-handling option in the CFDT that flags regions where cliff feature lines cut back on themselves rather than simply deleting the features.

High Pass A refinement method in the CFDT that identifies the cliff toe by analyzing a highpass profile of the beach and cliff.

High Pass Threshold A control in the CFDT that allows a user to tune cliff toe delineation during the high-pass refinement method.

Input DEM The DEM input into the Baseline Builder Tool containing the cliff features from which the baseline should be built seaward; it is the same raster DEM that is to be used for cliff feature delineation in the CFDT.

Kernel Size An input that modifies the size of the one-dimensional analysis kernel used in "First Concavity" toe refinement and "Subsidiary Feature Delineation."

Landward Distance A CFDT input that indicates the distance in meters that the cross-shore transect projects landward from the offshore baseline.

Maximum Elevation Threshold A filter setting in the Baseline Builder Tool that causes polylines occurring above a given elevation to be filtered out of baseline results under the "Manual Threshold" filter-type setting and is unavailable under the "Automatic" filter-type setting. 
Minimum Elevation Threshold A filter setting in the Baseline Builder Tool that causes polylines occurring below a given elevation to be filtered out of baseline results under the "Manual Threshold" filter-type setting and is unavailable under the "Automatic" filter-type setting.

Multi Delineation A delineation mode in the CFDT that executes all 3 delineation methods for the cliff toe.

Neighborhood The size, in meters, of the 2D kernel size used in the Baseline Builder Tool to scan the DEM for the user-specified "Cliff Height."

Offshore Baseline A polyline generated by the Baseline Builder Tool, or manually digitized, that runs alongshore and seaward of the seacliff features to be delineated. This polyline is a prerequisite for running the CFDT.

Output Cleanup An input in the CFDT that determines how to handle feature line cutbacks or regions where more than one cliff top-toe instance is identified along a single cross-section profile.

Output Name The file name suffix given to the output digitization guide or automatic baseline. All outputs use the name format "AutoBase_OutputName.shp".

Output Smoothing Tolerance An input into the CFDT measured in meters that determines the level of smoothing performed on output cliff feature lines.

Refined Delineation Outputs cliff tops and midpoints as with "Basic Delineation," but cliff toes are derived from a selected "Refined Delineation" method.

Refinement Method One of two options available for toe-position refinement in the CFDT: "High Pass" or "First Concavity."

Seaward Distance ACFDT input that indicates the distance in meters that the cross-shore transect projects seaward from the offshore baseline.

Series Name A CFDT input that adds a user-specified suffix to the names of final model outputs for the purpose of identifying tool results from one run to the next.

Smooth Baseline An input that determines whether the CFDT smooths the input baseline before it proceeds with cliff feature identification.

Smoothing Tolerance A Baseline Builder Tool input, in meters, that controls the smoothness of the curvature for output baselines.

Subsidiary Feature Delineation A selectable CFDT mode that identifies convexities and concavities across the cliff face other than the cliff top and toe using a one-dimensional analysis kernel.

Transect Interval An input in the CFDT that defines the distance in meters, along the offshore baseline, between generated transects. 

